\title{
The two metallicity groups of the globular cluster M 22: a chemical perspective ${ }^{\star, \star \star}$
}

\author{
A. F. Marino ${ }^{1,2}$, C. Sneden ${ }^{3}$, R. P. Kraft ${ }^{4}$, G. Wallerstein ${ }^{5}$, J. E. Norris ${ }^{6}$, G. Da Costa ${ }^{6}$, A. P. Milone ${ }^{7,8}$, I. I. Ivans ${ }^{9}$,
} G. Gonzalez ${ }^{5,10}$, J. P. Fulbright ${ }^{4,11}$, M. Hilker ${ }^{12}$, G. Piotto ${ }^{2}$, M. Zoccali ${ }^{13}$, and P. B. Stetson ${ }^{14}$

${ }^{1}$ Max-Planck-Institut für Astrophysik, Karl-Schwarzschild-Str. 1, 85741 Garching bei München, Germany e-mail: amarino@MPA-Garching.MPG.DE

2 Dipartimento di Astronomia, Università di Padova, Vicolo dell'Osservatorio 3, 35122 Padova, Italy e-mail: [anna.marino;giampaolo.piotto]@unipd.it

3 Department of Astronomy and McDonald Observatory, The University of Texas, Austin, TX 78712, USA e-mail: chris@verdi.as.utexas.edu

${ }^{4}$ UCO/Lick Observatory, Department of Astronomy and Astrophysics, University of California, Santa Cruz, CA 95064, USA e-mail: kraft@ucolick.org

5 Department of Astronomy, University of Washington, Seattle, WA 98195, USA e-mail: wall@astro.washington. edu

${ }^{6}$ Research School of Astronomy and Astrophysics, Australian National University, Cotter Road, Weston, ACT 2611, Australia e-mail: [jen;gdc]@mso.anu.edu.au

7 Instituto de Astrofísica de Canarias, La Laguna, Tenerife, Spain e-mail: milone@iac.es

8 Departamento de Astrofísica, Universidad de La Laguna, Tenerife, Spain

9 Department of Physics and Astronomy, The University of Utah, Salt Lake City, UT 84112, USA e-mail: iii@physics.utah.edu

10 Physics Department, Grove City College, 100 Campus Drive, Grove City, 16127 Pennsylvania, USA e-mail: gonzalezg@gcc.edu

11 Department of Physics and Astronomy, Johns Hopkins University, Baltimore, MD 21218, USA e-mail: jfulb@skysrv.pha.jhu.edu

12 European Southern Observatory, Karl-Schwarzschild-Str. 2, 85748 Garching bei München, Germany e-mail: mhilker@eso.org

13 Pontificia Universidad Católica de Chile, Departmento de Astronomía y Astrofisíca, Casilla 306, Santiago 22, Chile e-mail: mzoccali@astro.puc.cl

14 Herzberg Institute of Astrophysics, National Research Council Canada, 5071 West Saanich Road, Victoria, BC V9E 2E7, Canada e-mail: Peter.Stetson@nrc-cnrc.gc.ca

Received 19 January 2011 / Accepted 26 April 2011

\section{ABSTRACT}

\begin{abstract}
We present a detailed chemical composition analysis of 35 red giant stars in the globular cluster M 22. High resolution spectra for this study were obtained at five observatories, and analyzed in a uniform manner. We have determined abundances of representative light proton-capture, $\alpha$, Fe-peak and neutron-capture element groups. Our aim is to better understand the peculiar chemical enrichment history of this cluster, in which two stellar groups are characterized by a different content in iron, neutron capture elements $\mathrm{Y}, \mathrm{Zr}$ and $\mathrm{Ba}$, and $\alpha$ element $\mathrm{Ca}$. The principal results of this study are: (i) substantial star-to-star metallicity scatter $(-2.0 \lesssim[\mathrm{Fe} / \mathrm{H}] \lesssim-1.6)$; (ii) enhancement of $s$-process/ $r$-process neutron-capture abundance ratios in a fraction of giants, positively correlated with metallicity; (iii) sharp separation between the $s$-process-rich and $s$-process-poor groups by $[\mathrm{La} / \mathrm{Eu}]$ ratio; (iv) possible increase of $[\mathrm{Cu} / \mathrm{Fe}] \mathrm{ratios}$ with increasing $[\mathrm{Fe} / \mathrm{H}]$, suggesting that this element also has a significant $s$-process component; and $(v)$ presence of $\mathrm{Na}-\mathrm{O}$ and $\mathrm{C}-\mathrm{N}$ anticorrelations in both the stellar groups.
\end{abstract}

Key words. techniques: spectroscopic - stars: abundances - stars: Population II - globular clusters: individual: M 22 (NGC 6656)

* Based on data collected at: Anglo-Australian Telescope with the University College London Echelle Spectrograph, Apache Point Observatory with the ARC Echelle Spectrograph, Lick Shane $3.0 \mathrm{~m}$ Telescope with the Hamilton Echelle Spectrograph, McDonald Smith $2.7 \mathrm{~m}$ Telescope with the Robert G. Tull Coudé Spectrograph, and European Southern Observatory with the FLAMES/UVES spectrograph.

$\star \star$ Tables 3-6 are available in electronic form at http://www. aanda.org

\section{Introduction}

In recent years, a large amount of observational evidence, both from high resolution spectroscopy and from photometry, has established that globular clusters (GC) can host more than one stellar population. Photometric evidence of multiple stellar populations has been recently observed in many GCs in the form of multiple main sequences (Bedin et al. 2004; Piotto et al. 2007; Anderson et al. 2009; and Milone et al. 2010), split sub-giant branches (Milone et al. 2008; and Piotto 2009), and multiple 
red-giant branches (e.g. Marino et al. 2008; Lee et al. 2009; Lind et al. 2011a).

Spectroscopic clues, often easy to detect simply by inspection of high-resolution spectra, arise in derived elemental abundance variations. Most GCs are mono-metallic (Carretta et al. 2009a), i.e. they have no detectable dispersion in $[\mathrm{Fe} / \mathrm{H}]^{1}$. But all clusters studied to date with substantial stellar samples exhibit star-to-star variations in the light elements $\mathrm{C}, \mathrm{N}, \mathrm{O}, \mathrm{Na}$, $\mathrm{Mg}$, and $\mathrm{Al}$. These variations do not occur randomly, but have obvious correlations and anticorrelations, which clearly point to the actions of high-temperature proton fusion cycles that have processed $\mathrm{C}$ and $\mathrm{O}$ into $\mathrm{N}$, (unseen) $\mathrm{Ne}$ to $\mathrm{Na}$, and $\mathrm{Mg}$ to $\mathrm{Al}$.

Proton-capture reactions to effect $\mathrm{Ne} \rightarrow \mathrm{Na}$ conversion require fusion-region temperatures $T \gtrsim 40 \times 10^{6} \mathrm{~K}$, and $\mathrm{Mg} \rightarrow \mathrm{Al}$ conversion can only occur with even higher temperatures. Such conditions cannot be achieved in the H-fusion layers of the presently observed low-mass $\left(\simeq 0.8 M_{\odot}\right)$ GC stars. This suggests that the stars observed now were formed from cluster material that had been polluted in proton-capture products by a prior generation of cluster stars. Today, it is widely accepted that the observed variations in light elements provide strong evidence for the presence of different generations of stars in GCs, with the younger stars born in a medium enriched in the material ejected by earlier generation stars. However the debate on the possible polluters is still open (Ventura et al. 2001; D'Antona \& Ventura 2007; Decressin et al. 2007).

The mono-metallicity of most GCs has excluded supernovae from being responsible for the chemical enrichment of the intracluster medium from which the second generation formed, because supernovae ejecta would be enriched in Fe-peak elements. We know of some exceptions, most notably $\omega$ Centauri. This most massive cluster is an anomaly among GCs because its $\mathrm{Fe}$ variations are huge, spanning more than 1 dex. However, there have been long-held suspicions that M 22 has metallicity variations. A large number of photometric and spectroscopic studies have attempted to verify or disprove this idea. For example, Hesser et al. (1977) and Peterson \& Cudworth (1994) showed a significant spread along the RGB in M 22 in observed $(B-V)$ and Strömgren colours. Norris \& Freeman (1983) used lowresolution spectra of about 100 red giants (RGB) to demonstrate the existence of star-to-star variations in the strengths of the $\mathrm{Ca}$ II $\mathrm{H} \& \mathrm{~K}$ lines, which they interpreted as a $\sim 0.30$ dex spread in $\mathrm{Ca}$ abundances.

However, M22 lies nearly in the Milky Way plane, toward the Bulge $\left(\left[(l, b) \sim\left(10^{\circ}, 7^{\circ}\right)\right]\right)$. As such, it suffers significant dust extinction, with probable reddening variations across the face of the cluster. This has limited conclusions that could be drawn from the photometric and spectroscopic variations present in M 22. Early spectroscopic studies claiming a spread in metallicity, with $-1.4<[\mathrm{Fe} / \mathrm{H}]<-1.9$, included those of Pilachowski et al. (1982), based on 6 stars, and Lehnert et al. (1991), based on 4 stars. On the other hand, neither Cohen (1981), nor Gratton (1982) found a significant M 22 metallicity variation within the three stars that each analyzed. These early studies are not necessarily in contradiction because they all are conclusions from small sample sizes.

Recently, intrinsic Fe variations in M22 have been confirmed definitively by Marino et al. (2009, hereafter M09) and Da Costa et al. (2009, hereafter DC09). In particular M09, from

\footnotetext{
1 We adopt standard stellar abundance notation (Helfer et al. 1959): for elements $\mathrm{A}$ and $\mathrm{B},[\mathrm{A} / \mathrm{B}] \equiv \log _{10}\left(N_{\mathrm{A}} / N_{\mathrm{B}}\right)_{\star}-\log _{10}\left(N_{\mathrm{A}} / N_{\mathrm{B}}\right)_{\odot}$. We also define $\log \epsilon(\mathrm{A}) \equiv \log _{10}\left(N_{\mathrm{A}} / N_{\mathrm{H}}\right)+12.0$, and and use $[\mathrm{Fe} / \mathrm{H}]$ synonymously with stellar overall metallicity.
}

the analysis of high resolution UVES spectra of 17 stars, found that M 22 shows a complex chemical pattern that resembles the extreme case of $\omega$ Cen (see also Da Costa \& Marino 2010). Stars in M 22 show intrinsic variations in Fe, albeit significantly smaller than the ones observed in $\omega$ Cen, i.e. while the difference between the mean Fe abundances of different groups of stars is 0.14 dex (M09), stars in $\omega$ Cen span a range of $\sim 1.5$ dex. Additionally, M09 argued for the presence of two different stellar populations in this cluster characterized by significant differences in neutron-capture (n-capture) elements Y, Ba, and $\mathrm{Zr}$. Light proton-capture elements vary in M 22 as they do in most clusters as described above, but their abundance variations are uncorrelated to those of the $n$-capture elements. The two M 22 populations also appear to have different $[\mathrm{Ca} / \mathrm{Fe}]$ ratios, but no linkage to the proton-capture elements is evident.

The multiple populations of M22 are now clearly manifest in a photometric split in the sub-giant branch (SGB) region revealed in Hubble Space Telescope images (M09; Piotto 2009). The split SGB points towards the presence of two stellar generations, which are likely related to chemical composition differences. However, M09 argued that evolutionary models cannot entirely reproduce the size of the split by considering only the metallicity spread (but see also DC09). Probably the origin of the split is more complex, and could involve also variations in the total CNO abundance, as proposed by Cassisi et al. (2008) and Ventura et al. (2009) for NGC 1851, in contrast to the usual assumption of constant $\mathrm{C}+\mathrm{N}+\mathrm{O}$ inside stars in a given $\mathrm{GC}$.

Since the 1980's we have known that large CO band strength variations (Frogel et al. 1983) are present among M22 stars, and these are accompanied by often strong $\mathrm{CN}$ enhancements (Cohen 1981; Norris \& Freeman 1983). More recently, Kayser et al. (2008) confirmed the presence of both $\mathrm{CN}$-weak and $\mathrm{CN}$ strong stars in M 22, with the majority of stars being $\mathrm{CN}$-weak group. Their pager found no evidence for a $\mathrm{CN}-\mathrm{CH}$ anticorrelation. Indeed, Norris \& Freeman had noted that in M 22, contrary to normal GCs, CN strengths are positively correlated with those of $\mathrm{CH}$. They also found that $\mathrm{CH}$ and $\mathrm{CN}$ are correlated with $\mathrm{Ca}$, with the correlation being tighter between $\mathrm{CH}$ and $\mathrm{Ca}$. This suggests that a common source is responsible for the $\mathrm{C}$ and $\mathrm{Ca}$ enhancements, and a different mechanism is responsible for the $\mathrm{N}$ enhancements. This further argues that a simple conversion of $\mathrm{C}$ to $\mathrm{N}$ by $\mathrm{CN}$ cycling cannot be the unique cause of the spread in $\mathrm{CN}$ index. Brown et al. (1990) studied CNO abundances in seven M 22 RGB stars, and found evidence in two of the stars for a large overabundance of $\mathrm{N}$, corresponding to a higher $\mathrm{CNO}$ total abundance. This behavior cannot be explained with enrichment from material that simply has undergone the complete CNO cycle.

The various chemical anomalies of M 22 stars indicate that this GC has undergone a complex, and still unclear, chemical enrichment history. Similarly to $\omega \mathrm{Cen}, \mathrm{Fe}$ and $\mathrm{Ca}$ variations suggest that core-collapse supernovae have had a role in the pollution of the intra-cluster medium from which the present generation of slightly Fe-enriched stars formed. At the same time, the presence of two different groups in some $n$-capture elements suggests that at the same epoch a number of low mass AGB stars ( $\sim M_{\odot}$, Ventura et al. 2009) experienced the third dredge-up and polluted the intra-cluster medium with $s$-process and triple $\alpha$ products.

In this study we present a new high-resolution spectroscopic analysis of a larger sample of RGB stars in M 22 than has been conducted to date. Altogether our sample now consists of 35 stars. Our aim is to study the chemical properties of different stellar populations in this cluster in order to re-construct its 
Table 1. Sources of the spectroscopic data.

\begin{tabular}{|c|c|c|c|c|c|c|}
\hline Instrument $^{a}$ & $R^{b}$ & $\lambda$ range & $\begin{array}{c}S / N \\
@ 5000 \AA\end{array}$ & $\begin{array}{c}S / N \\
@ 7000 \AA\end{array}$ & \# stars & Reference \\
\hline AAT-UCLES & 38000 & $5050-7300$ & 50 & 100 & 10 & $\begin{array}{c}\text { Diego et al. (1990) } \\
\text { Norris et al. (1995b) }\end{array}$ \\
\hline APO-ARCES & 37500 & $4000-9000$ & 50 & 75 & 5 & Wang et al. (2003) \\
\hline MCD-2DC & 60000 & $4200-9000$ & 70 & 120 & 6 & Tull et al. (1995) \\
\hline LICK-HAM & 50000 & $4800-8800$ & 50 & 90 & 9 & $\begin{array}{l}\text { Vogt et al. (1987) } \\
\text { Valenti et al. (1995) }\end{array}$ \\
\hline VLT-UVES & 45000 & $\begin{array}{l}3300-4500 \\
4800-6800 \\
\end{array}$ & 100 & 120 & 16 & $\begin{array}{l}\text { Dekker et al. (2000) } \\
\text { Marino et al. (2009) }\end{array}$ \\
\hline
\end{tabular}

Notes. ${ }^{(a)}$ Short-hand notation adopted in this paper. ${ }^{(b)} R \equiv \lambda / \Delta \lambda$.

chemical enrichment history. The layout of this paper is as follows: Sect. 2 is a brief overview of the data set; Sects. 3 and 4 contain descriptions of model atmosphere parameter and abundance derivations; Sect. 5 presents the abundance results; Sect. 6 demonstrates the links between our abundances and spectroscopic/photometric indices of M 22 giants; and Sect. 7 summarizes and discusses our findings.

\section{Data sources}

High-resolution spectra of M 22 giants were obtained at five observatories. In Table 1 we summarize the properties of these instruments, giving their resolving powers, approximate spectral range coverage, typical signal-to-noise values per pixel for the reduced spectra, the number of observed stars and references to information on the instruments. Here are some notes about the observations and references to more detailed information.

- Anglo-Australian Telescope, University College London Echelle Spectrograph (hereafter called AAT): observations and reductions were described by Norris et al. (1995b). The reductions were accomplished with a combination of tasks from the IRAF ${ }^{2}$ and FIGARO ${ }^{3}$ software packages. That paper also tabulates their equivalent widths, but we do not use those measurements in this paper; for uniformity in our analyses we re-measured equivalent widths from the original spectra. Comparison of our equivalent widths with those of Norris et al. shows good agreement, with a mean difference of $-1.8 \mathrm{~m} \AA(\sigma=4.5 \mathrm{~m} \AA)$ and no trend with line strength.

- Apache Point Observatory, $3.5 \mathrm{~m}$ telescope, ARC Echelle Spectrograph (hereafter, APO): observation and reduction procedures were identical to those described by Laws \& Gonzalez (2003). In particular, the data were reduced using standard routines in IRAF for flat fielding, wavelength calibration and background subtraction.

- Lick Shane $3.0 \mathrm{~m}$ Telescope, Hamilton Echelle (hereafter, LICK): we followed the procedures outlined in Ivans et al. (1999). The reductions were carried out using the VISTA software package (Goodrich \& Veilleux 1988).

- McDonald Smith 2.7 m Telescope, Robert G. Tull Coudé Spectrograph (hereafter, MCD): observations and reductions were identical to those employed by Ivans et al. (1999) for their high-resolution study of stars in the GC M 4.

- ESO Very Large Telescope, Ultraviolet and Visual Echelle Spectrograph (programmes: 68.D-0332 and 71.D-0217;

\footnotetext{
2 IRAF is distributed by NOAO, which is operated by AURA, under cooperative agreement with the US National Science Foundation.

${ }^{3}$ http://www.aao.gov.au/figaro/
}

hereafter UVES): see M09 for a discussion of the observations and reductions.

Equivalent widths (EWs) were measured by fitting Gaussian profiles to isolated stellar absorption lines. For each line, we selected a region of $10 \AA$ centered on the line itself to estimate continuum placement. This value is a good compromise between having enough points to build reasonable statistics, and avoiding regions where the spectrum is not sufficiently flat. Then we built the histogram of the distribution of the flux where the peak is a rough estimation of the continuum. We refined this determination by fitting a parabolic curve to the peak and using the vertex as our continuum estimation. Finally, the continuum determination was revised by eye and corrected by hand if a clear discrepancy with the spectrum was found. We rejected the EW of a transition if any of the following problems were detected: non-Gaussian line profile; central observed wavelength mismatch with the expected line list wavelength; line breadth either substantially broader or narrower with respect to the mean FWHM. We verified that the Gaussian shape was a good approximation for our spectral lines, so no Lorentzian correction was applied.

In Table 2 we provide a list of basic data for each observed star, together with the instrument source. The different spectroscopic data sets were analyzed separately, but the results are combined in the abundance discussion. The table lists magnitudes and colours as observed (no corrections for dust extinction), and the adopted extinction $A_{V}$ for each star. The broadband $V, B$, and $I$ magnitudes are from Stetson's photometric database $^{4}$ while the $K$ magnitudes are from the 2MASS Point Source Catalog (Skrutskie et al. 2006). The Strömgren colours $b-y$ and $m_{1}$ are taken from Richter et al. (1999).

We computed extinction values $A_{V}$ in the following manner. M 22 has large mean reddening, $E(B-V)=0.34$ (Harris 1996). Additionally, there is evidence for differential reddening at a level of nearly a tenth of a magnitude in $E(B-V)$ across the face of the cluster as evidenced by a spread in the colours and magnitudes of its evolutionary sequence. We corrected the cluster mean $E(B-V)$ for differential reddening with a method that will be described by Milone et al. (2011). This technique is similar to the ones adopted by Sarajedini et al. (2007) and Milone et al. (2009). Briefly, we first defined the fiducial main sequence for the cluster. Then for each star we estimated how much other observed stars in its cluster spatial vicinity systematically lie to the red or the blue of the fiducial sequence. This systematic colour offset is indicative of the local differential reddening. We applied the M 22 mean $E(B-V)$ plus the differential

\footnotetext{
4 Available at http://www3. cadc-ccda.hia-iha.nrc-cnrc.gc . ca/community/STETSON/
} 
Table 2. M 22 targets: instruments, positions, and photometric data.

\begin{tabular}{|c|c|c|c|c|c|c|c|c|c|c|c|}
\hline $\mathrm{ID}^{a}$ & ID(M09) & SOURCE $^{b}$ & $\alpha(2000)$ & $\delta(2000)$ & $B^{c}$ & $V^{c}$ & $I^{c}$ & $K^{d}$ & $b-y^{e}$ & $m_{1}^{e}$ & $A_{V}$ \\
\hline $\mathrm{I}-12$ & 200031 & AAT, MCD,UVES & $18: 36: 27.36$ & $-23: 51: 26.1$ & 13.177 & 11.672 & 10.000 & 7.893 & 0.999 & 0.173 & 0.998 \\
\hline I-27 & 200083 & APO, UVES & $18: 36: 30.58$ & $-23: 52: 48.7$ & 13.662 & 12.393 & 10.903 & 8.952 & 0.480 & -0.001 & 1.017 \\
\hline $\mathrm{I}-36$ & & LICK & $18: 36: 30.07$ & $-23: 53: 37.5$ & 13.342 & 11.931 & 10.320 & 8.337 & 0.961 & 0.120 & 1.024 \\
\hline I-37 & & LICK & $18: 36: 30.02$ & $-23: 53: 50.1$ & 13.450 & 12.008 & 10.372 & 8.279 & 0.969 & 0.148 & 1.025 \\
\hline I-53 & 200101 & UVES & $18: 36: 38.36$ & $-23: 54: 01.3$ & 14.051 & 12.690 & 11.155 & 9.207 & 0.910 & 0.178 & 1.068 \\
\hline I-57 & & AAT, APO & $18: 36: 38.27$ & $-23: 53: 13.0$ & 13.578 & 11.981 & 10.290 & 8.089 & 1.039 & 0.371 & 1.034 \\
\hline I-80 & 88 & UVES & $18: 36: 36.06$ & $-23: 50: 16.1$ & 13.910 & 12.527 & 11.011 & 9.064 & 0.900 & 0.251 & 1.066 \\
\hline I-85 & 200080 & UVES & $18: 36: 32.82$ & $-23: 51: 10.9$ & 13.747 & 12.473 & 10.978 & 9.117 & 0.867 & 0.065 & 1.083 \\
\hline I-86 & 200068 & AAT, UVES & $18: 36: 32.13$ & $-23: 51: 31.4$ & 13.749 & 12.328 & 10.689 & 8.660 & 0.969 & 0.103 & 1.064 \\
\hline I-92 & & LICK & $18: 36: 33.28$ & $-23: 52: 32.5$ & 13.141 & 11.561 & 9.828 & 7.654 & 1.050 & 0.232 & 1.019 \\
\hline II-1 & & LICK & $18: 36: 23.89$ & $-23: 52: 43.5$ & 13.585 & 12.043 & 10.367 & 8.204 & 1.003 & 0.321 & 1.089 \\
\hline II-31 & & LICK & 18:36:09.93 & $-23: 52: 52.6$ & 13.436 & 11.927 & 10.234 & 8.124 & 1.015 & 0.210 & 1.026 \\
\hline II-96 & & MCD & 18:36:17.24 & $-23: 54: 11.3$ & 13.134 & 11.604 & 9.879 & 7.786 & 1.033 & 0.219 & 1.042 \\
\hline II-104 & 71 & UVES & $18: 36: 07.72$ & $-23: 50: 55.4$ & 13.764 & 12.336 & 10.696 & 8.658 & 0.959 & 0.124 & 1.083 \\
\hline III-3 & 200006 & APO, MCD, UVES & $18: 36: 17.51$ & $-23: 54: 26.2$ & 12.912 & 11.107 & 9.188 & 6.783 & 1.175 & 0.563 & 1.039 \\
\hline III-12 & & AAT, MCD & 18:36:14.26 & $-23: 54: 31.1$ & 13.236 & 11.540 & 9.723 & 7.401 & 1.106 & 0.452 & 1.024 \\
\hline III-14 & & AAT, LICK & $18: 36: 15.10$ & $-23: 54: 54.6$ & 12.964 & 11.134 & 9.145 & 6.743 & 1.201 & 0.445 & 1.034 \\
\hline III-15 & & LICK & $18: 36: 15.61$ & $-23: 55: 01.2$ & 13.057 & 11.362 & 9.484 & 7.138 & 1.106 & 0.403 & 1.007 \\
\hline III-25 & 200104 & UVES & $18: 36: 20.16$ & $-23: 55: 53.9$ & 13.873 & 12.688 & 11.207 & 9.397 & 0.850 & 0.026 & 1.014 \\
\hline III-33 & 61 & UVES & $18: 36: 20.13$ & $-23: 56: 45.4$ & 13.640 & 12.249 & 10.632 & 8.618 & 0.964 & 0.117 & 1.018 \\
\hline III-35 & 200076 & UVES & $18: 36: 20.51$ & $-23: 56: 24.4$ & 13.738 & 12.404 & 10.828 & 8.854 & 0.930 & 0.098 & 1.042 \\
\hline III-47 & & APO & $18: 36: 14.80$ & $-23: 55: 15.7$ & 13.699 & 12.385 & 10.848 & 8.979 & 0.904 & 0.128 & 0.992 \\
\hline III-50 & 224 & UVES & $18: 36: 13.54$ & $-23: 54: 54.5$ & 14.699 & 13.493 & 12.066 & 10.319 & 0.819 & 0.115 & 0.981 \\
\hline III-52 & 200025 & LICK, UVES & $18: 36: 10.18$ & $-23: 54: 21.8$ & 13.238 & 11.526 & 9.732 & 7.459 & 1.081 & 0.549 & 1.016 \\
\hline III-96 & & AAT & 18:36:02.20 & $-23: 56: 50.1$ & 13.511 & 12.138 & 10.575 & 8.583 & $\ldots$ & $\ldots$ & 0.996 \\
\hline IV-20 & 51 & AAT, UVES & $18: 36: 25.99$ & $-23: 55: 58.3$ & 13.622 & 12.071 & 10.383 & 8.164 & 1.021 & 0.336 & 1.036 \\
\hline IV-59 & 200043 & UVES & $18: 36: 32.16$ & $-23: 56: 03.9$ & 13.408 & 11.927 & 10.233 & 8.152 & 1.002 & 0.144 & 1.139 \\
\hline IV-68 & 221 & UVES & $18: 36: 32.97$ & $-23: 54: 59.0$ & 14.682 & 13.490 & & 10.222 & 0.833 & 0.041 & 1.065 \\
\hline IV-76 & & AAT & $18: 36: 35.41$ & $-23: 54: 39.9$ & 13.709 & 12.299 & 10.790 & 8.852 & 0.916 & 0.289 & 1.066 \\
\hline IV-88 & & AAT & $18: 36: 44.97$ & $-23: 54: 57.3$ & 13.718 & 12.228 & 10.608 & 8.500 & $\ldots$ & $\ldots$ & 1.065 \\
\hline IV-97 & 200083 & UVES & $18: 36: 41.06$ & $-23: 58: 18.9$ & 12.799 & 11.043 & 9.065 & 6.759 & $\ldots$ & $\ldots$ & 1.102 \\
\hline IV-102 & & AAT, LICK, MCD & $18: 36: 36.19$ & $-23: 59: 38.9$ & 12.881 & 11.051 & 9.111 & 6.769 & $\ldots$ & $\ldots$ & 0.986 \\
\hline $\mathrm{V}-2$ & & LICK & $18: 36: 28.02$ & $-23: 55: 01.6$ & 13.260 & 11.498 & 9.674 & 7.276 & 1.160 & 0.494 & 1.036 \\
\hline $\mathrm{C}$ & & MCD & 18:36:10.21 & $-23: 48: 44.0$ & 13.309 & 11.309 & 9.253 & 6.737 & $\ldots$ & $\ldots$ & 0.989 \\
\hline C513 & & APO & $18: 35: 50.02$ & $-23: 57: 40.6$ & 13.052 & 11.356 & 9.567 & 7.359 & $\ldots$ & $\ldots$ & 1.072 \\
\hline
\end{tabular}

Notes. ${ }^{(a)}$ When available, we used the identification scheme of Arp \& Melbourne (1959) or its extension by Lloyd-Evans (1975), with Roman numerals I-IV for the quadrants and V for the center. If no other name is available, we used identifications from Cudworth (1986), preceded by C. These names are entered into the SIMBAD database (http://simbad.u-strasbg.fr/simbad/) as "NGC 6656 abbb", where "a" is the roman numeral and "bbb" is the number; for example, star IV-97 can be found as NGC 66564097 in SIMBAD. ${ }^{(b)}$ See text for definitions. ${ }^{(c)}$ Stetson photmetric database. ${ }^{(d)}$ 2MASS database. ${ }^{(e)}$ Richter et al. (1999).

correction to the observed $(B-V)$ values of red giant(s) that occur near the same spatial location in the cluster. Finally, we adopted the Cardelli et al. (1989) recommended ratio of total to selective extinction, $R_{V}=3.1$, to convert $E(B-V)$ to $A_{V}$ values. We also used the Cardelli et al. extinction curve to perform extinction corrections to the other colours.

\section{Model atmosphere parameters}

Our spectroscopic analysis of M 22 giant stars was conducted with the local thermodynamic equilibrium (LTE) line analysis code MOOG (Sneden 1973). In order to keep the present analysis consistent with that of M09, we used interpolated model atmospheres from the grid of Kurucz (1992). Those models include convective overshooting, which is not part of subsequent grids in this series (e.g., Castelli \& Kurucz 2004). In Sect. 4.2 we will comment on the (small) effect of using these overshoot models in our analysis.

Atmospheric parameters for these models were estimated from Fe spectral lines. Effective temperatures $T_{\text {eff }}$ were derived by removing trends in $\mathrm{Fe}$ I abundances with excitation potential, and the microturbulent velocities $\xi_{\mathrm{t}}$ were set by removing trends with EW. Gravities were determined by satisfying the ionization equilibrium between $\mathrm{Fe}$ I and $\mathrm{Fe}$ II abundances. This process was done iteratively until a final interpolated model was obtained. In this manner we specified model atmosphere parameters entirely based on the spectra; thus they are independent of photometric information. This is an important advantage when analyzing M 22, with its star-to-star reddening variations. More details about the line list, atmospheric parameters determination, and abundance measurements can be found in Marino et al. (2008) and M09.

The adopted atmospheric parameters for the program stars, together with the telescope source of the spectra, are listed in Table 3. By comparing the atmospheric parameters for the same stars observed with different sources, no evidence for systematic offsets have been found.

To investigate internal uncertainties related to the atmospheric parameters we applied the same procedure used in Marino et al. (2008) and M09 to which we refer the reader for a more detailed description. The procedure was applied separately for spectra taken with different instruments because of the different quality of the data. Briefly, first we calculated, for the stars, the errors associated with the slopes of the best least squares fit 
A. F. Marino et al.: The two metallicity groups in M22
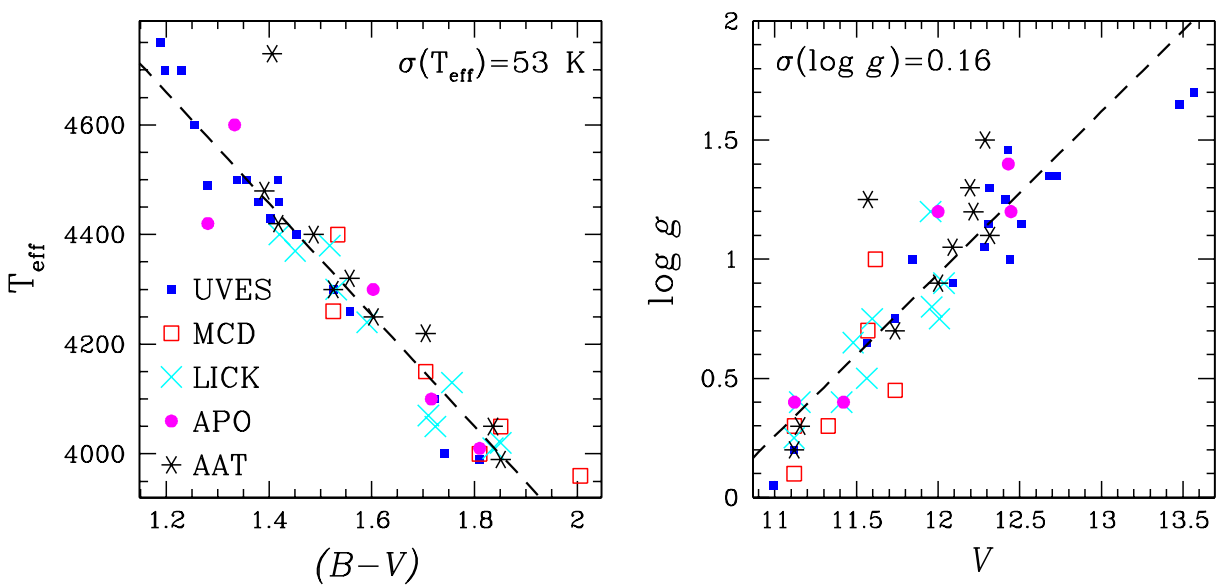

Fig. 1. Adopted $T_{\text {eff }}$ and $\log g$ as a function of $(B-V)$ colour and $V$ mag respectively. Different data-sets were represented by different symbols (as quoted in the left panel).

in the relations between Fe I abundance vs. E.P. The average of the errors corresponds to the typical error on the slope. Then, we selected, for each set of data observed at different telescopes, a star at intermediate temperature. For these stars, we fixed the other parameters and varied the temperature until the slope of the line that best fits the relation between abundances and E.P. became equal to the respective mean error. These differences in temperature represent an estimate of the error in temperature itself. A similar procedure was applied for $\xi_{\mathrm{t}}$, but using the relation between abundance and EWs. For gravities, determined by imposing the ionization equilibrium for iron, we considered the averaged uncertainties $\overline{\sigma_{\text {star }}[\mathrm{FeII} / \mathrm{H}]}$ and $\overline{\sigma_{\text {star }}[\mathrm{FeI} / \mathrm{H}]}$ (where $\sigma_{\text {star }}[\mathrm{Fe} / \mathrm{H}]$ is the dispersion of $\mathrm{Fe}$ I and $\mathrm{Fe}_{\text {II }}$ abundances derived by the various spectral lines in each spectrum as given by MOOG, divided by $\sqrt{N_{\text {lines }}-1}$ ), and varied the gravity of the representative stars such that the relation:

$[\mathrm{FeI} / \mathrm{H}]-\overline{\sigma_{\text {starr }}[\mathrm{FeI} / \mathrm{H}]}=[\mathrm{FeII} / \mathrm{H}]+\overline{\sigma_{\text {star }}[\mathrm{FeII} / \mathrm{H}]}$

was satisfied.

The resulting atmospheric parameter uncertainties for different data sets are listed in Table 4 together with the abundance uncertainties (see Sect. 4.2).

In order to understand the uncertainties associated with our spectroscopically-derived temperatures and gravities, we first tested the assumption that RGB stars with the same photometric properties ought to have the same $T_{\text {eff }}$ and $\log g$ values. In Fig. 1 we plot our adopted $T_{\text {eff }}$ as a function of the $(B-V)$ colour, and $\log g$ as a function of $V$. The mean trends were computed with linear least square regressions. The rms of the differences between individual points and the mean curve is quoted in each panels of Fig. 1, and are not dissimilar from our estimated $T_{\text {eff }}$ and $\log g$ uncertainties. These values are an estimate of the differences that we expect in temperatures and gravities among stars with similar magnitude and colours.

In Fig. 2 we compare our spectroscopically-derived model atmosphere parameters with these quantities derived by other methods. In the upper-left panel we show the comparison of our effective temperatures, determined from $\mathrm{Fe}_{\mathrm{I}}$ excitation equilibrium $\left(T_{\text {eff }}\right)$, with the ones obtained from photometry. Photometric temperatures $T_{(V-K)}$ have been derived from the colours $(V-K)$ corrected for differential reddening (as explained in Sect. 2), by using the calibrations of Alonso et al. (1999, 2001), assuming a mean reddening of $E(B-V)=0.34$ (Harris 1996). The scatter of the points around the line of perfect agreement is $\sim 50 \mathrm{~K}$, that is what we expect from observational errors.
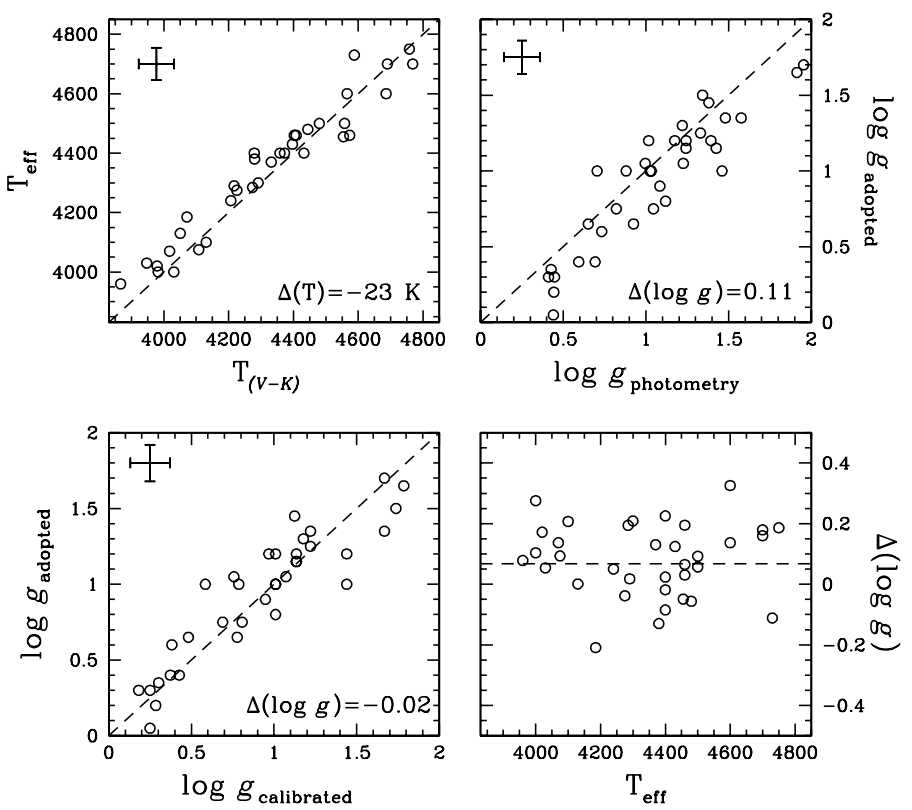

Fig. 2. Upper-left panel: adopted temperatures derived from the excitation potential equilibrium $\left(T_{\text {eff spec }}\right)$ as a function of photometric temperatures obtained from colours $(V-K)$. Upper-right panel: adopted gravities from ionization equilibrium ( $\left.\log g_{\text {adopted }}\right)$ as a function of gravities obtained from the estimated mass ( $\left.\log g_{\text {photometry }}\right)$, radius, and adopting a distance modulus of $(m-M)_{V}=13.60$ (Harris 1996). Lower-left panel: adopted gravities versus the calibrated $T_{\text {eff }}-\log$ g relation from Kučinskas et al. (2006). The dashed line in these three panels indicates perfect agreement. The mean difference between the adopted values and the comparison ones has been quoted in each panel (as $\Delta$ (comparisonadopted)). Lower-right panel: distances $\Delta(\log g)$ of each point in the upper panel, in the $\log g_{\text {photometry }}-\log g_{\text {adopted }}$ plane, from the line of perfect agreement, as a function of the adopted $T_{\text {eff }}$.

Since we used colours corrected for differential reddening, we have minimized this effect in the derivation of $T_{(V-K)}$.

The upper-right panel shows our spectroscopic gravities determined from $\mathrm{Fe}$ I/Fe II ionization equilibrium ( $\log g_{\text {adopted }}$ ) as a function of gravities $\log g_{\text {photometry }}$ that were obtained with standard relations by using $T_{(V-K)}$, bolometric corrections from Alonso et al. (1999), and a distance modulus of $(m-M)_{V}=$ 13.60 (Harris 1996). The dispersion around the line of perfect agreement is $\sim 0.20$ dex, and is in both axis, i.e. partly in $(V-K)$ due to uncertain reddening (which is much more in $(V-K)$ than 
$(B-V))$ and the uncertainty introduced by the NLTE effects in

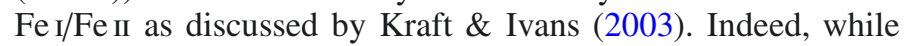
there are good reasons to base $[\mathrm{Fe} / \mathrm{H}]$ on the lines of Fe II $(\mathrm{Kraft}$ \& Ivans 2003) our resolution limits the number of unblended Fe II lines so that it is necessary to base $[\mathrm{Fe} / \mathrm{H}]$ on lines of $\mathrm{Fe}$ I. However, the scatter of points in the gravity plane in the upper panel of Fig. 2 from the line of perfect agreement exhibits no obvious $T_{\text {eff }}$ dependence. This is emphasized in the gravity difference plot in lower-right panel of the figure. Finally, in the lower-left panel we show our adopted spectroscopic log $g$ values compared with estimates based on spectroscopic gravity calibrations of GCs provided by Kučinskas et al. (2006).

These tests demonstrate that our estimates of the atmospheric parameters are quite reliable and that NLTE effects may not be important in determining these quantities. Of course, some offsets between the photometric and spectroscopic parameters may be present, as it seems for temperature with spectroscopic values $\sim 20-30 \mathrm{~K}$ higher than $T_{V-K}$, but these offsets are comparable with our uncertainties.

\section{Abundance derivations}

Using the model atmospheres and analysis code described in Sect. 3, we determined abundances for Fe, $\alpha$ elements $(\mathrm{Mg}, \mathrm{Si}$, $\mathrm{Ca}, \mathrm{Ti}), p$-capture elements $(\mathrm{C}, \mathrm{N}, \mathrm{O}, \mathrm{Na}$, and $\mathrm{Al}), \mathrm{Fe}$-peak elements $\mathrm{Cu}$ and $\mathrm{Zn}$, and several $n$-capture elements (Y, Zr, Ba, La, $\mathrm{Nd}, \mathrm{Eu}$ ). To accomplish this we employed EWs for Fe, the $\alpha$ elements, Y, Ba, and Nd. For C, N, O, Na, Zr, La, Eu, Zn and Cu, because of significant atomic blends, isotopic/hyperfine structure issues, or weak molecular band $(\mathrm{CN})$ contamination, we derived abundances by comparing synthetic and observed spectra. We list the results for light and heavy elements in Tables 5 and 6 respectively. In this section we comment on the transitions that we used, and on uncertainties in the resulting abundances.

\subsection{Spectral features}

Proton-capture elements: We determined $\mathrm{Na}$ abundances from spectral synthesis of the Na I doublets at $5680 \AA$ and $6150 \AA$, and $\mathrm{O}$ abundances from the synthesis of the forbidden [O I] line at $6300 \AA$. Aluminum was determined from EWs of the doublet at $6667 \AA$. For the UVES data, the $\mathrm{O}, \mathrm{Na}$, and $\mathrm{Al}$ abundances are those reported in M09. We applied NLTE corrections from Lind et al. (2011b) to the $\mathrm{Na}$ abundances. These corrections are not available for gravities $\lesssim 1.00$. However our $\mathrm{Na}$ I line strengths are relatively insensitive to gravity. We determined NLTE corrections for the lowest gravity stars using the Lind et al. corrections for $\log g=1.0$, but future NLTE corrections for Na abundances will be welcome. In the following discussion both NLTE and LTE Na abundances will be presented.

For 14 (out of 35) stars we were able to determine $\mathrm{C}$ and $\mathrm{N}$ abundances. Carbon was measured from spectral synthesis of the CH G-band $\left(A^{2} \Delta-X^{2} \Pi\right)$ heads near 4314 and $4323 \AA$. Nitrogen was derived from synthesis of the $2-0$ band of the $\mathrm{CN}$ red system $\left(A^{2} \Pi-X^{2} \Sigma\right)$ near $8005 \AA$ (available for the MCD spectra), and from the CN blue system $\left(B^{2} \Sigma-X^{2} \Sigma\right)$ bandhead at $\sim 4215 \AA$ (for UVES data). The synthesis linelist for the blue $\mathrm{CN}$ band is described in Hill et al. (2002). The linelists for the $\mathrm{CH}$ band and the $\mathrm{CN}$ red system were provided by $\mathrm{B}$. Plez $(\mathrm{CH}$ band, priv. comm.), and V. Smith (CN band, priv. comm.). As an example of the molecular band calculations, in Fig. 3 we show synthetic/observed spectral matches of the $\mathrm{CN}$ red system for two stars IV-102 and III-3 that have nearly identical atmospheric

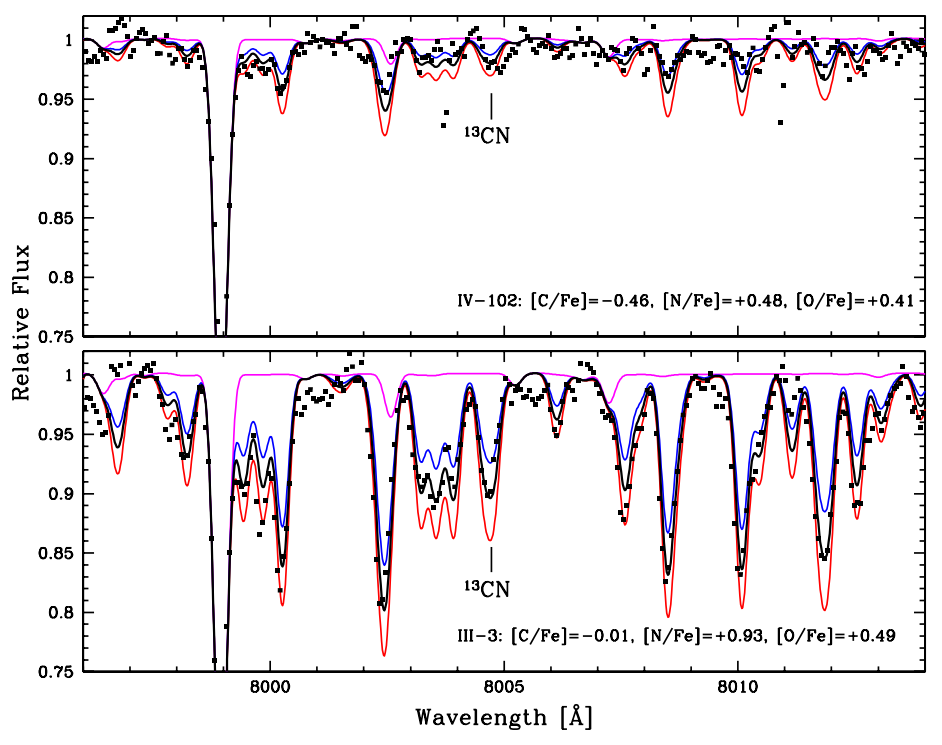

Fig. 3. Spectral region around the $\mathrm{CN}$ features at $8000 \AA$ from $\mathrm{McD}$ data for the two stars IV-102 (upper panel) and III-3 (lower panel). The observed spectrum is shown as points. Synthetic spectra with $\mathrm{N}$ value of best fit (black line), with no $\mathrm{N}$ (magenta line), and with $\Delta(\mathrm{N})= \pm 0.2 \mathrm{dex}$ deviations from the best fit (blue and red lines) are superimposed to the spectrum of the stars.

parameters $T_{\mathrm{eff}}, \log g$, and $\xi_{\mathrm{t}}$. Superimposed on the observed spectra are synthetic models at constant $\mathrm{C}, \mathrm{O}$, while varying $\mathrm{N}$ around the best fit value of $\Delta \mathrm{N}= \pm 0.20$ dex. The star IV-102, similarly to some other stars in our sample, has very weak features of CN bands; hence we could estimate only an upper limit to its $\mathrm{N}$ abundance.

Of course, in the $\mathrm{C}$ abundance computations we used the previously-determined $\mathrm{O}$ contents of each star, and for $\mathrm{N}$, both observed $\mathrm{C}$ and $\mathrm{O}$ abundances needed to be employed. Unfortunately we could measure $\mathrm{C}$ and $\mathrm{N}$ only for MCD and UVES data. Spectra obtained with several of the other instruments do not have sufficient $S / N$ in the spectral region around the $\mathrm{CH}$ bands to determine meaningful values or limits for $\mathrm{C}$. For UVES only a sub-sample of six stars have available data in the spectral range covering molecular bands of $\mathrm{CN}$ and $\mathrm{CH}$.

Isotopic ratios ${ }^{12} \mathrm{C} /{ }^{13} \mathrm{C}$ were derived for the six MCD stars, since relatively strong and isolated features of ${ }^{12} \mathrm{CN}$ and ${ }^{13} \mathrm{CN}$ are available for the $2-0$ band of the $\mathrm{CN}$ red system. First, several iterations of synthesis were done to obtain a satisfactory match to the strengths of the ${ }^{12} \mathrm{CN}$ features, after which syntheses were calculated with different values of the ${ }^{12} \mathrm{C} /{ }^{13} \mathrm{C}$ ratio. The isotopic ratio was derived from several ${ }^{13} \mathrm{CN}$ features, with the highest weight given to the blended triplet of lines at $8004.7 \AA$. Other features in the same "window" provide a check on the ratio.

$\alpha$ elements: We determined abundances from EWs of the same $\mathrm{Si}, \mathrm{Mg}$, Ca, and Ti (I and II) lines used in M09. We measured $\mathrm{Ca}$ and $\mathrm{Ti}$ from usually about 10 transitions, while for $\mathrm{Mg}$ and $\mathrm{Si}$ we had few lines, typically about four for $\mathrm{Si}$, and one or two for $\mathrm{Mg}$.

Heavy Fe-peak elements: We determined abundances for $\mathrm{Cu}$ from synthesis of the $\mathrm{Cu}$ I lines at 5105, 5218, and $5782 \AA$. Both hyperfine and isotopic splitting were included in the analysis, with well-studied spectral line component structure from the Kurucz $(2009)^{5}$ compendium. Solar-system isotopic fractions were assumed in the computations: $f\left({ }^{63} \mathrm{Cu}\right)=0.69$ and

5 Available at: http://kurucz.harvard.edu/ 


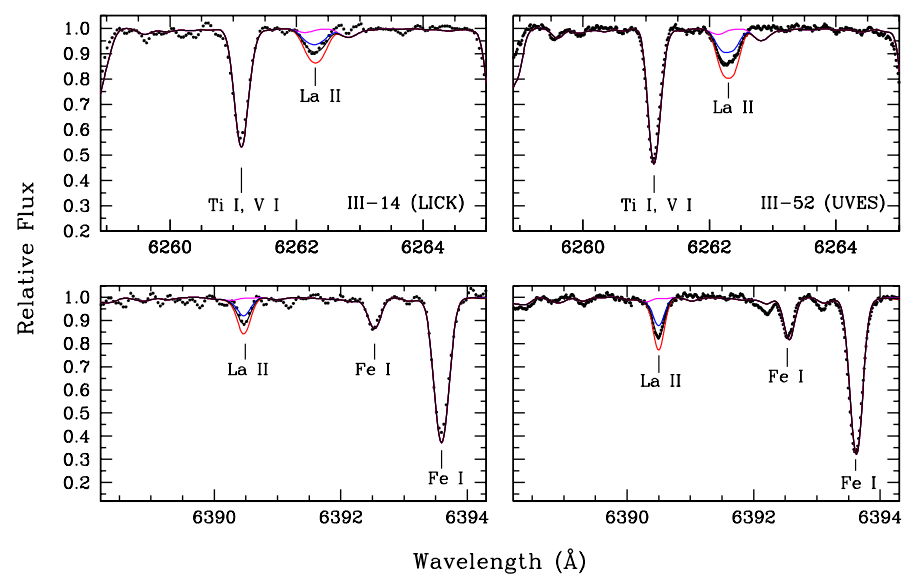

Fig. 4. Observed and synthetic spectra around the La lines at $6262 \AA$ and $6390 \AA$ for the $s$-poor star III-14 observed at LICK (left panels), and the $s$-rich star III-52 observed with UVES (right panels). In each panel the points represent the observed spectrum. The magenta line is the spectrum computed with no contribution from La II; the black line is the best-fitting synthesis (with the La abundance given in Table 6); and the red and blue lines are the syntheses computed with La abundances altered by \pm 0.2 dex from the best value.

$f\left({ }^{65} \mathrm{Cu}\right)=0.31$. For $\mathrm{Zn}$ we analyzed the $\mathrm{Zn}$ I lines at 4722 and $4810 \AA$ A. These lines have no significant hyperfine or isotopic substructures, and were treated as single absorbers in our syntheses. However, the $S / N$ of our spectra that extend down to $4722 \AA$ is poor, thus yielding larger abundance uncertainties.

Neutron-capture elements: For UVES data, $\mathrm{Y}$ and $\mathrm{Ba}$ are from M09, to which we add new measurements for $\mathrm{Zr}, \mathrm{La}, \mathrm{Nd}$, and Eu. Zirconium abundances for UVES data were calculated by M09 from EWs. Here we determine Zr from spectral synthesis of just the $5112 \AA \mathrm{Zr}$ II line. Hence, to homogeneously analyze our data, the UVES $\mathrm{Zr}$ abundances were re-determined with syntheses.

We determined Y, Ba, and Nd contents from EWs of isolated spectral lines. For Ba abundances we employed the 5853, 6141, and $6496 \AA$ Ba II lines. Since these lines have (very narrow) hyperfine and isotopic substructures, and suffer blending by other atomic species to greater or lesser degrees, we used a blendedline EW analysis option in our synthesis code.

Lanthanum abundances were derived from spectral synthesis of the La II lines at 6262, 6390, and $6774 \AA$ A. Hyperfine splitting for the 6262 and $6390 \AA$ lines was taken into account with the laboratory data from Lawler et al. (2001a). Hyperfine data are not available for the $6774 \AA$ line, but it is weak enough that no substantial abundance error results from treating the line as a single absorber. As examples of La syntheses, in Fig. 4 we show the 6262 and $6390 \AA$ lines in the LICK spectrum of star III14 (left panels) and in the UVES spectrum of star III-52 (right panels). These stars were chosen for display because they have nearly the same $T_{\text {eff }}$ and $\log g$ values, but have contrasting La line strengths.

For Eu we computed spectral syntheses of the Eu II line at $6645 \AA$, considering the hyperfine and isotopic splitting structure given in Lawler et al. (2001b). We did not obtain Eu from AAT data since those spectra do not cover the $6645 \AA$ spectral region. Due to the poor $S / N(<30)$ and line crowding we could not obtain reliable abundances from stronger Eu II lines in the blue-violet region.

\subsection{Abundance uncertainties}

Stars with repeated observations, for which we reported model atmospheres and abundances derived from different sources, suggest good agreement (within observational errors) of their results. For these stars, we will employ the averaged abundance results in subsequent discussions.

To verify how model atmosphere uncertainties influence the derived chemical compositions, we repeated the abundance derivations for one representative star at intermediate temperature for each set of data. For this exercise we changed only one atmospheric parameter each time. Results of these calculations are listed in Table 4. Assuming that the atmospheric parameter uncertainties are uncorrelated, we estimate total sensitivities for absolute abundances to be typically $\sim 0.15-0.20$. The abundance ratios $[\mathrm{X} / \mathrm{Fe}]$ have sensitivities of $\sim 0.05-0.10$ for LICK and MCD spectra, and slightly higher for APO and AAT data due to their lower resolution (see Table 4).

An additional source of abundance internal errors is the uncertainty in the EW measurements. In the case of Fe this contribution is small since a large number of transitions (typically $N_{\mathrm{Fe} 1} \simeq 40$ ) are available. The uncertainty can be estimated as $\sigma_{E W} / \sqrt{N_{\mathrm{Fe} \text { I }}-1}$, which on average we estimated as $\sim 0.02 \mathrm{dex}$ for all data sources. However, for those species with only few (or even one) transitions, such as $\mathrm{Mg}$ I and $\mathrm{Si} \mathrm{I}$, the error introduced by EWs measurements became important, $\sim 0.10$ dex. Particular caution should also be noted for $\mathrm{Ba}$ abundances that have been measured from only strong and blended lines.

In Table 4 we do not list the sensitivities of either $\mathrm{C}$ or $\mathrm{N}$ on the atmospheric parameters. The dominant source of uncertainty for the abundances derived from molecular bands is the continuum placement. For $\mathrm{C}$ the values we obtained for the two $\mathrm{CH}$ bandheads generally agree with each other within $\sim 0.15 \mathrm{dex}$, and we adopted the average of the two measurements as our final $\mathrm{C}$ abundance. In the case of $\mathrm{N}$, the continuum placement errors are not critical in the the $\mathrm{CN}$ red system available for MCD data. It could became important for the $\mathrm{CN}$ band at $\sim 4215 \AA$ that we used for the UVES spectra, however the relatively high $S / N$ ( 50-60) around the $\mathrm{CN}$ band of these data served to reduce such problems. Random uncertainties for $[\mathrm{C} / \mathrm{Fe}]$ and $[\mathrm{N} / \mathrm{Fe}]$ are estimated to be 0.10 and 0.20 dex, respectively.

Recall from Sect. 3 that we have used model atmospheres from the Kurucz (1992) grid, which include convective overshooting. To test the sensitivity of our abundances to this effect, we repeated the analysis using models without overshooting (Castelli \& Kurucz 2004) for few stars representative of the entire range in temperature. The main systematic results of this exercise are: (a) a decrease of $\lesssim 0.10$ in $\log g$ values; (b) a decrease in $[\mathrm{Fe} / \mathrm{H}]$ of $\sim 0.05$; (c) an increase in $[\mathrm{N} / \mathrm{Fe}]$ of $\sim 0.10 \mathrm{dex}$; and (d) negligible effect on other $[\mathrm{X} / \mathrm{Fe}]$ abundance ratios. Overshooting in the model atmospheres does not materially affect our results.

As a final estimate of errors associated with each abundance measurement, we assume the rms of the abundances of stars with the same chemical properties. This error includes both the errors introduced by atmospheric uncertainties, and errors due to EW measurements. Further details on how the stars with the same chemical properties were selected are given in Sect. 5 .

\section{Abundance results}

In this section, we consider our abundance results of $n$-capture, light, and $\alpha$ elements in M22, expanding on the discussion of M09. 

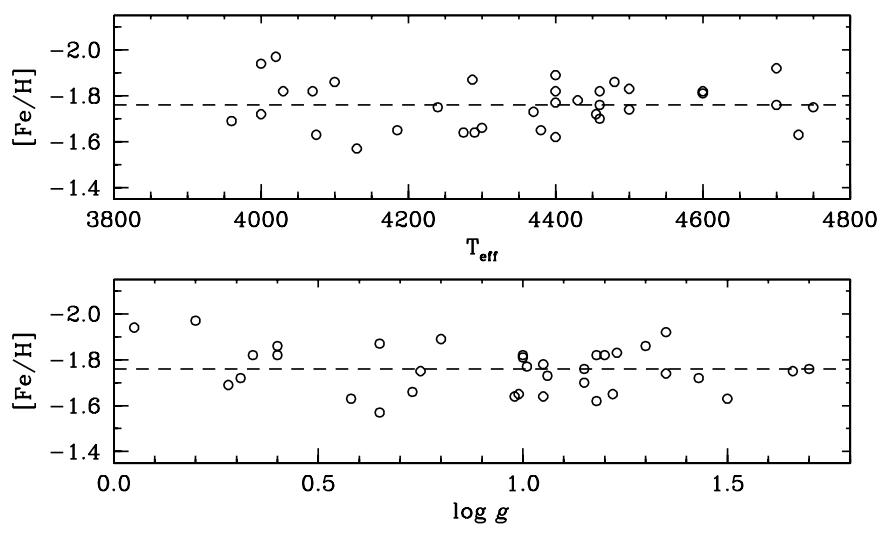

Fig. 5. Correlations of derived $[\mathrm{Fe} / \mathrm{H}]$ metallicities as functions of effective temperature (top panel) and gravity (bottom panel). The dashed line in each panel indicates the mean from the complete 35-star sample, $\langle[\mathrm{Fe} / \mathrm{H}]\rangle=-1.76$.

For our entire 35-star sample, we obtain a mean metallicity of $[\mathrm{Fe} / \mathrm{H}]=-1.76 \pm 0.02 \operatorname{dex}(\sigma=0.10)$. However, this simple mean obscures the fact that the total metallicity spread is more than a factor of two: $-1.57 \geq[\mathrm{Fe} / \mathrm{H}] \geq-1.97$, a range that cannot be explained by observational/analytical uncertainties. This point was demonstrated previously in M09 and in DC09. There are four stars in common between this work and DC09, who used intermediate resolution spectra at the $\mathrm{Ca}$ II triplet to derive $[\mathrm{Fe} / \mathrm{H}]$ values for $41 \mathrm{M} 22$ red giants. For these stars the mean difference in $[\mathrm{Fe} / \mathrm{H}]$, in the sense of this paper minus DC09, is small: $-0.01 \pm 0.04$ dex (sigma 0.09 dex). In Fig. 5 we plot individual $[\mathrm{Fe} / \mathrm{H}]$ values as functions of $T_{\text {eff }}$ and $\log g$. It is clear that there are no metallicity trends with either parameter; the scatter is the same at all M 22 giant branch positions. Below we consider the metallicity spread in concert with other M22 abundance anomalies.

A summary of our results for the 18 non-Fe species is displayed in Fig. 6, where we show relative abundances $[\mathrm{X} / \mathrm{Fe}]$ as a function of $[\mathrm{Fe} / \mathrm{H}]$. The numerical ranges of both quantities are the same in all panels of this figure so that one can compare the variations of different elements with changing metallicity in M22. The figure organization differentiates between the "lighter" elements $(Z<26$, shown in the two left-hand columns of panels), and the "heavier" elements (shown in the two righthand columns). We have represented stars belonging to two different metallicity groups in M 22 with different symbols: blue crosses for more metal-poor stars, and red filled circles for less metal-poor stars. We will justify and expand this distinction in Sect. 5.1. The error bar in each panel (and in the next figures of this paper) is an estimate of the uncertainty associated with individual abundance measures, calculated as the rms of the abundances of stars in the same metallicity group (see Sect. 4.2). For this estimate we used values from the more metal-poor group only. Of course, this is an overestimate of the error if intrinsic abundance variations are present in each group. The light protoncapture elements $(\mathrm{C}, \mathrm{N}, \mathrm{O}, \mathrm{Na}$, and $\mathrm{Al})$ exhibit intrinsic abundance variations within each metallicity group (see Sect. 5.4). For these elements, we calculated the rms for stars in metal-poor group that have $[\mathrm{Na} / \mathrm{Fe}]<+0.2$ dex.

Among the elements investigated in this paper, we found a small abundance trend with temperature for $\mathrm{Cu}, \mathrm{Zn}$, and Y relative to Fe. However, they affect in a similar way both M22 metallicity groups, and may possibly explain the some of the internal scatter of these abundances in the two groups, but they do not influence our basic results. In the next few subsections we consider the abundance trends among and between elements of different nucleosynthetic groups.

\subsection{The neutron-capture elements}

As described in the previous section, we follow M09 in using different symbols in Fig. 6 and subsequent figures to segregate stars into two metallicity groups. But Fig. 6 clearly suggests that relative $n$-capture abundances also vary with metallicity. Abundances of just $\mathrm{Y}, \mathrm{Zr}$, and $\mathrm{Ba}$ were reported in our earlier work. The solar-system abundances of these three elements are due overwhelmingly to the $s$-process: Y $72 \% ; \mathrm{Zr}$ $81 \%$, and $\mathrm{Ba} 85 \%$ (e.g., Table 10 of Simmerer et al. 2004). Therefore M09 called stars " $s$-rich" if $[\mathrm{Y} / \mathrm{Fe}]>0$ and " $s$-poor" if $[\mathrm{Y} / \mathrm{Fe}]<0$. This suggested link between $n$-capture-rich stars in M 22 with the $s$-process is sensible but not definitive, because Y, $\mathrm{Zr}$, Ba also can be synthesized in the $r$-process (e.g, see the review by Sneden et al. 2008, which has references to individual $r$-process-rich stars).

Here we can make a cleaner test by comparing the abundances of elements with sharply contrasting solar-system $s$-process $/ r$-process origins: La (75\% $s$-process) and Eu (only $3 \% s$-process). In Fig. 7 we show abundance ratios $[\mathrm{Eu} / \mathrm{Fe}]$, $[\mathrm{La} / \mathrm{Fe}]$, and $[\mathrm{La} / \mathrm{Eu}]$ as functions of $[\mathrm{Fe} / \mathrm{H}]$. Panels (a) and (b) are enlargements of their respective panels shown in Fig. 6. From these data one sees that relative abundances of Eu have no dependence on metallicity, while those of La exhibit a positive correlation. Therefore, in agreement with Da Costa \& Marino (2010), there is no doubt that the variations in $[\mathrm{La} / \mathrm{Fe}]$ are due to variations in amounts of $s$-process material.

We have employed spectrum syntheses to derive the La and $\mathrm{Eu}$ abundances, because the spectral features of both La II and Eu II have significant hyperfine substructure, and the Eu II lines also have isotopic splitting. However, the differences between the $s$-rich and $s$-poor groups as defined by M09 can be easily seen in the spectra without any detailed analyses. In Fig. 8 we show the La and Eu transitions in stars with similar atmospheric parameters but very different derived $[\mathrm{Fe} / \mathrm{H}]$ and $[\mathrm{La} / \mathrm{Eu}]$ ratios. The $s$-rich star III-3 $\left(T_{\text {eff }} / \log g / \xi_{\mathrm{t}} /[\mathrm{Fe} / \mathrm{H}]=\right.$ $4000 / 0.30 / 2.20 /-1.72$, Table 3) clearly has much stronger La lines than does the $s$-poor star IV-102 (4020/0.20/2.20/-1.97), while its Eu lines are perhaps even weaker than those of IV-102. Inspection of other contrasting pairs of stars yields the same conclusion. The $\mathrm{La} / \mathrm{Eu}$ ratios are very different in the $s$-poor and $s$-rich stars.

In panel (c) of Fig. 7 we plot the $[\mathrm{La} / \mathrm{Eu}]$ ratios of our sample; here the separation between $s$-rich and $s$-poor stars is even more clear than in the $[\mathrm{La} / \mathrm{Fe}]$ ratios shown in panel (b). For the entire M 22 sample, $\langle[\mathrm{La} / \mathrm{Eu}]\rangle=-0.30$ (Table 7), and the gap between the smallest $[\mathrm{La} / \mathrm{Eu}]$ value of the $s$-rich stars and the largest $[\mathrm{La} / \mathrm{Eu}]$ value of the $s$-poor stars is nearly 0.2 dex. Therefore for the remainder of this paper we redefine $s$-rich stars as those with $[\mathrm{La} / \mathrm{Eu}]>-0.3$; filled red circles will be used to identify them in the figures. Similarly, the $s$-poor stars hereafter are those with $[\mathrm{La} / \mathrm{Eu}]<-0.30$; they will be plotted with blue crosses in the figures. This empirically-set dividing line between the two groups is indicated in panel (c) of Fig. 7. All s-rich stars also have $[\mathrm{La} / \mathrm{Fe}] \geq+0.2$, as we indicate with the line in panel (b).

With this defined division by $[\mathrm{La} / \mathrm{Eu}]$ ratio, 14 stars (40\% of the total sample) are s-rich and 21 stars $(60 \%)$ are $s$-poor. In Table 7 we give the mean abundances for the whole sample and for the $s$-rich and $s$-poor subsets. For two 


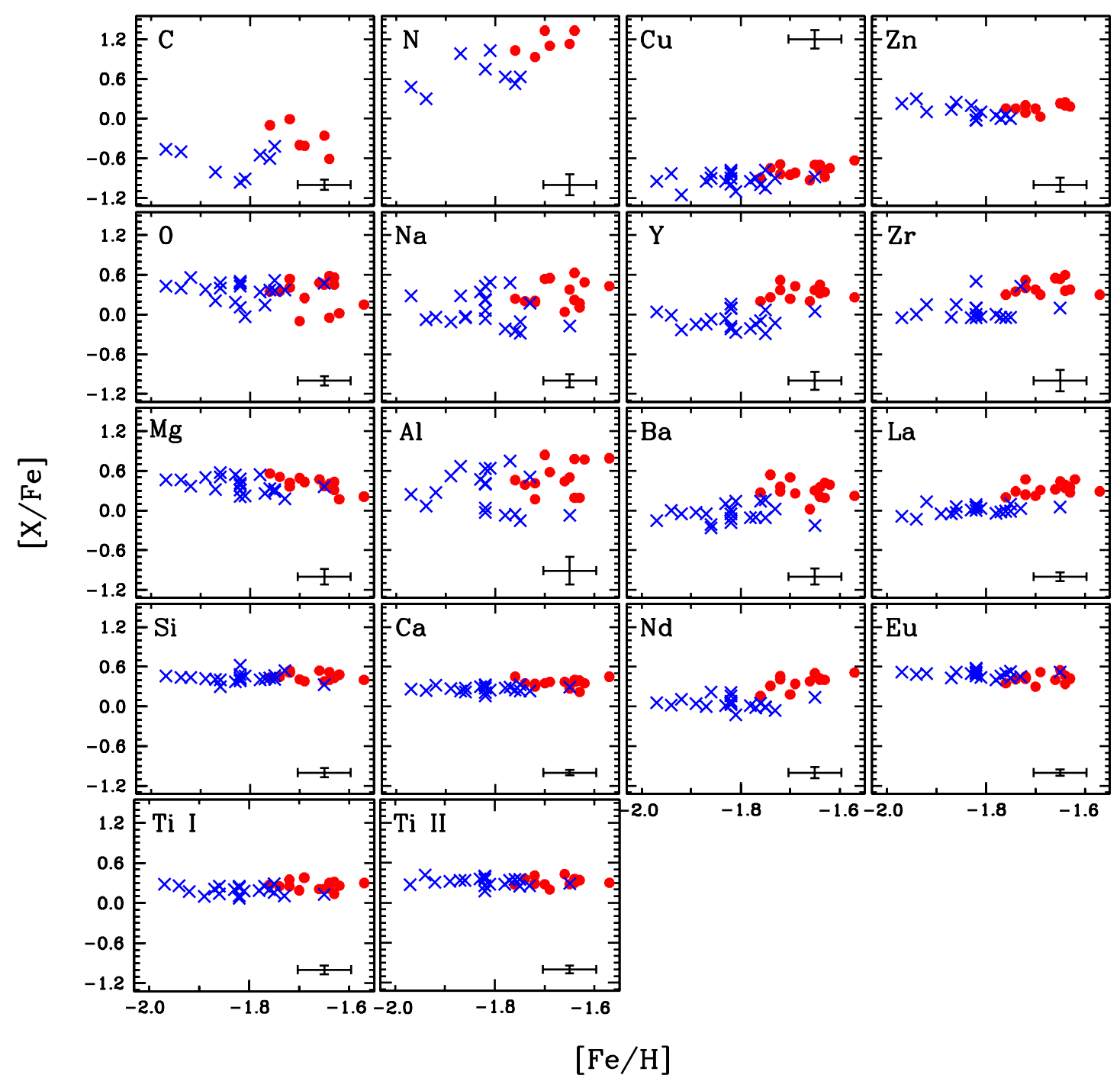

Fig. 6. Summary of the abundance results. For all 18 non-Fe species, their $[\mathrm{X} / \mathrm{Fe}]$ relative abundances are plotted versus their $[\mathrm{Fe} / \mathrm{H}] \mathrm{metallicities}$. The horizontal and vertical ranges are identical in all panels. Filled circles are used for stars with $s$-process enhancements and $\times$ symbols are for stars without such enhancements; see Sect. 5.1 for definitions of these two stellar groups. Error bars in each panel represent estimated errors for single measurements.

elements $\mathrm{A}$ and $\mathrm{B}$, we can define the difference in their abundance ratio between the $s$-rich and $s$-poor stars as $\Delta_{\text {poor }}^{\text {rich }}[\mathrm{A} / \mathrm{B}] \equiv$

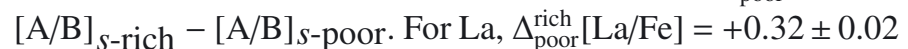
(Table 7). Nearly identical results are obtained for the other four $s$-process-dominated elements in our survey: $\Delta_{\text {poor }}^{\text {rich }}[\mathrm{Y} / \mathrm{Fe}]=$ $+0.41 \pm 0.04, \quad \Delta_{\text {poor }}^{\text {rich }}[\mathrm{Zr} / \mathrm{Fe}]=+0.34 \pm 0.05, \quad \Delta_{\text {poor }}^{\text {rich }}[\mathrm{Ba} / \mathrm{Fe}]=$ $+0.36 \pm 0.05$, and $\Delta_{\text {poor }}^{\text {rich }}[\mathrm{Nd} / \mathrm{Fe}]=+0.32 \pm 0.04$. This consistency is illustrated in Fig. 9, whose four panels show correlations of $[\mathrm{La} / \mathrm{Eu}]$ versus $[\mathrm{Y} / \mathrm{Eu}],[\mathrm{Zr} / \mathrm{Eu}],[\mathrm{Ba} / \mathrm{Eu}]$, and $[\mathrm{Nd} / \mathrm{Eu}]$. Note the relatively large star-to-star scatter in Ba abundances compared to other elements. This is due to difficulties associated with deriving reliable abundances for this species which, as described in Sect. 4.1, is represented by three lines that have hyperfine and isotopic splitting and are general saturated in M22 giant star spectra.

For each star we have formed average $s$-process-element abundance ratios $[s$-process $/ \mathrm{Fe}]$ and $[s$-process $/ \mathrm{Eu}]$, where $s$-process here represents the five elements $\mathrm{Y}, \mathrm{Zr}, \mathrm{Ba}, \mathrm{La}$, and $\mathrm{Nd}$ in most cases. In some cases we were not able to derive abundances for one or more of the $s$-process-elements; their means were formed from the available abundances. In
Fig. 10 we display these results. For the $s$-rich stars we derive $\langle[s$-process $/ \mathrm{Fe}]\rangle=+0.35 \pm 0.02(\sigma=0.06)$ and $\langle[s$-process $/ \mathrm{Eu}]\rangle=-0.07 \pm 0.02(\sigma=0.06)$, while for the $s$-poor stars we derive $\langle[s$-process $/ \mathrm{Fe}]\rangle=-0.01 \pm 0.01(\sigma=0.06)$ and $\langle[s$-process $/ \mathrm{Eu}]\rangle=-0.49 \pm 0.01(\sigma=0.05)$. These means and $\sigma$ values are also shown in Fig. 10. All the $n$-capture abundance data displayed in the bottom panel of Fig. 10 strongly support the notion of a bimodal separation between the $s$-rich and $s$-poor groups, as suggested by M09, with a typical [ $s$-process/Eu] difference of $\sim 0.4$ between the groups.

Taking the $[\mathrm{La} / \mathrm{Y}]$ abundance ratio as a [heavy-s/light- $s]$ indicator, we find that the $s$-rich stars show also slightly lower $[\mathrm{La} / \mathrm{Y}]$ abundance: $\Delta_{\text {poor }}^{\text {rich }}[\mathrm{La} / \mathrm{Y}]=-0.12 \pm 0.05$ (Table 7). This is suggestive of additional light- $s$ synthesis products from the progenitors to the $s$-rich stars.

The mean $[\mathrm{Fe} / \mathrm{H}]$ values for the $s$-poor and the $s$-rich groups are $\langle[\mathrm{Fe} / \mathrm{H}]\rangle_{s \text {-poor }}=-1.82 \pm 0.02$ and $\langle[\mathrm{Fe} / \mathrm{H}]\rangle_{s \text {-rich }}=-1.67 \pm 0.01$ respectively (see Table 7). The two groups have a different $[\mathrm{Fe} / \mathrm{H}]$ with a mean difference of $0.15 \pm 0.02$ dex, a $5 \sigma$ effect. The standard deviations of the $[\mathrm{Fe} / \mathrm{H}]$ values are 0.07 and 0.05 for the $s$-poor and $s$-rich which 
Table 7. Mean abundances.

\begin{tabular}{|c|c|c|c|c|c|c|c|c|c|c|c|c|}
\hline Abundance & \multicolumn{4}{|c|}{ total } & Mean & \multicolumn{3}{|c|}{$s$-poor } & Mean & \multicolumn{2}{|c|}{$s$-rich } & \# \\
\hline$[\mathrm{Fe} / \mathrm{H}]$ & -1.76 & 0.02 & 0.10 & 35 & -1.82 & 0.02 & 0.07 & 21 & -1.67 & 0.01 & 0.05 & 14 \\
\hline$[\mathrm{C} / \mathrm{Fe}]$ & -0.50 & 0.08 & 0.28 & 14 & -0.65 & 0.08 & 0.21 & 8 & -0.30 & 0.10 & 0.22 & 6 \\
\hline$[\mathrm{N} / \mathrm{Fe}]$ & 0.87 & 0.09 & 0.32 & 14 & 0.67 & 0.09 & 0.25 & 8 & 1.14 & 0.07 & 0.16 & 6 \\
\hline$[\mathrm{O} / \mathrm{Fe}]$ & 0.34 & 0.03 & 0.18 & 35 & 0.36 & 0.03 & 0.15 & 21 & 0.32 & 0.06 & 0.23 & 14 \\
\hline$[\mathrm{Na} / \mathrm{Fe}]_{\mathrm{NLTE}}$ & 0.17 & 0.04 & 0.25 & 35 & 0.08 & 0.05 & 0.24 & 21 & 0.31 & 0.05 & 0.19 & 14 \\
\hline$[\mathrm{Na} / \mathrm{Fe}]_{\text {LTE }}$ & 0.26 & 0.05 & 0.27 & 35 & 0.15 & 0.06 & 0.26 & 21 & 0.42 & 0.06 & 0.20 & 14 \\
\hline$[\mathrm{Mg} / \mathrm{Fe}]$ & 0.39 & 0.02 & 0.11 & 35 & 0.38 & 0.03 & 0.12 & 21 & 0.39 & 0.03 & 0.11 & 14 \\
\hline$[\mathrm{Al} / \mathrm{Fe}]$ & 0.38 & 0.05 & 0.29 & 31 & 0.29 & 0.07 & 0.30 & 18 & 0.50 & 0.07 & 0.24 & 13 \\
\hline$[\mathrm{Si} / \mathrm{Fe}]$ & 0.44 & 0.01 & 0.06 & 35 & 0.43 & 0.02 & 0.07 & 21 & 0.45 & 0.02 & 0.06 & 14 \\
\hline$[\mathrm{Ca} / \mathrm{Fe}]$ & 0.30 & 0.01 & 0.07 & 35 & 0.26 & 0.01 & 0.04 & 21 & 0.36 & 0.02 & 0.06 & 14 \\
\hline [TiI/Fe] & 0.22 & 0.01 & 0.07 & 35 & 0.19 & 0.02 & 0.06 & 21 & 0.26 & 0.02 & 0.07 & 14 \\
\hline [TiII/Fe] & 0.32 & 0.01 & 0.06 & 32 & 0.32 & 0.01 & 0.06 & 20 & 0.32 & 0.02 & 0.06 & 12 \\
\hline$[\mathrm{Cu} / \mathrm{Fe}]$ & -0.88 & 0.02 & 0.14 & 35 & -0.94 & 0.03 & 0.14 & 21 & -0.79 & 0.02 & 0.09 & 14 \\
\hline$[\mathrm{Zn} / \mathrm{Fe}]$ & 0.13 & 0.02 & 0.09 & 24 & 0.10 & 0.03 & 0.11 & 14 & 0.16 & 0.02 & 0.07 & 10 \\
\hline$[\mathrm{Y} / \mathrm{Fe}]$ & 0.07 & 0.04 & 0.24 & 32 & -0.08 & 0.03 & 0.13 & 20 & 0.33 & 0.03 & 0.10 & 12 \\
\hline$[\mathrm{Zr} / \mathrm{Fe}]$ & 0.21 & 0.04 & 0.22 & 30 & 0.07 & 0.04 & 0.16 & 18 & 0.41 & 0.03 & 0.11 & 12 \\
\hline$[\mathrm{Ba} / \mathrm{Fe}]$ & 0.09 & 0.04 & 0.22 & 35 & -0.05 & 0.03 & 0.12 & 21 & 0.31 & 0.04 & 0.13 & 14 \\
\hline$[\mathrm{La} / \mathrm{Fe}]$ & 0.14 & 0.03 & 0.18 & 34 & 0.01 & 0.01 & 0.06 & 20 & 0.33 & 0.02 & 0.09 & 14 \\
\hline$[\mathrm{Nd} / \mathrm{Fe}]$ & 0.17 & 0.03 & 0.19 & 32 & 0.05 & 0.02 & 0.09 & 20 & 0.37 & 0.03 & 0.11 & 12 \\
\hline$[\mathrm{Eu} / \mathrm{Fe}]$ & 0.46 & 0.01 & 0.07 & 30 & 0.49 & 0.01 & 0.05 & 19 & 0.42 & 0.02 & 0.08 & 11 \\
\hline$[\mathrm{La} / \mathrm{Y}]$ & 0.06 & 0.03 & 0.15 & 31 & 0.11 & 0.04 & 0.16 & 19 & -0.01 & 0.03 & 0.11 & 12 \\
\hline$[(\mathrm{C}+\mathrm{N}+\mathrm{O}) / \mathrm{Fe}]$ & 0.33 & 0.02 & 0.08 & 14 & 0.28 & 0.02 & 0.05 & 8 & 0.41 & 0.02 & 0.04 & 6 \\
\hline $\log \epsilon(\mathrm{C}+\mathrm{N}+\mathrm{O})$ & 7.68 & 0.04 & 0.16 & 14 & 7.57 & 0.03 & 0.09 & 8 & 7.84 & 0.03 & 0.07 & 6 \\
\hline
\end{tabular}

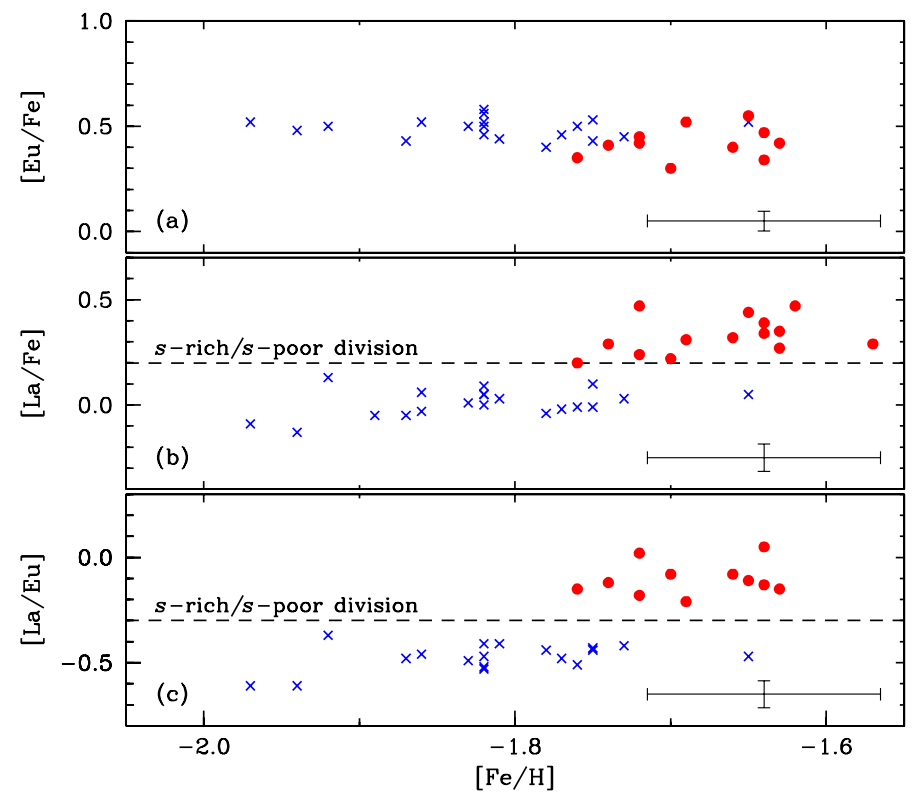

Fig. 7. La and $\mathrm{Eu}$ abundances of $\mathrm{M} 22$ as functions of $[\mathrm{Fe} / \mathrm{H}]$. In panels a) and b) we repeat the $[\mathrm{Eu} / \mathrm{Fe}]$ and $[\mathrm{La} / \mathrm{Fe}]$ panels of Fig. 6. In panel b) we have added a dashed line at $[\mathrm{La} / \mathrm{Fe}]=+0.2$ to show the division in this abundance ratio between $s$-rich and $s$-poor stars; see text for discussion of this choice. In panel c) we plot the [La/Eu] values; the separation between the two groups of stars is more obvious here, and the dashed line represents our chosen split at $[\mathrm{La} / \mathrm{Eu}]=-0.3$. Symbols are as in Fig. 6.

might indicate that there is no $[\mathrm{Fe} / \mathrm{H}]$ spread within each group, if the relative errors are of the order of 0.05 dex. Some small overlap in metallicity between the two $s$ (and Fe)-groups, at roughly $-1.8 \lesssim[\mathrm{Fe} / \mathrm{H}] \lesssim-1.7$ cannot be excluded, as apparent

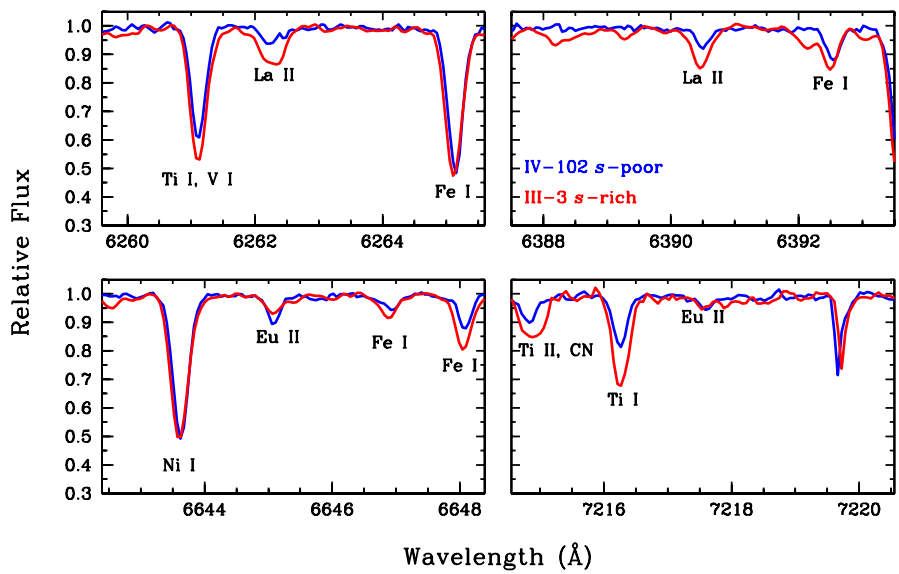

Fig. 8. Comparison of the spectra of La lines (top panels) and Eu lines (bottom panels) in two stars with similar atmospheric parameters but substantially different derived La abundances. The displayed data are taken from the MCD spectra. The spectrum in red is that of the $s$-rich star III-3, and the one in blue is that of the s-poor star IV- 102 .

in the top panel of Fig. 10, but the difference in $[\mathrm{Fe} / \mathrm{H}]$ between $s$-rich and $s$-poor groups is not much larger than the estimated observational error associated to $[\mathrm{Fe} / \mathrm{H}]$ of $\sim 0.07$.

As demonstrated in M09 (see their Fig. 12), due to the limits imposed by our observational errors, the relatively small difference in $[\mathrm{Fe} / \mathrm{H}]$ between the two stellar groups in M 22 is much more clearly recognizable by separating stars on the basis of their $s$-richness. We note however that a similar difference in $[\mathrm{Fe} / \mathrm{H}]$ as the one found in M09 and confirmed here, is also confirmed by DC09 who found that two groups of stars with mean metallicities of $[\mathrm{Fe} / \mathrm{H}]-1.63$ and $[\mathrm{Fe} / \mathrm{H}]=-1.89$. 
A. F. Marino et al.: The two metallicity groups in M 22
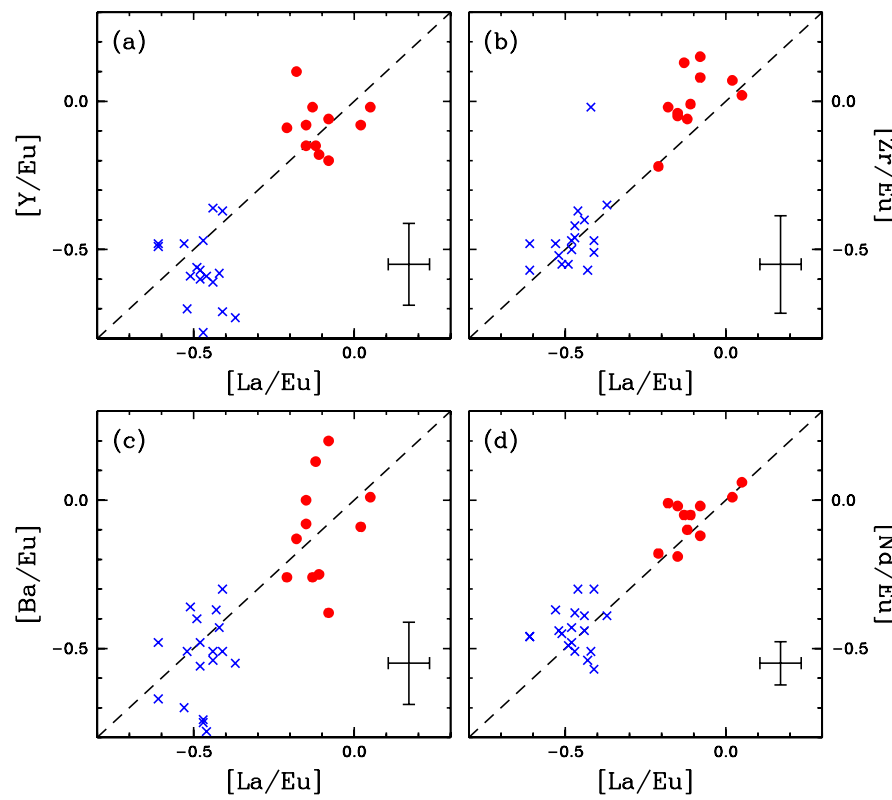

Fig. 9. Abundance ratios $[\mathrm{X} / \mathrm{Eu}]$ for elements $\mathrm{Y}, \mathrm{Zr}, \mathrm{Ba}$, and $\mathrm{Nd}$ as functions of $[\mathrm{La} / \mathrm{Eu}]$. In each panel, the dashed line represents equality of the displayed abundance ratios. Symbols are as in Fig. 6.
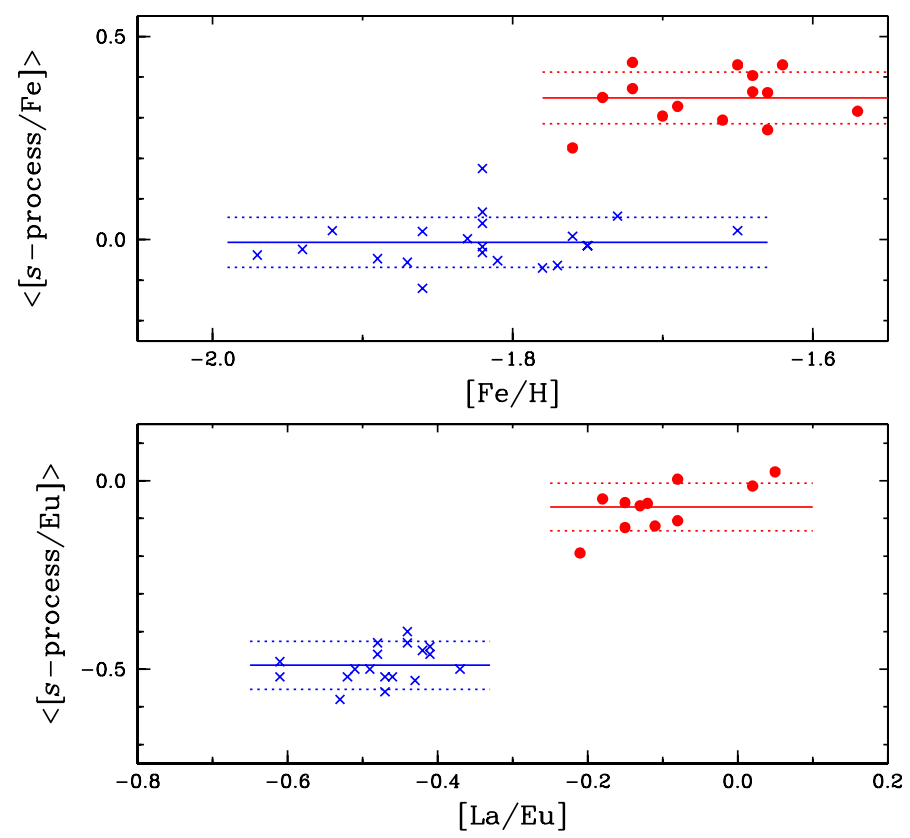

Fig. 10. Upper panel: average $s$-process abundance ratios with respect to $\mathrm{Fe}$, plotted as functions of $[\mathrm{Fe} / \mathrm{H}]$. Bottom panel: average $s$-process abundance ratios with respect to Eu, plotted as functions of [La/Eu]. See the text for how the averages were computed. In each panel, the solid lines represent the means for each group, and the dotted lines represent the sample deviations $\sigma$. Symbols are as in Fig. 6.

Our focus in the rest of the present paper is to study the abundance behavior of different elements in stars belonging to the $s$-poor or $s$-rich groups, as defined by their $[\mathrm{La} / \mathrm{Eu}]$ ratios $^{6}$.

\footnotetext{
6 As is often the case with shorthand labels, the $s$-rich and $s$-poor designations should not be taken too literally. In M 22, the s-poor population alternatively could have been labeled " $r$-rich", because they have $[s$-process $/ \mathrm{Fe}] \simeq 0.0$ and $[r$-process $/ \mathrm{Fe}] \simeq+0.4$. The $s$-rich population could have been labeled " $r+s$-rich" because they have overabundances of all $n$-capture elements relative to Fe. What is secure is the addition of
}
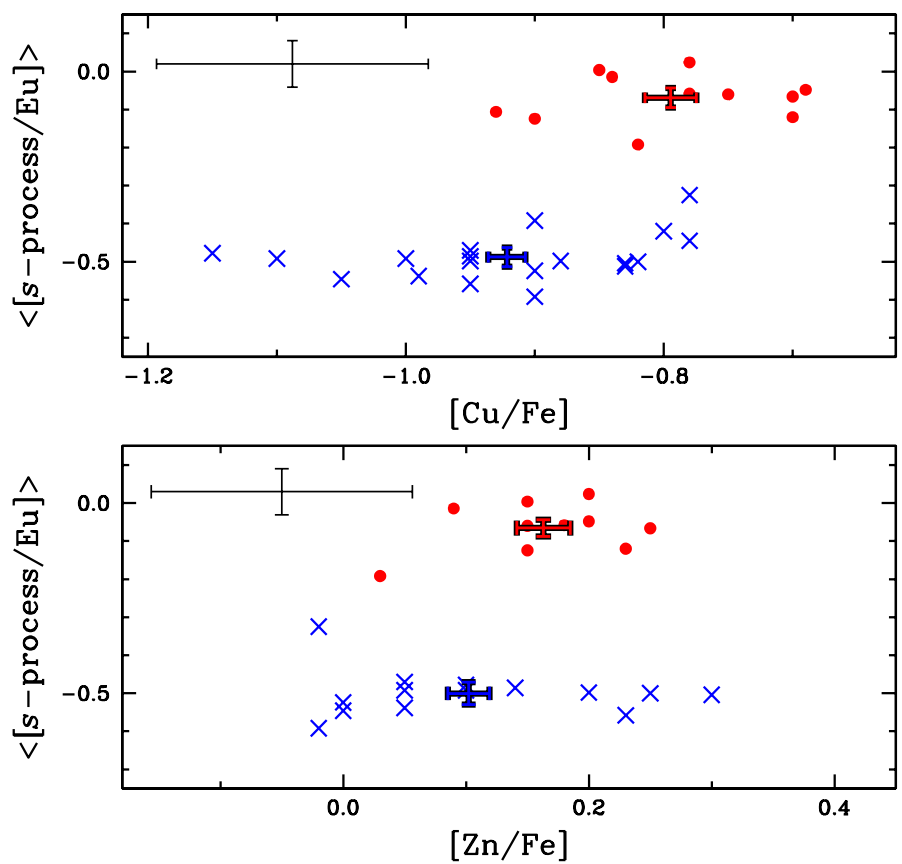

Fig. 11. Average $s$-process abundance ratios with respect to Eu, plotted as functions of the ratios of the heavy Fe-peak elements $\mathrm{Cu}$ and $\mathrm{Zn}$ to Fe. Error bars in blue and red represent the mean values for the $s$-rich and $s$-poor stars. Symbols are as in Fig. 6.

\subsection{The heavy Fe-peak elements}

Copper is very underabundant in M22, just as it is in other low metallicity field stars (Sneden et al. 1991; Mishenina et al. 2002) and globular clusters (Simmerer et al. 2003). However, $[\mathrm{Cu} / \mathrm{Fe}]$ appears to vary in concert with the $s$-process elements, being higher in the $s$-rich than the $s$-poor group by $\Delta_{\text {poor }}^{\text {rich }}[\mathrm{Cu} / \mathrm{Fe}]=$ $+0.15 \pm 0.04$ (Table 7). In the top panel of Fig. 11 we illustrate the $\mathrm{Cu}$ distributions in the two groups, plotting them versus their $s$-process enrichment. The much larger spread in individual $[\mathrm{Cu} / \mathrm{Fe}]$ values in the $s$-poor stars compared to the $s$-rich stars is worth noting. For each $s$-group we represented the mean $\mathrm{Cu}$ abundance and the associated rms. The difference among the two groups is at $3 \sigma$ level. However, given the uncertainties associated with individual $\mathrm{Cu}$ abundance measurements, interpretation of this trend should be viewed with caution. Certainly the $s$-rich $/ s$-poor $[\mathrm{Cu} / \mathrm{Fe}]$ difference is much less than that observed for the $s$-process elements discussed in Sect. 5.1.

If the $[\mathrm{Cu} / \mathrm{Fe}]$ trend is real, it could put some new constraints on scenarios for the origin of this element. The nucleosynthetic sites of copper have been discussed by Sneden et al. (1991), who suggested that much of the $\mathrm{Cu}$ in metal-poor stars forms in the weak component of the $s$-process, at which time neutron captures on Fe-peak elements take place during the late stages of core He-burning (Couch et al. 1974; Raiteri et al. 1991) in massive stars. Because the weak component of the $s$-process is a secondary mechanism for nucleosynthesis, this site for the formation of copper agrees qualitatively with a relationship of increasing $[\mathrm{Cu} / \mathrm{Fe}]$ as a function of $[\mathrm{Fe} / \mathrm{H}]$ in metal-poor stars. The $\mathrm{Cu}$ distribution that we observe in M 22 provides mild support for such an $s$-process origin, due to the weak component in massive stars, for the more metal-rich stars.

much more $s$-process than $r$-process material in the higher metallicity stars of M 22. 
As a check on the $\mathrm{Cu}$ results, note that our $\mathrm{Zn}$ abundances exhibit no trend with $s$-process enrichment within the observational errors: $\Delta_{\text {poor }}^{\text {rich }}[\mathrm{Zn} / \mathrm{Fe}]=+0.06 \pm 0.04$ (Table 7). The individual abundances are plotted versus $s$-process enrichment in the bottom panel of Fig. 11. The $s$-poor and $s$-rich stars occupy the same $[\mathrm{Zn} / \mathrm{Fe}]$ space in this figure. No observable difference in $\mathrm{Zn}$ between $s$-rich and $s$-poor groups would have been expected from theory. However the observational errors (represented by error bars in Fig. 11) are large enough to mask possible small abundance variations within the two groups. The small positive value of $\delta[\mathrm{Zn} / \mathrm{Fe}]$ probably illuminates the limit of our abundance set to provide meaningful nucleosynthesis scenarios for M 22.

\subsection{The $\alpha$ elements}

The observed $\alpha$ elements in $\mathrm{M} 22$ are $\mathrm{Si}, \mathrm{Ca}$, and $\mathrm{Ti}$. We do not include $\mathrm{O}$ and $\mathrm{Mg}$ in this group because in globular clusters their abundances can be affected by proton capture nucleosynthesis, i.e. different stars in GCs can have lower O, and in some cases lower Mg. All $\alpha$ elements are overabundant in M 22. From the data in Table 7 for the whole 35-star sample, we obtain $\langle[\alpha / \mathrm{Fe}]\rangle=+0.33 \pm 0.01(\sigma=0.04)$. These $\alpha$-element enhancements are in excellent agreement with those reported by M09. Note that the mean $\mathrm{O}$ and $\mathrm{Mg}$ abundances are nearly the same as the other $\alpha$ 's: $\langle[\mathrm{O} / \mathrm{Fe}]\rangle=+0.34$ and $\langle[\mathrm{Mg} / \mathrm{Fe}]\rangle=+0.39$. These elements will be discussed in detail in Sect. 5.4.

No correlation with $s$-process peculiarity can be detected for $\mathrm{Si}$, and $\mathrm{Ti}$; their $[\mathrm{X} / \mathrm{Fe}]$ ratios are identical in $s$-rich and $s$-poor stars, within the abundance measurement uncertainties. The Ti I-Ti II abundance differences have a small trend with temperature, due almost entirely to a variation in $\mathrm{Ti}$ I; the Ti II abundance distribution is constant with $T_{\text {eff }}$. This behavior may reflect NLTE effects in the Ti I abundances. NLTE overionization of Ti I is likely to be occurring, for the average Ti II abundances are higher than Ti i ones. Probably Ti in abundances are more reliable than Ti i ones (see Bergemann 2011, for a detailed discussion).

From UVES spectra of 17 stars, M09 claimed that a small but statistically significant positive correlation existed between $[\mathrm{Ca} / \mathrm{Fe}]$ and $[\mathrm{Fe} / \mathrm{H}]$ (or $[s$-process/Fe]). As shown in Fig. 12, our larger sample confirms this trend at approximately the same level: $\Delta_{\text {poor }}^{\text {rich }}[\mathrm{Ca} / \mathrm{Fe}]=+0.10 \pm 0.02$ (Table 7).

\subsection{The proton-capture elements}

We now consider the abundances for $\mathrm{C}, \mathrm{N}, \mathrm{O}, \mathrm{Na}, \mathrm{Mg}$, and $\mathrm{Al}$, all of which can be affected by proton capture reactions. In Fig. 13 we display the $[\mathrm{X} / \mathrm{Fe}]$ values obtained for each of these elements as a function of the mean $\langle[s$-process $/ E u]\rangle$ abundance. As in previous figures, we use different symbols to represent the $s$-poor and $s$-rich groups. The means and the $\sigma$ values obtained for each group are also shown. Since for 5 out 8 stars in the $s$-poor groups we were able to measure only upper limits for the $\mathrm{N}$ abundance, the plotted mean is of course also an upper limit.

Inspection of Fig. 13 suggests that $s$-rich stars have, on average, higher $\mathrm{C}$ and $\mathrm{N}$ contents, and from Table 7 we compute $\Delta_{\text {poor }}^{\text {rich }}[\mathrm{C} / \mathrm{Fe}]=+0.35 \pm 0.13$ and $\Delta_{\text {poor }}^{\text {rich }}[\mathrm{N} / \mathrm{Fe}]=+0.47 \pm 0.11$. As noted by M09, correlations (with large star-to-star scatter) are observed between the $s$-process groups with $\mathrm{Na}$ and $\mathrm{Al}$ : $\Delta_{\text {poor }}^{\text {rich }}[\mathrm{Na} / \mathrm{Fe}]=+0.23 \pm 0.07$ and $\Delta_{\text {poor }}^{\text {rich }}[\mathrm{Al} / \mathrm{Fe}]=+0.21 \pm 0.10$. In contrast, the $p$-capture $\mathrm{O}$ and $\mathrm{Mg}$ elements have nearly the same abundances in both groups: $\Delta_{\text {poor }}^{\text {rich }}[\mathrm{O} / \mathrm{Fe}]=-0.04 \pm 0.07$ and $\Delta_{\text {poor }}^{\text {rich }}[\mathrm{Mg}]=+0.01 \pm 0.04$.

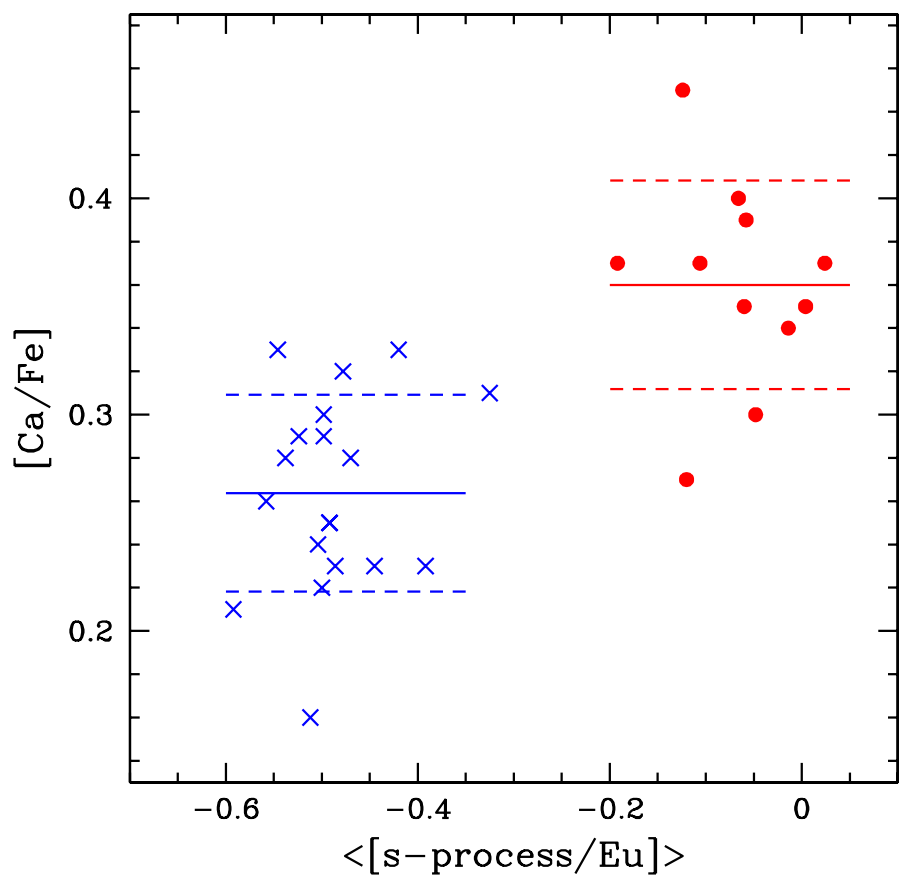

Fig. 12. Calcium abundance ratios plotted as a function of the average $s$-process abundance ratios with respect to Eu. Symbols are as in Fig. 6.

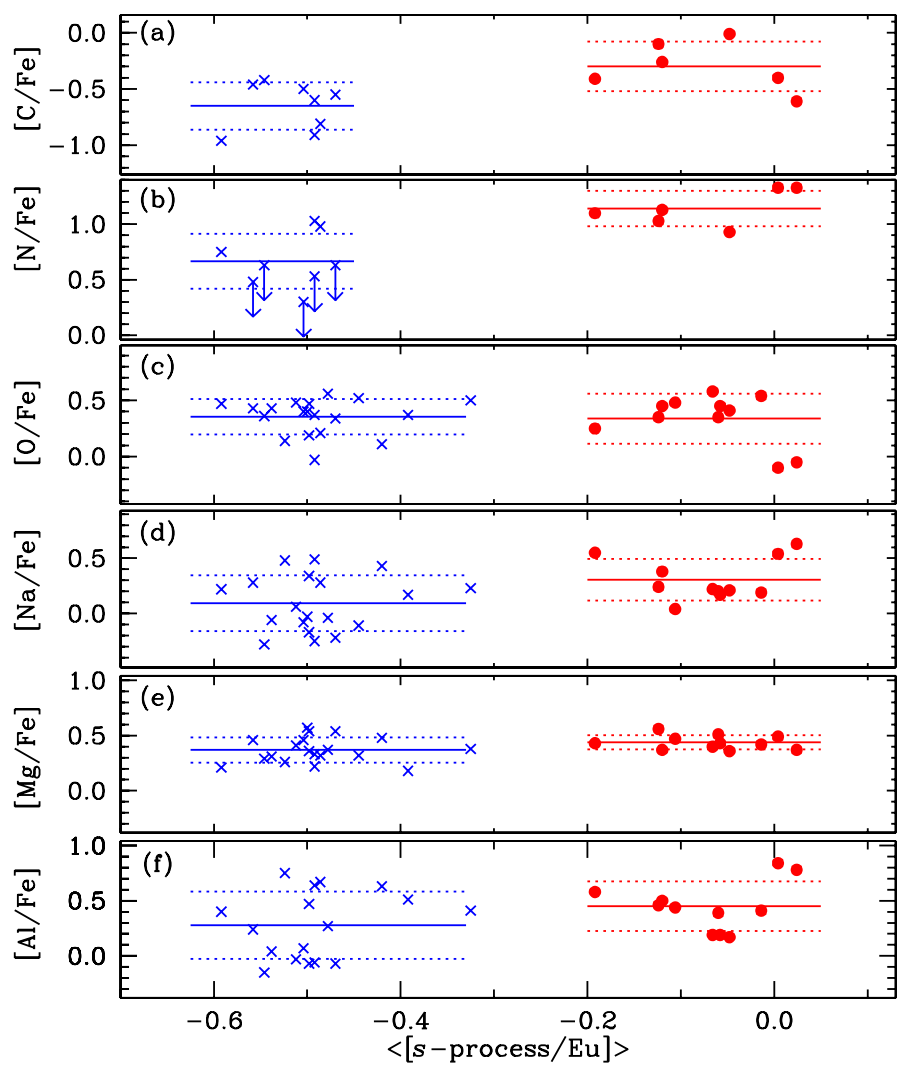

Fig. 13. $[\mathrm{C}, \mathrm{N}, \mathrm{O}, \mathrm{Na}, \mathrm{Mg}, \mathrm{Al} / \mathrm{Fe}]$ abundance ratios as a function of the mean [s-process/Eu] content. Symbols are as in Fig. 6.

More illuminating are the abundance comparisons among the $p$-capture elements. As shown by M09, Na and $\mathrm{O}$ abundances are anticorrelated in M 22 (see their Fig. 3) just as they are in all GCs that have been studied so far. In Fig. 14 we show the $\mathrm{Na}$ and $\mathrm{O}$ data for our larger sample, by using both the LTE $\mathrm{Na}$ values (left lower panel) and the NLTE ones (right lower 

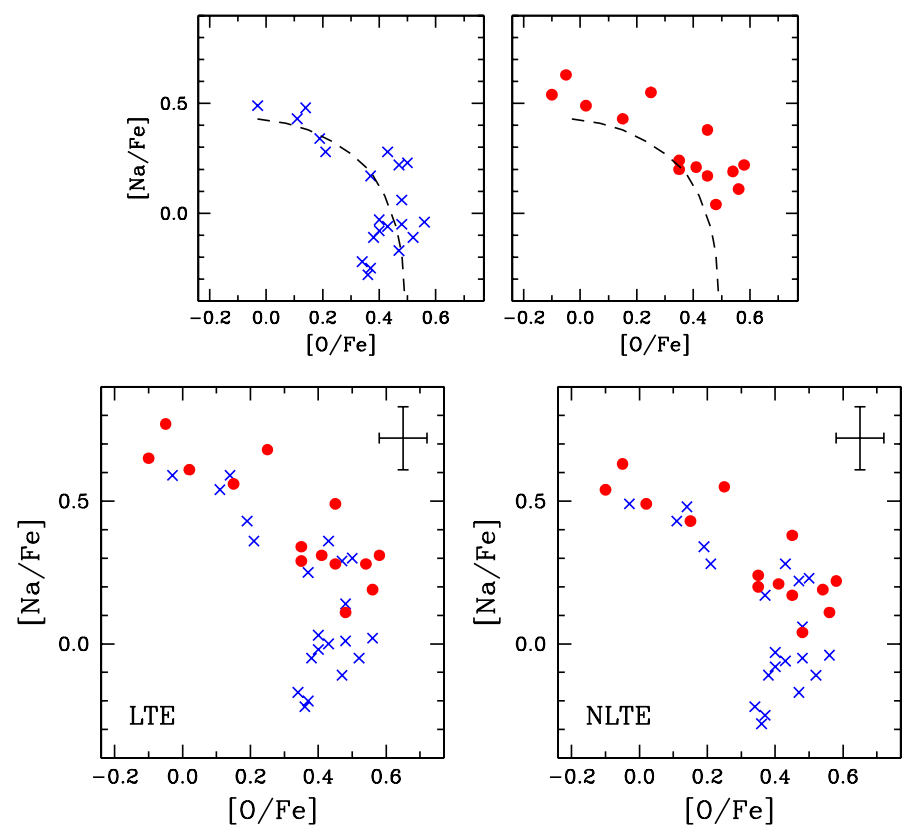

Fig. 14. $[\mathrm{Na} / \mathrm{Fe}]$ as a function of $[\mathrm{O} / \mathrm{Fe}]$. The bottom panels display all stars in the Na-O plane by using LTE (left) and NLTE Na abundances (right). The top left-hand panel contains only s-poor stars and the top right-hand panel contains only s-rich stars. The dashed lines in the top panels represent a free-hand representation of the mean $\mathrm{Na}-\mathrm{O}$ trend of only the $s$-poor stars. Symbols are as in Fig. 6.
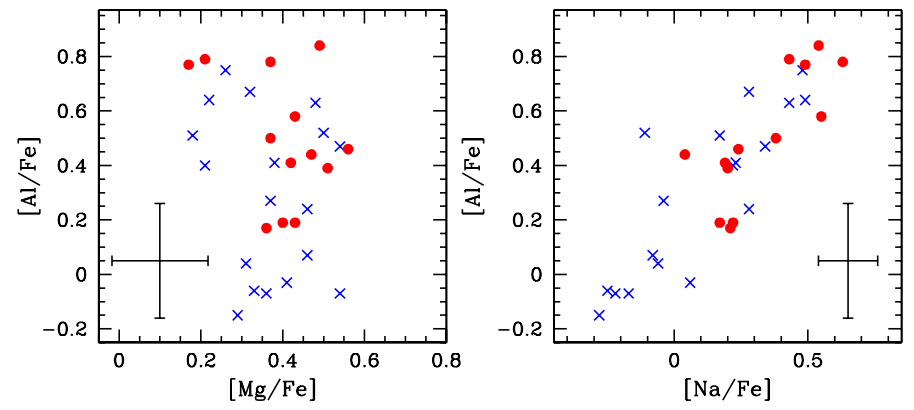

Fig. 15. $[\mathrm{Al} / \mathrm{Fe}]$ abundance ratios as a function of $[\mathrm{Na} / \mathrm{Fe}]$ (left panel), and $[\mathrm{Mg} / \mathrm{Fe}]$ (right panel). Symbols are as in Fig. 6.

panel). Our sample makes it clear that the $\mathrm{NaO}$ anticorrelation exists in both M 22 populations, but in somewhat different domains of O-Na space. The $s$-rich and $s$-poor stars span a similar range in oxygen, $-0.1 \lesssim[\mathrm{O} / \mathrm{Fe}] \lesssim+0.6$, but not in sodium: $-0.3 \lesssim[\mathrm{Na} / \mathrm{Fe}]_{s \text {-poor }} \lesssim+0.5$ and $+0.0 \lesssim[\mathrm{Na} / \mathrm{Fe}]_{s \text {-rich }} \lesssim+0.6$. To better visualize the difference between the $\mathrm{Na}-\mathrm{O}$ pattern in the two $s$-process groups, we have drawn by hand a fiducial line tracing the Na-O anticorrelation shape for $s$-poor stars, and superimpose this line to the $s$-rich stars (upper panels of Fig. 14). With this aid, we estimate that at any $[\mathrm{O} / \mathrm{Fe}]$ ratio, the $[\mathrm{Na} / \mathrm{Fe}]$ ratio in an $s$-rich star is $\sim 0.2$ dex larger than in an $s$-poor star. The different behavior of $s$-poor and $s$-rich stars in the Na-O anticorrelation, even if with some differences, recalls the one in $\omega$ Cen (see Johnson \& Pilachowski 2010; Marino et al. 2011).

Both M 22 populations exhibit positive abundance correlations of $\mathrm{Al}$ with $\mathrm{Na}$, as illustrated in the right panel of Fig. 15. However, here also the $s$-poor and $s$-rich stars are distinguishable: only the $s$-poor stars have $[\mathrm{Na}$ or $\mathrm{Al} / \mathrm{Fe}]<0$. No $\mathrm{Mg}$ variation with either $\mathrm{Al}$ or $\mathrm{Na}$ were discovered by $\mathrm{M} 09$, and our expanded sample confirms this result (see the left panel of Fig. 15). M09 suggested that an intrinsic Mg variation might be too small to be detected in the face of observational errors. For our complete 35 -star sample, as well as the $s$-poor and $s$-rich subsamples, $\sigma[\mathrm{Mg} / \mathrm{Fe}] \simeq 0.11-0.12$ (Table 7). Such values are not much larger than those for $[\mathrm{Si} / \mathrm{Fe}],[\mathrm{Ca} / \mathrm{Fe}],[\mathrm{Ti} / \mathrm{Fe}]$, and $[\mathrm{Zn} / \mathrm{Fe}]$, which we claim are invariant in M 22 stars. A possible $\mathrm{Mg}$-Al anticorrelation may be inferred from the half-dozen stars in Fig. 15 with the lowest $[\mathrm{Mg} / \mathrm{Fe}]$ ratios; these all have high $[\mathrm{Al} / \mathrm{Fe}]$ ratios. However, this is not a statistically defensible conclusion, and pursuit of this point would need careful analysis of a larger M 22 sample.

The lack of a clear $\mathrm{Mg}$ - $\mathrm{Al}$ anticorrelation does not necessarily mean that proton captures on $\mathrm{Mg}$ are ruled out. If we suppose that the higher observed $\mathrm{Mg}$ abundances are representative of "primordial" M22 material (that is, prior to any $p$-capture synthesis events), and if primordial $\mathrm{Al}$ is indicated by the lower observed $\mathrm{Al}$ abundances, then for this material $[\mathrm{Mg} / \mathrm{Al}] \sim+0.5$, or $\log \epsilon(\mathrm{Mg} / \mathrm{Al}) \sim+1.6$. Then if (for example) $10 \%$ of this $\mathrm{Mg}$ were to be converted to ${ }^{27} \mathrm{Al}$ by $p$-capture in the primordial material, the resulting $\mathrm{Al}$ would go up by a factor of four, nearly the range covered by our data. The $10 \%$ decrease in $\mathrm{Mg}$ would be nearly impossible to be detected. Additionally, if the ab initio abundance of $\mathrm{Mg}$ contains substantial amounts of ${ }^{25} \mathrm{Mg}$ and/or ${ }^{26} \mathrm{Mg}$, then the final $\mathrm{Al}$ abundance would be even larger after p-captures.

In Fig. 16 we present correlations between $\mathrm{C}$ abundances and $\mathrm{N}, \mathrm{Na}, \mathrm{O}$ abundances. No trends among these elements are apparent if we consider our sample of stars as a whole. However, segregation of points into two different $s$-Fe groups provides evidence for unique C-N (left-hand panel) and C-Na (middle panel) anticorrelations within each group, as expected from CNO-cycle enrichment within each M 22 population separately. However, we cannot discern any obvious nucleosynthetic signature in the $\mathrm{C}-\mathrm{O}$ plot (right-hand panel), either from the whole sample or in the two populations individually.

In Fig. 17 the CNO abundance sum is represented as a function of $[s / \mathrm{Fe}]$, as usual calling the reader's attention to the $s$-poor and $s$-rich groups with different symbols in the figure. These CNO totals are shown as $[(\mathrm{C}+\mathrm{N}+\mathrm{O}) / \mathrm{Fe}]$ in the lefthand panel and as $\log \epsilon(\mathrm{C}+\mathrm{N}+\mathrm{O})$ in the right-hand panel. The M 22 population split is evident: $s$-rich stars have on average a higher $[(\mathrm{C}+\mathrm{N}+\mathrm{O}) / \mathrm{Fe}]$ abundance, with $\Delta_{\text {poor }}^{\text {rich }}[(\mathrm{C}+\mathrm{N}+\mathrm{O}) / \mathrm{Fe}]=$ $+0.13 \pm 0.03$. The mean difference in the $[(\mathrm{C}+\mathrm{N}+\mathrm{O}) / \mathrm{Fe}]$ content is at the level of $\gtrsim 3 \sigma$ (see Table 7), but note that we could measure only an upper limit to the $\mathrm{N}$ abundance for almost all the $s$-poor stars, suggesting that the real difference could be larger.

A final piece of evidence in the M22 abundance puzzle comes from our analysis of the $8000 \AA$ region of six MCD stars for which we were able to derive ${ }^{12} \mathrm{C} /{ }^{13} \mathrm{C}$ ratios (Table 5). Note that star IV-102 has very weak $\mathrm{CN}$ red-system bands at all wavelengths, and our derived ${ }^{12} \mathrm{C} /{ }^{13} \mathrm{C}$ value should be treated with caution. The APO and LICK spectra also cover the $8000 \AA$ spectral region. However, neither of these data sets have sufficient resolving power and $S / N$ to permit reliable carbon isotopic ratios.

Although the six stars with derived carbon isotopic ratios constitute only a small subset of our M22 giants, all of them have $3.0<{ }^{12} \mathrm{C} /{ }^{13} \mathrm{C}<5.0$ (Table 5). Our low value for III-3 is supported by the earlier Brown et al. (1990) analysis of this star, for which they obtained ${ }^{12} \mathrm{C} /{ }^{13} \mathrm{C}=4$. Star IV-20, with no $\mathrm{MCD}$ data in the present study, also has a very low isotopic ratio according to Brown et al. : ${ }^{12} \mathrm{C} /{ }^{13} \mathrm{C}=4$. Finally, Smith \& Suntzeff (1989) used low-resolution spectra of the CO infrared first-overtone vibration-rotation bands to derive ${ }^{12} \mathrm{C} /{ }^{13} \mathrm{C}$ estimates for five M 22 stars. For three stars (III-3, III-12, and IV- 

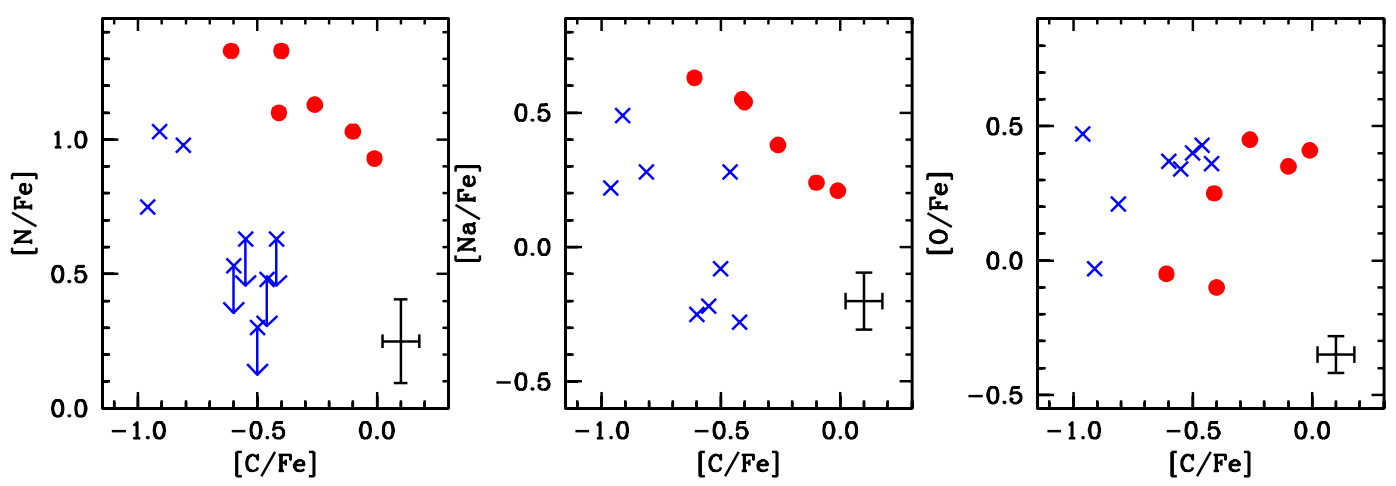

Fig. 16. From the left to the right: nitrogen, sodium and oxygen abundance ratios as a function of [C/Fe]. Symbols are as in Fig. 6.
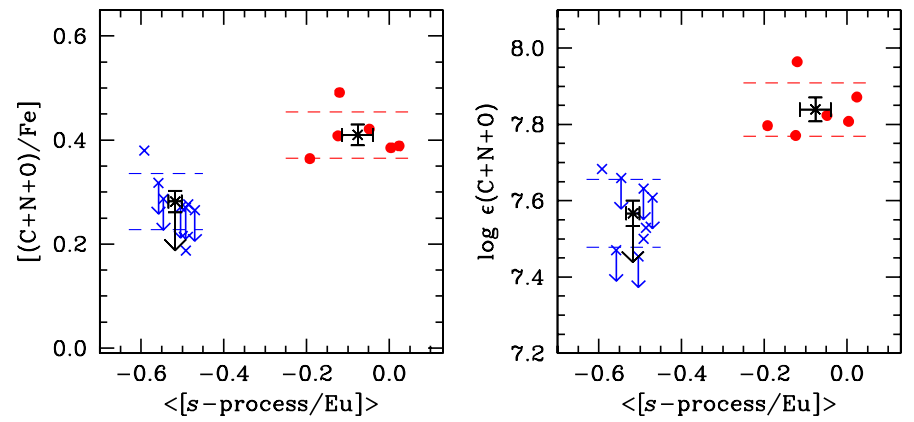

Fig. 17. $[(\mathrm{C}+\mathrm{N}+\mathrm{O}) / \mathrm{Fe}]$ (left panel) and $\log \epsilon(\mathrm{C}+\mathrm{N}+\mathrm{O})$ (right panel) as a function of $\langle[s$-process/Eu $]\rangle$. Symbols are as in Fig. 6 . The dashed lines represent the error associated with single measures, the black error bars represent the mean $\mathrm{CNO}$ abundance contents for the two $s$-groups and the statistical error associated with the mean.

102) their ratios are in excellent agreement with ours. However, for stars IV-97 and V8 (with no available values in our study) Smith \& Suntzeff derived higher ${ }^{12} \mathrm{C} /{ }^{13} \mathrm{C}$ values: $\geq 10$ and $\geq 40$, respectively. In summary, the carbon isotopic values for most M 22 giants appear to be very low, normal for the stars similar to the ones studied here, but a larger-sample study will be needed to determine if stars with substantially larger ratios are few in number or common.

\section{M22 low resolution spectroscopy and photometry}

Norris \& Freeman (1983) gathered low resolution blue spectra of 130 giants in M 22, and from these data determined three absorption indices: $S$ (3839), for the CN 3883 bandhead strength; $A(\mathrm{Ca})$, for the $\mathrm{Ca}$ II $\mathrm{H} \& \mathrm{~K}$ strength; and $W(\mathrm{G})$, for the $\mathrm{CH} \mathrm{G}-$ band strength. The $\mathrm{CN}$ and the $\mathrm{Ca}$ indices were corrected to first order for the natural changes in absorption strengths due to $T_{\text {eff }}$ and $\log g$ differences along the M 22 giant branch. This was accomplished by first drawing fiducial lines to express the strength changes as functions of $V$ magnitude, and then measuring offsets $\delta S(3839)$ and $\delta A(\mathrm{Ca})$ from these lines. Norris \& Freeman showed that a positive correlation exists between $\delta S(3839)$ and $\delta A(\mathrm{Ca})$, as well as between $W(\mathrm{G})$ and $\delta A(\mathrm{Ca})$. In Fig. 18 we compare these indices with some abundances derived in this paper. The upper two panels, showing the $S(3839)$ index as a function of $[\mathrm{Fe} / \mathrm{H}]$ and $[\mathrm{La} / \mathrm{Eu}]$, clearly demonstrate that our derived $[\mathrm{Fe} / \mathrm{H}]$ metallicities and the $s$-process abundances also track the $\mathrm{CN}$ strength indices. In the middle panels we show that there is a mid correlation between $W(\mathrm{G})$ with $[\mathrm{C} / \mathrm{Fe}]$ and $S(3839)$ with $[\mathrm{N} / \mathrm{Fe}]$. Note also the positive correlation between the $A(\mathrm{Ca})$ in-
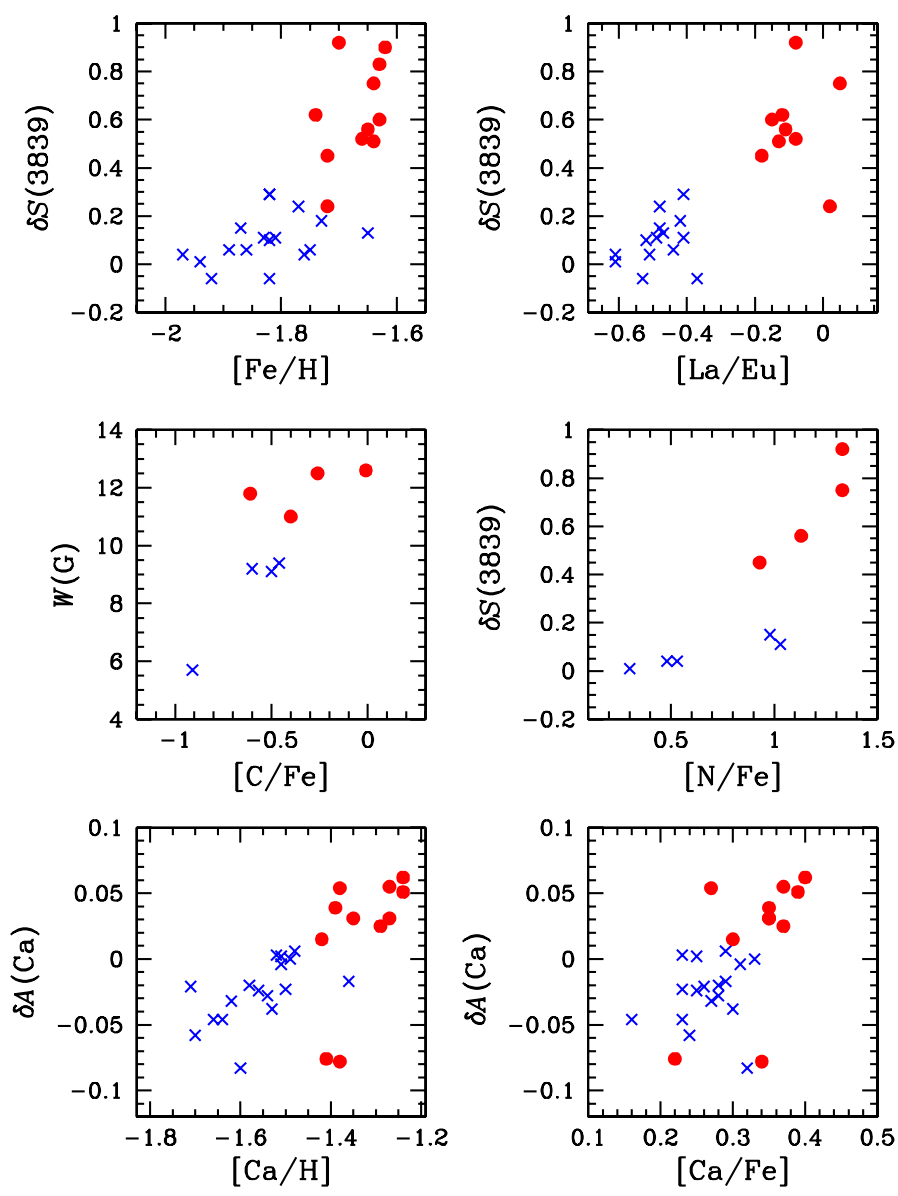

Fig. 18. Comparison of the chemical abundances derived in this paper with the indices by Norris \& Freeman (1983): CN band-strength indices $\delta S(3839)$ plotted as a function of the $[\mathrm{Fe} / \mathrm{H}]$ (left-upper panel) and $[\mathrm{La} / \mathrm{Eu}]$ (right-upper panel), $W(\mathrm{G})$ and $\delta S(3839)$ as a function of $[\mathrm{C} / \mathrm{Fe}]$ (left-middle panel) and $[\mathrm{N} / \mathrm{Fe}]$ (right-middle panel), and $\delta A(\mathrm{Ca})$ as a function of $[\mathrm{Ca} / \mathrm{H}]$ (left-bottom panel) and $[\mathrm{Ca} / \mathrm{Fe}]$ (right-bottom panel). Symbols are as in Fig. 6.

dex and our derived abundance ratios $[\mathrm{Ca} / \mathrm{H}]$ and $[\mathrm{Ca} / \mathrm{Fe}]$ (lower two panels).

In addition we have coupled our abundances to the Strömgren photometric data of Richter et al. (1999). Those authors demonstrated the existence of a bimodal distribution in the $m_{1}$ index of M 22 giants, which they associated with $\mathrm{CN}$ variations. Here we match our spectroscopic results with photometry in two different ways. In the top panels of Fig. 19 purely photometric data are displayed, combining the Strömgren colours with 
A. F. Marino et al.: The two metallicity groups in M 22

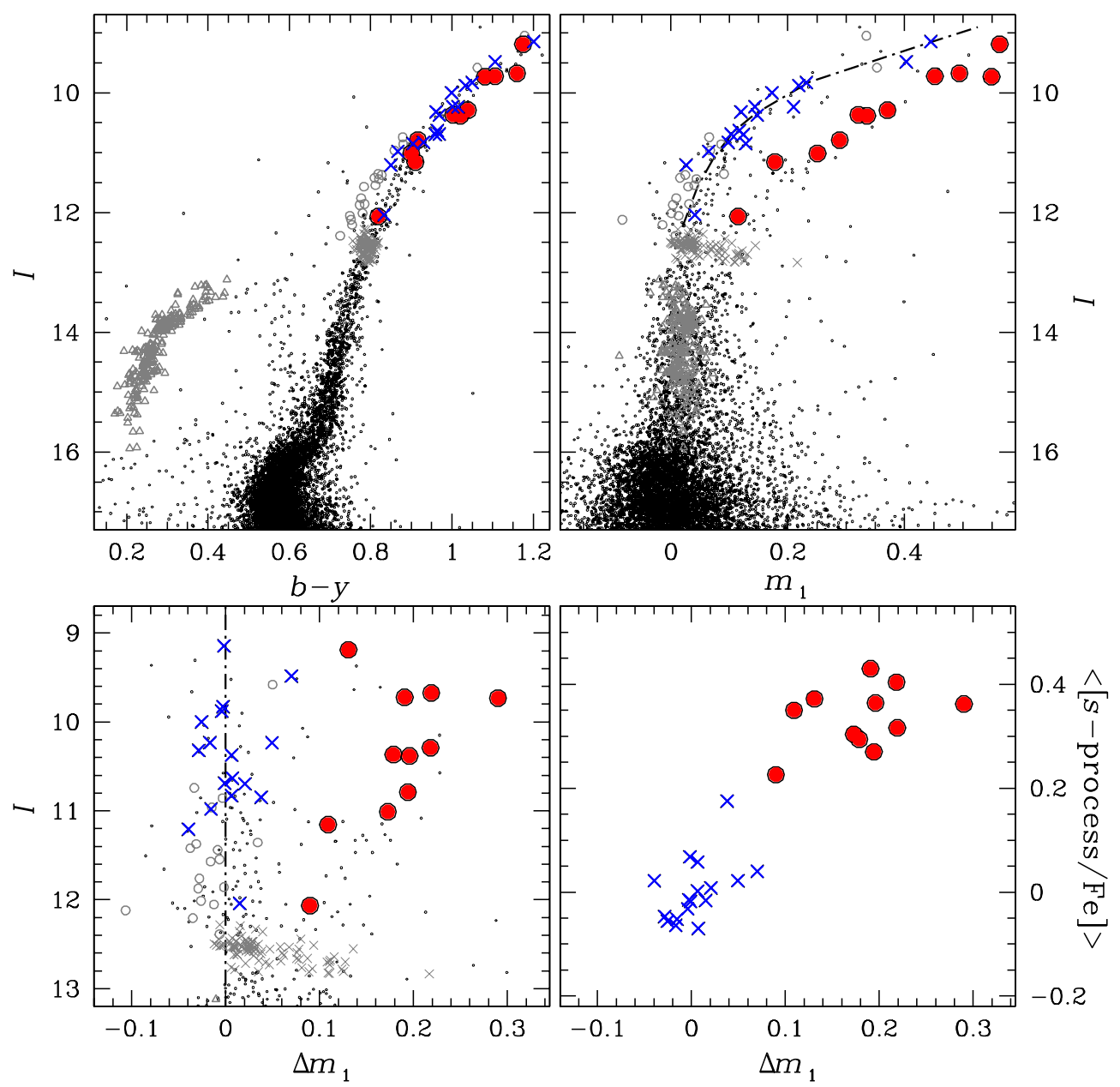

Fig. 19. Top panels: $I-b-y$ (left) and $I-m_{1}$ (right) CMD for M 22, corrected for differential reddening. The grey symbols represent HB stars (triangles), probable AGB stars (circles) and RGB bump stars (crosses) selected in the $I-(b-y)$ CMD. Spectroscopic data are superimposed with $s$-rich stars represented by red circles, and $s$-poor stars by blue crosses, according with the other figures. Bottom panels: colour difference $\Delta m_{1}$ between each analyzed star and a reference fiducial line represented as a dotted line (left panel). On the right panel the $\langle[s / \mathrm{Fe}]\rangle$ average abundances are shown as a function of $\Delta m_{1}$. The sources for the photometric data are given in Sect. 2.

I magnitudes taken from Stetson's database, after correcting the data for differential reddening. Our stars, coded as in previous figures, show little differences in the $b-y$ versus $I$ plot (top-left panel). However, a clear split is apparent between the $s(\mathrm{Fe})$-poor and $s(\mathrm{Fe})$-rich stars in $m_{1}$ versus $I$ plot (top-right panel). As the $m_{1}$ index is strongly affected by the blue $\mathrm{CN}$ bands and overall metallicity, we expected this bimodal distribution as a consequence of the higher mean abundance in both $\mathrm{C}$ and $\mathrm{N}$ of the $s$-rich stars, as shown in Fig. 13. Hence, the $s$-rich stars populate the RGB sequence associated to the stars enriched in $\mathrm{CN}$, and $s$-poor stars the branch associated to weaker $\mathrm{CN}$ band strengths (see Richter et al. 1999).

Of course, our sample of stars could be contaminated by AGB stars, but the presence of few AGB stars does not affect the results, as discussed also in M09. A visual inspection of the stars on the CMD on the right-upper panel of Fig. 19, suggests that some AGB stars could be present among both the $s$-poor and the $s$-rich RGB. The AGB lies blueward of the RGB at a given luminosity. But identification of blue-offset stars in the $I$ vs. $b-y$ CMD (left-upper panel of Fig. 19) as probable members of the AGB could be misleading, because we also expect to find the slightly more metal-poor, $s$-poor stars on the blue side of the RGB. Unambiguous assignment of AGB stars in this manner is not easy.
On the other hand, systematically redder colours for the $s$-rich stars would lead to a low probability of being shifted by photometric errors into the AGB region. Indeed in M09, six out of seven probable AGB stars, belong to the $s$-poor group. Note that the possible presence of AGB stars in M09 was based only on a visual inspection of the stars in the $I$ vs. $(B-I) \mathrm{CMD}$, and doesn't necessarily mean that AGB stars preferentially belong to the $s$-poor group.

In any case, assuming that all the probable AGB stars in M09 are indeed true AGB members, their $s$-poor sample would include half RGB and half AGB stars, tracing the primordial composition of the cluster. The chemical $s$-element abundances of probable AGB belonging to the $s$-poor group of M 22 could not reflect "in situ" phenomena, as the third dredge-up that would lead to enhanced $s$-process abundances in the atmosphere (and additionally is expected for much more massive AGB stars), but must reflect the primordial composition of the $s$-poor group. Moreover, we expect only a $\sim 10 \%$ contamination of giant population by AGB stars (Lloyd Evans 1975), hence it is unlikely that the $50 \%$ of stars in the $s$-poor sample of M09 are real AGB. From this discussion we conclude that the presence of few AGB stars does not influence our results, so for the present study we consider all the sample as composed by RGB, with a small con- 
tamination by AGB stars, without identifying individual candidate AGB stars.

To illuminate the relation between the $m_{1}$ values of $s$-rich and $s$-poor stars and their average $s$-element contents, we used the following procedure. First,we drew a ridge line for the blue RGB, by putting a spline through the median $m_{1}$ found in successive short intervals of $I$ magnitude, and obtained the dashed-dotted line shown in the upper-right panel of Fig. 19. We then calculated for each star its $m_{1}$ residuals from the ridge line (called here $\Delta m_{1}$ ). We plotted the $I$ magnitude versus $\Delta m_{1}$ as illustrated in the lower-left panel of Fig. 19. Finally, in the lower-right panel we show the average $s$-element abundance as a function of $\Delta m_{1}$. Clearly, the mean $\langle[s / \mathrm{Fe}]\rangle$ increases with increasing $\Delta m_{1}$.

\section{Summary and discussion}

We have presented a high resolution spectroscopic analysis of 35 RGB stars in the GC M22 from an heterogeneous sample of data, homogeneously analyzed. We have confirmed and extended the results of M09 that M 22 hosts two groups of stars whose mean $[\mathrm{Fe} / \mathrm{H}]$ metallicities differ by 0.15 dex (see also in DC09). These two groups turn out to have different chemical properties. First, they show a different $s / r$ abundance ratio. The two groups appear to be homogeneous in the $r$-process element $\mathrm{Eu}$, that is $\Delta_{\text {poor }}^{\text {rich }}[\mathrm{Eu} / \mathrm{Fe}] \sim 0$. However, the $s / r$ distribution, clearly traced by the $[\mathrm{La} / \mathrm{Eu}]$ abundance ratio, appears to be bimodal, with the $s$-rich stars having $\mathrm{La} / \mathrm{Eu}$ ratios about 2.5 times larger than in the $s$-poor ones, or $\Delta_{\text {poor }}^{\text {rich }}[\mathrm{La} / \mathrm{Eu}] \sim 0.4$. A bimodal split between the $s$-rich and $s$-poor of about the same amount with respect to $\mathrm{Eu}$ is observed also in the other $s$-process dominated species $(\mathrm{Ba}, \mathrm{Y}, \mathrm{Nd}, \mathrm{Zr}$ ). This demonstrates that the stars with higher metallicity are also more enriched in material processed through $s$ processes.

Since the most obvious M22 abundance anomaly is the spread in $s$-process abundances, it is good to re-emphasize that $[\mathrm{Eu} / \mathrm{Fe}]$ remains constant, within observational errors, independent of the Fe abundance. It is generally believed that massive stars are responsible for the Fe abundance and $\alpha$ elements. However, only a subset of these same stars appear to be responsible also for the production of $r$-process material. This can be seen in large-sample abundance surveys of metal-poor stars, which show relatively small star-to-star scatter in $[\alpha / \mathrm{Fe}]$ ratios but an enormous range in $[\mathrm{Eu} / \mathrm{Fe}]$ ratios (see the summary of many studies in Fig. 14 of Sneden et al. 2008). The constancy of $[\mathrm{Eu} / \mathrm{Fe}]$ points $\mathrm{M} 22$ to a common ratio of $r$-process-donating massive stars to $\mathrm{Fe}$ and $\alpha$-process stars in the ab initio IMF, irrespective of the Fe-metallicity of the two groups. This constancy is not easily achieved, and may require fine tuning of the evolutionary scenarios for M 22.

Evidence for a small increase in $[\mathrm{Cu} / \mathrm{Fe}]$ with increasing metallicity has been detected for our sample of stars: $s$-rich stars have higher $[\mathrm{Cu} / \mathrm{Fe}]$, while their $[\mathrm{Zn} / \mathrm{Fe}]$ values are essentially the same at all metallicities. This hints at an $s$-process contribution from the weak component in the production of $\mathrm{Cu}$, but the relatively large observational errors associated with $[\mathrm{Cu} / \mathrm{Fe}]$ suggests caution in this interpretation.

The relative abundances of the $\alpha$ elements ( $\mathrm{Si}$ and $\mathrm{Ti}$ ) with respect to $\mathrm{Fe}$ are constant, within observational errors, with metallicity. The curious trend of larger $[\mathrm{Ca} / \mathrm{Fe}]$ ratios by $\Delta_{\text {poor }}^{\text {rich }}[\mathrm{Ca} / \mathrm{Fe}] \sim 0.1$ in the higher metallicity M 22 stars found in M09, is confirmed here. Previous work, including Norris \& Freeman (1983) and Lehnert et al. (1991), saw a range in [Ca/H] of about $0.3-0.4$ dex. Our observed range in $[\mathrm{Ca} / \mathrm{H}]$ is larger than the one in $[\mathrm{Fe} / \mathrm{H}]$, and is comparable to what was reported in Norris \& Freeman (1983) and Lehnert et al. (1991). This increase in $[\mathrm{Ca} / \mathrm{Fe}]$ with $[\mathrm{Fe} / \mathrm{H}]$ is comparable to what is seen in $\omega$ Cen over a similar $[\mathrm{Fe} / \mathrm{H}]$ range (see two upper left panels of Fig. 12 of Johnson \& Pilachowski 2010). The difference in the mean $[\mathrm{Ca} / \mathrm{Fe}]$ in $\omega$ Cen between the metal-poor and the metalintermediate groups from Johnson \& Pilachowski is 0.08 dex (with a small mean error due to the large sample), which is very similar to the 0.1 dex difference we see in M 22 .

Among the light $p$-capture elements, M 22 stars show the same sort of $\mathrm{Na}-\mathrm{O}$ anticorrelation and $\mathrm{Na}-\mathrm{Al}$ correlations that have been extensively cataloged in mono-metallic clusters (e.g. Carretta et al. 2009b). This indicates that the $\mathrm{Ne} \rightarrow \mathrm{Na}$ and the $\mathrm{Mg} \rightarrow \mathrm{Al}$ conversions have been active in M22. The Na-O anticorrelation is present in each $s$-group, with $s$-rich and $s$-poor stars spanning a similar range in $[\mathrm{O} / \mathrm{Fe}]$, but a different range in $[\mathrm{Na} / \mathrm{Fe}]$, i.e. the average $[\mathrm{Na} / \mathrm{Fe}]$ at a given $[\mathrm{O} / \mathrm{Fe}]$ is higher in the $s$-rich stars than the $s$-poor by about 0.2 dex. Carbon and nitrogen reveal the typical anticorrelation expected from extensive $\mathrm{CN}$-cycle processing, but this becomes apparent only when we separate stars in the two $s$-groups. On average, the $s$-rich stars have higher $\mathrm{C}, \mathrm{N}$, and $\mathrm{Na}$ abundances, while they have similar $\mathrm{O}$ and $\mathrm{Mg}$. Hence, the average overall $\mathrm{CNO}$ abundance between the two $s$-groups differs by at least a factor of two.

The complexity of the chemical properties of M 22 also reflects on the CMD. A double RGB is visible when using the $m_{1}$ Strömgren index, sensitive to the $\mathrm{CN}$ bands and to metallicity (Richter et al. 1999): the lower [Fe/H] $s$-poor stars populate a sequence on the blue side, while the higher $[\mathrm{Fe} / \mathrm{H}] s$-rich ones obviously occupy a redder branch. A bimodal distribution is visible also on the SGB (Piotto 2009, M09), likely associated with the double RGB. We thus expect (but cannot prove with our data) that M22 SGB stars have the same bimodality in $s$-process elements and $\mathrm{Fe}$ exhibited by the giants studied in this work. If true, the overall $\mathrm{CNO}$ differences will play the dominant role in producing the SGB split, since M09 demonstrated that simple Fe-peak metallicity variations are not sufficient to generate enough of the observed photometric breadth in this region of the colour-magnitude diagram. Cassisi et al. (2008) and Ventura et al. (2009) have suggested that a bulk CNO abundance difference can account for a similar SGB split in NGC1851 (Milone et al. 2008). However, spectroscopic results for this cluster are contradictory, as evidence for $\mathrm{CNO}$ variations have been found by Yong et al. (2009), but not by Villanova et al. (2010).

Our work highlights the peculiarity of M22 among GCs. We consider "normal" GCs to be those that are: (i) essentially mono-metallic, i.e., all their stars appear to have the same $[\mathrm{Fe} / \mathrm{H}]$; (ii) chemically homogeneous in the heavy elements; but (iii) chemically inhomogeneous only in the light element abundances, as revealed by variations in the $\mathrm{CH}, \mathrm{CN}, \mathrm{NH}$ bands, and in the $\mathrm{O}, \mathrm{Na}, \mathrm{Al}$, and $\mathrm{Mg}$ abundances. Nearly all GCs have these characteristics. However, in M22, in addition to the O-Na anti-correlation there is a spread in the heavier elements, a characteristic that is seen only in a small number of other systems. Such systems include $\omega$ Cen, where the range in heavy elements is large and well established, the Galactic Bulge cluster Ter 5 (Ferraro et al. 2009), M 54, the central star cluster of the Sagittarius dwarf (Sarajedini \& Layden 1995; Bellazzini et al. 2008; Carretta et al. 2010a), the outer halo cluster NGC 2419 (Cohen et al. 2010), and perhaps NGC 1851 (Carretta et al. 2010b). The presence of heavy element abundance ranges in these systems necessarily means their nucleosynthetic history must be more complicated than for "normal" GCs, though whether it is an extension or a different process re- 
mains unclear. Current scenarios for explaining the abundance anomalies in "normal" GCs argue that polluters from a first stellar generation release into the intra-cluster medium large amounts of material from which a second generation could form. The candidate polluters are those expected to undergo the chemical processes responsible for the observed enrichment in $\mathrm{Na} / \mathrm{N} / \mathrm{Al}$ and depletions in $\mathrm{O}$ (and in some cases in $\mathrm{Mg}$ ). Candidate first-generation element donors include intermediate mass asymptotic giant branch (AGB) stars (D’Antona \& Caloi 2004), fast rotating massive stars (Decressin et al. 2007), and/or massive binaries (de Mink et al. 2009). Note also that Marcolini et al. (2009) have proposed an alternative scenario in which the first generation is the one with enhanced $\mathrm{Na}$ and depleted $\mathrm{O}$, at odds with what is proposed in most models.

It is difficult to fit M22's chemical properties into any of these cluster enrichment history proposals; its history has been more complicated than normal GCs. The pattern of differences in $[\mathrm{Fe} / \mathrm{H}]$, in the $s$-process dominated elements, and in $[\mathrm{Ca} / \mathrm{Fe}]$ in M 22 resembles the case of $\omega$ Cen, albeit in M 22 the range in $[\mathrm{Fe} / \mathrm{H}]$ is more than a factor of 20 lower. However, the M 22 $s$-process abundances apparently differ from those in $\omega$ Cen. In the latter, the $s$-process abundance ratios rise seemingly monotonically with $[\mathrm{Fe} / \mathrm{H}]$ before reaching a plateau at constant [s-process/Fe] (Norris \& Da Costa 1995a; Smith et al. 2000; Marino et al. 2011; Stanford et al. 2010; Johnson \& Pilachowski 2010). However, in M 22, while the range in [s-process/Fe] is comparable to that in $\omega$ Cen, our results do not suggest a monotonic increase of $[\mathrm{La} / \mathrm{Fe}]$ with $[\mathrm{Fe} / \mathrm{H}]$, rather there appears to be an overlap, possibly due to observational errors, in $[\mathrm{Fe} / \mathrm{H}]$ values between the $s$-poor and $s$-rich groups, with the location of the metal-rich, s-poor star II-31 could being particularly striking. These differences may suggest different nucleosynthetic histories for the two clusters, and could contrast with that proposed by Da Costa \& Marino (2010) who concluded that the $s$ enrichment processes in $\omega$ Cen and M 22 were similar, at least in the $[\mathrm{Fe} / \mathrm{H}]$ range common to both clusters. Indeed, aside from the spread in $[\mathrm{Fe} / \mathrm{H}]$ present in the two $s$-process groups, and their difference in $[\mathrm{Ca} / \mathrm{Fe}]$ values, it appears, considering the [s-process $/ \mathrm{Fe}]$ abundance ratios and those for other heavy elements in each group as constant, that two groups each behave separately in a similar way to normal mono-metallic GCs.

Interpretation of the M 22 abundance pattern would be much easier without the need to account for the $\mathrm{Ca}, \mathrm{Fe}$ and $s$-process variations. The presence of a group of stars with higher $[\mathrm{Fe} / \mathrm{H}]$ that also have little change in $\mathrm{Eu} / \mathrm{Fe}$ and most $\alpha / \mathrm{Fe}$ ratios could simply argue that multiple episodes of core-collapse supernova (SNII) played a role in the evolution of M 22. The larger [s-process/Fe] abundances in the higher metallicity M22 stars is a significant complication. In current self-enrichment models, the only ways to account for these observations is to consider an unique source for $\mathrm{Fe}$ and $s$-process elements, or a fine tuning in the times of accumulation of the material from which successive generations form or not evolving as an isolated system so that external gas flows can contribute to the enrichment processes.

In the Sun, the $s$-process contribution is mainly due to two components: $(i)$ the "main" component attributed to low mass AGB stars ( 2-4 $M_{\odot}$; Busso et al. 1999), and (ii) the "weak" component attributed to massive stars (Raiteri et al. 1993, and references therein). If the $s$-process enrichment in the $s$-rich stars in M 22 is due to the main component, this would imply a relatively large difference in age among the $s$-rich and $s$-poor stars, since the low mass AGB stars evolve in times even of the order of Gyrs. Low mass AGB stars are also expected to increase the total CNO abundances, which would be consistent with our re- sults. A major $s$-process contribution from the weak component would be consistent with a much faster evolution of the cluster, and with a smaller age difference between the $s$-groups. Note that the weak component mainly produces the lighter nuclei in the $s$-chain, like ${ }^{58} \mathrm{Fe},{ }^{63} \mathrm{Cu}$, and ${ }^{65} \mathrm{Cu}$. Since, as suggested by Sneden et al. (1991), much of the $\mathrm{Cu}$ in metal-poor stars can be produced in the weak component of the $s$-processes, a possible $\mathrm{Cu}$ increase with $\mathrm{Fe}$ qualitatively supports the idea that massive stars contribute to the pollution of the intra-cluster medium in M 22. The advantage of this scenario is that the same stars can be the sources for increases in the metallicity and $s$-process elements. However, if this scenario is correct it would imply that massive stars also produce enough of the heavier $s$-process nuclei to quantitatively account for the observed $\mathrm{Ba}, \mathrm{La}$, and $\mathrm{Nd}$ abundances in the $s$-rich group of M 22 stars. Simply following this scenario, after the evolution of massive stars belonging to the metal/s-process-poor group (possibly from both Na-poor/O-rich and Na-rich/O-poor populations), a second generation slightly enhanced in $\mathrm{Fe}$, and enriched in $s$-process elements formed from the material created by these massive stars that end as SNII. Then, the Na-poor/O-rich stars from the $s$-rich stars form their own $\mathrm{Na}-\mathrm{O}$ anticorrelation (and Al-Na correlations), similarly to normal GCs.

Instructive at this point, is again the comparison with $\omega$ Cen, and its constancy in $[\mathrm{Cu} / \mathrm{Fe}]$. In this extreme cluster the enormous elemental variations in $n$-capture have been interpreted as due to the contributions from low-mass AGB stars via $s$-process nucleosynthesis (e.g., Norris \& Da Costa 1995a), thus the observed constant values of $[\mathrm{Cu} / \mathrm{Fe}]$ found by Cunha et al. (2002) do not fit a picture in which $\mathrm{Cu}$ is produced in AGB stars. This could mean that we shouldn't be looking to the mechanism that produces the $s$-process abundance difference between the two M 22 groups to explain the $[\mathrm{Cu} / \mathrm{Fe}]$. In turn, this could suggest that in M22 we may well have evidence that weak-s in massive stars is contributing to the nucleosynthesis, and possibly is not in $\omega$ Cen. As previously said, we express caution with our results on $[\mathrm{Cu} / \mathrm{Fe}]$ abundances as the associated observational errors are relatively large. However, the contribution from the weak- $s$ component in M 22 could be supported also by the lower [heavy-s/light- $s]$ ratio, traced by the $[\mathrm{La} / \mathrm{Y}]$ abundances, in the $s$-rich stars that have also higher $[\mathrm{Cu} / \mathrm{Fe}]$. Indeed, the weak- $s$ component produces more light- $s$, than heavier $s$-process elements.

As an alternative scenario, we could suppose the following sequence of events: 1) SNII in the cluster explode and expel material far from the cluster center at high velocity; 2) intermediate AGB stars pollute the medium of material enriched in $\mathrm{Na} / \mathrm{N} / \mathrm{Al}$ and depleted in $\mathrm{O}$, this material goes into the cluster central region via cooling flow (as predicted by D'Ercole et al. 2008), hence a second generation of stars (Na-rich, O-poor) formed; 3 ) at the end, low mass AGB stars evolve and expelled material enriched in $s$-process-elements. A second cooling flow involves the material ejected from low mass AGB and the one expelled by the first SNII. The latter should need a longer time to be reattracted towards the center of the cluster because it was ejected at higher velocity. The material from low mass AGB and SNII mix together in the center of the cluster, and another star formation event occurs forming stars enriched in $s$ elements and $\mathrm{Fe}$ at the same time.

These evolutionary scenarios require special circumstances to occur for M 22 but not for the vast majority of GCs. Another qualitative attempt to explain M 22's abundance set is to suppose that the present M 22 is composed of the merger of two originally clusters. Of course, in this hypothesis we are assuming that any 
spread is present in the $[\mathrm{Fe} / \mathrm{H}]$ abundances in each $s$-process group, and hence the two $s$-groups being mono-metallic. This is attractive because it does not require now-departed members of the more metal-poor group to have been responsible for the creation of the more metal-rich group. This idea would probably fail immediately if no normal GCs could be found with the chemical mixes of M 22's two stellar groups. However the well-studied M 5 (Ivans et al. 2001; Ramírez \& Cohen 2003) and M 4 (Ivans et al. 1999; Marino et al. 2008) have relative abundance mixes that resemble those of the lower and higher metallicity M 22 groups, respectively. The mean literature values for $\mathrm{Ba}$ and $\mathrm{La}$ in M 5 range from -0.08 to 0.18 , and 0.02 to 0.18 , respectively. On the other hand, M 4 has substantially higher values of these abundance ratios: $[\mathrm{La} / \mathrm{Fe}]=0.45,[\mathrm{Ba} / \mathrm{Fe}]=0.60$ (Ivans et al. 1999); $[\mathrm{Ba} / \mathrm{Fe}]=0.41$ (Marino et al. 2008); these are unusually high for GCs. Additionally, the $[\mathrm{Fe} / \mathrm{H}]$ metallicity difference between M 5 and M 4 is about 0.1 dex, similar to the mean difference in the two M 22 groups. However, that spread is not a requirement of the cluster merger scenario. Finally, the mean $\mathrm{Na}$ and Al abundances are slightly higher in M4 than in M 5. A significant uncertainty for the cluster merging idea might be the unknown probability of such a merger to have happened early in our Galaxy's history. The merger probability of two GCs in the field halo is likely to be very small given the volume, but that may not true in a dwarf galaxy/merger object - there the clusters exist in a much smaller volume, the relative velocities are lower and maybe dynamical friction can bring two clusters together in the center, with the dwarf galaxy subsequently disrupted (see Bekki 2010, and references therein).

Another GC has been recently interpreted in cluster merging hypothesis: NGC 1851 (Carretta et al. 2010b). Like M 22, there is a bimodality in $s$-process elements (Yong \& Grundahl 2008), a possible spread in $[\mathrm{Fe} / \mathrm{H}]$ metallicity and $[\mathrm{Ca} / \mathrm{H}]$, a segregation of stars in color-magnitude diagram quantities that correlates with abundance variations (Milone et al. 2008; Han et al. 2009).

Our findings show that M 22 represents an important piece in the understanding of GC evolution. It shares similarities both with normal mono-metallic GCs and with the most extreme case of $\omega$ Cen, and thus may be a bridge to better understanding what has made $\omega$ Cen so unique. A full understanding of the M 22 chemical evolution should be important in shedding light on the multiple stellar population phenomenon in GCs.

Acknowledgements. We are grateful for the observing time allocated to this program by the directors and telescope allocation committees of several observatories. G.W. and C.S. thank the Padua Astronomical Observatory for its hospitality while parts of this paper were being completed. We thank Melike Afşar, Evan Kirby, and Ian Roederer for comments and suggestions on the manuscript, B. Plez \& V. Smith for providing their linelists for molecular bands, K. Lind and M. Bergemann for useful discussions on NLTE abundances, and the referee for his/her suggestions that have improved the manuscript. This publication makes use of data products from: (a) the Two Micron All Sky Survey, which is a joint project of the University of Massachusetts and the Infrared Processing and Analysis Center/California Institute of Technology, funded by the National Aeronautics and Space Administration and the National Science Foundation; and (b) the Canadian Astronomy Data Centre, operated by the National Research Council of Canada with the support of the Canadian Space Agency. This research has made use of the SIMBAD database, operated at CDS, Strasbourg, France. We gratefully acknowledge the sources of financial support for this study: to A.F.M., A.P.M., and G.P. for PRIN2007 (grant prot. No. 20075TP5K9); to M.Z. for Fondecyt Regular 1085278 and the Mideplan Milky Way Millennium Nucleus P07-021-F; to C.S. for several grants from the US National Science Foundation, currently AST 09-08978; and to J.E.N. for Australian Research Council grant DP0984924 for studies of the populations of Galactic stellar systems.

\section{References}

Anderson, J., Piotto, G., King, I. R., Bedin, L. R., \& Guhathakurta, P. 2009, ApJ, 697, L58

Arp, H. C., \& Melbourne, W. G. 1959, AJ, 64, 28

Alonso, A., Arribas, S., \& Martinez-Roger, C. 1999, A\&A, 140, 261

Alonso, A., Arribas, S., \& Martínez-Roger, C. 2001, A\&A, 376, 1039

Bedin, L. R., Piotto, G., Anderson, J., et al. 2004, ApJ, 605, L125

Bekki, K. 2010, MNRAS, 401, 2753

Bellazzini, M., Ibata, R. A., Chapman, S. C., et al. 2008, AJ, 136, 1147

Bergemann, M. 2011, MNRAS, 413, 2184

Brown, J. A., Wallerstein, G., \& Oke, J. B. 1990, AJ, 100, 1561

Busso, M., Gallino, R., \& Wasserburg, G. J. 1999, ARA\&A, 37, 239

Cardelli, J. A., Clayton, G. C., \& Mathis, J. S. 1989, ApJ, 345, 245

Carretta, E., Bragaglia, A., Gratton, R., D’Orazi, V., \& Lucatello, S. 2009a, A\&A, 508, 695

Carretta, E., Bragaglia, A., Gratton, R. G., et al. 2009b, A\&A, 505, 117

Carretta, E., Bragaglia, A., Gratton, R. G., et al. 2010a, ApJ, 714, L7

Carretta, E., Gratton, R. G., Lucatello, S., et al. 2010b, ApJ, 722, L1

Cassisi, S., Salaris, M., Pietrinferni, A., et al. 2008, ApJ, 672, L115

Castelli, F., \& Kurucz, R. L. 2004 [arXiv: astro-ph/0405087]

Cohen, J. G. 1981, ApJ, 247, 869

Cohen, J. G., Kirby, E. N., Simon, J. D., \& Geha, M. 2010, ApJ, 725, 288

Couch, R. G., Schmiedekamp, A. B., \& Arnett, W. D. 1974, ApJ, 190, 95

Cudworth, K. M. 1986, AJ, 92, 348

Cunha, K., Smith, V. V., Suntzeff, N. B., et al. 2002, AJ, 124, 379

D’Antona, F., \& Caloi, V. 2004, ApJ, 611, 871

D'Antona, F., \& Ventura, P. 2007, MNRAS, 379, 1431

Da Costa, G. S., Held, E. V., Saviane, I., \& Gullieuszik, M. 2009, ApJ, 705, 1481

Da Costa, G. S., \& Marino, A. F. 2011, PASA, 28, 28

Decressin, T., Meynet, G., Charbonnel, C., Prantzos, N., \& Ekström, S. 2007, A\&A, 464, 1029

de Mink, S. E., Pols, O. R., Langer, N., \& Izzard, R. G. 2009, A\&A, 507, L1

D'Ercole, A., Vesperini, E., D'Antona, F., McMillan, S. L. W., \& Recchi, S. 2008, MNRAS, 391, 825

Dekker, H., D’Odorico, S., Kaufer, A., Delabre, B., \& Kotzlowski, H. 2000, Proc. SPIE, 4008, 534

Diego, F., Charalambous, A., Fish, A. C., \& Walker, D. D. 1990, Proc. SPIE, 1235,562

Ferraro, F. R., Dalessandro, E., Mucciarelli, A., et al. 2009, Nature, 462, 483

Frogel, J. A., Persson, S. E., \& Cohen, J. G. 1983, ApJS, 53, 713

Goodrich, R. W., \& Veilleux, S. 1988, PASP, 100, 1572

Gratton, R. G. 1982, A\&A, 115, 171

Han, S.-I., Lee, Y.-W., Joo, S.-J., et al. 2009, ApJ, 707, L190

Harris, W. E. 1996, AJ, 112, 1487

Helfer, H. L., Wallerstein, G., \& Greenstein, J. L. 1959, ApJ, 129, 700

Hesser, J. E., Hartwick, F. D. A., \& McClure, R. D. 1977, ApJS, 33, 471

Hill, V., et al. 2002, A\&A, 387, 560

Ivans, I. I., Sneden, C., Kraft, R. P., et al. 1999, AJ, 118, 1273

Ivans, I. I., Kraft, R. P., Sneden, C., et al. 2001, AJ, 122, 1438

Johnson, C. I., \& Pilachowski, C. A. 2010, ApJ, 722, 1373

Kayser, A., Hilker, M., Grebel, E. K., \& Willemsen, P. G. 2008, A\&A, 486, 437

Kraft, R. P., \& Ivans, I. I. 2003, PASP, 115, 143

Kurucz, R. L. 1992, in The Stellar Populations of Galaxies, ed. B. Barbuy, \& A. Renzini (Dordrecht: Reidel), IAU Symp., 149, 225

Kurucz, R. L. 2009, in Recent Directions in Astrophysical Quantitative Spectroscopy and Radiation Hydrodynamics, Am. Inst. Phys. Conf. Ser., 1171,43

Kučinskas, A., Hauschildt, P. H., Brott, I., et al. 2006, A\&A, 452, 1021

Lawler, J. E., Bonvallet, G., \& Sneden, C. 2001a, ApJ, 556, 452

Lawler, J. E., Wickliffe, M. E., den Hartog, E. A., \& Sneden, C. 2001b, ApJ, 563, 1075

Laws, C., \& Gonzalez, G. 2003, ApJ, 595, 1148

Lee, J.-W., Kang, Y.-W., Lee, J., \& Lee, Y.-W. 2009, Nature, 462, 480

Lehnert, M. D., Bell, R. A., \& Cohen, J. G. 1991, ApJ, 367, 514

Lind, K., Charbonnel, C., Decressin, T., et al. 2011a, A\&A, 527, A148

Lind, K., Asplund, M., Barklem, P. S., \& Belyaev, A. K. 2011b, A\&A, 528, A103

Lloyd Evans, T. 1975, MNRAS, 171, 647

Marcolini, A., Gibson, B. K., Karakas, A. I., \& Sánchez-Blázquez, P. 2009, MNRAS, 395, 719

Marino, A. F., Villanova, S., Piotto, G., et al. 2008, A\&A, 490, 625

Marino, A. F., Milone, A. P., Piotto, G., et al. 2009, A\&A, 505, 109

Marino, A. F., Milone, A. P., Piotto, G., et al. 2011, ApJ, 731, 64

Milone, A. P., Bedin, L. R., Piotto, G., et al. 2008, ApJ, 673, 241

Milone, A. P., Bedin, L. R., Piotto, G., \& Anderson, J. 2009, A\&A, 497, 755

Milone, A. P., Piotto, G., King, I. R., et al. 2010, ApJ, 709, 1183

Milone, A. P., et al. 2011, A\&A, submitted 
A. F. Marino et al.: The two metallicity groups in M 22

Mishenina, T. V., Kovtyukh, V. V., Soubiran, C., Travaglio, C., \& Busso, M. 2002, A\&A, 396, 189

Norris, J., \& Freeman, K. C. 1983, ApJ, 266, 130

Norris, J. E., \& Da Costa, G. S. 1995a, ApJ, 447, 680

Norris, J. E., Da Costa, G. S., \& Tingay, S. J. 1995b, ApJS, 99, 637

Peterson, R. C., \& Cudworth, K. M. 1994, ApJ, 420, 612

Pilachowski, C., Leep, E. M., Wallerstein, G., \& Peterson, R. C. 1982, ApJ, 263, 187

Piotto, G., Bedin, L. R., Anderson, J., et al. 2007, ApJ, 661, L53

Piotto, G. 2009, IAU Symp., 258, 233

Raiteri, C. M., Busso, M., Picchio, G., Gallino, R., \& Pulone, L. 1991, ApJ, 367, 228

Raiteri, C. M., Gallino, R., Busso, M., Neuberger, D., \& Kaeppeler, F. 1993, ApJ, 419, 207

Ramírez, S. V., \& Cohen, J. G. 2003, AJ, 125, 224

Richter, P., Hilker, M., \& Richtler, T. 1999, A\&A, 350, 476

Sarajedini, A., \& Layden, A. C. 1995, AJ, 109, 1086

Sarajedini, A., Bedin, L. R., Chaboyer, B., et al. 2007, AJ, 133, 1658

Simmerer, J., Sneden, C., Ivans, I. I., et al. 2003, AJ, 125, 2018
Simmerer, J., Sneden, C., Cowan, J. J., et al. 2004, ApJ, 617, 1091 Skrutskie, M. F., Cutri, R. M., Stiening, R., et al. 2006, AJ, 131, 1163 Smith, V. V., \& Suntzeff, N. B. 1989, AJ, 97, 1699

Smith, V. V., Suntzeff, N. B., Cunha, K., et al. 2000, AJ, 119, 1239 Sneden, C. A. 1973, Ph.D. Thesis

Sneden, C., Gratton, R. G., \& Crocker, D. A. 1991, A\&A, 246, 354

Sneden, C., Cowan, J. J., \& Gallino, R. 2008, ARA\&A, 46, 241

Tull, R. G., MacQueen, P. J., Sneden, C., \& Lambert, D. L. 1995, PASP, 107, 251

Stanford, L. M., Da Costa, G. S., \& Norris, J. E. 2010, ApJ, 714, 1001

Valenti, J. A., Butler, R. P., \& Marcy, G. W. 1995, PASP, 107, 966

Ventura, P., D'Antona, F., Mazzitelli, I., \& Gratton, R. 2001, ApJ, 550, L65

Ventura, P., Caloi, V., D'Antona, F., et al. 2009, MNRAS, 399, 934

Vogt, S. S. 1987, PASP, 99, 1214

Villanova, S., Geisler, D., \& Piotto, G. 2010, ApJ, 722, L18

Yong, D., \& Grundahl, F. 2008, ApJ, 672, L29

Yong, D., Grundahl, F., D’Antona, F., et al. 2009, ApJ, 695, L62

Wang, S.-I., Hildebrand, R. H., Hobbs, L. M., et al. 2003, Proc. SPIE, 4841, 1145

Pages 20 to 24 are available in the electronic edition of the journal at http://www. aanda. org 
A\&A 532, A8 (2011)

Table 3. Atmospheric parameters.

\begin{tabular}{|c|c|c|c|c|c|}
\hline Star & Source & $\begin{array}{l}T_{\text {eff }} \\
{[\mathrm{K}]}\end{array}$ & $\log g$ & $\begin{array}{c}\xi_{\mathrm{t}} \\
{\left[\mathrm{km} \mathrm{s}^{-1}\right]}\end{array}$ & {$[\mathrm{Fe} / \mathrm{H}]$} \\
\hline \multirow[t]{4}{*}{ I-12 } & MCD & 4260 & 0.45 & 1.55 & -1.90 \\
\hline & AAT & 4300 & 0.70 & 1.70 & -1.86 \\
\hline & UVES & 4300 & 0.75 & 1.55 & -1.85 \\
\hline & AVG. & 4285 & 0.65 & 1.60 & -1.87 \\
\hline \multirow{3}{*}{$\mathrm{I}-27$} & APO & 4420 & 1.40 & 1.60 & -1.82 \\
\hline & UVES & 4490 & 1.46 & 1.65 & -1.63 \\
\hline & AVG. & 4455 & 1.45 & 1.60 & -1.73 \\
\hline I-36 & LICK & 4400 & 0.80 & 1.70 & -1.89 \\
\hline I-37 & LICK & 4370 & 0.90 & 1.55 & -1.73 \\
\hline I-53 & UVES & 4500 & 1.35 & 1.55 & -1.74 \\
\hline \multirow[t]{3}{*}{ I-57 } & APO & 4300 & 1.20 & 1.70 & -1.65 \\
\hline & AAT & 4250 & 0.90 & 1.65 & -1.62 \\
\hline & AVG. & 4275 & 1.05 & 1.65 & -1.64 \\
\hline I-80 & UVES & 4460 & 1.15 & 1.55 & -1.70 \\
\hline I-85 & UVES & 4600 & 1.00 & 1.45 & -1.81 \\
\hline \multirow[t]{3}{*}{ I-86 } & AAT & 4420 & 1.10 & 1.20 & -1.80 \\
\hline & UVES & 4500 & 1.30 & 1.50 & -1.84 \\
\hline & AVG. & 4460 & 1.20 & 1.35 & -1.82 \\
\hline I-92 & LICK & 4240 & 0.75 & 1.55 & -1.75 \\
\hline II-1 & LICK & 4300 & 0.75 & 1.50 & -1.66 \\
\hline II-31 & LICK & 4380 & 1.20 & 1.65 & -1.65 \\
\hline II-96 & MCD & 4400 & 1.00 & 2.10 & -1.82 \\
\hline II-104 & UVES & 4460 & 1.15 & 1.45 & -1.76 \\
\hline \multirow[t]{4}{*}{ III-3 } & MCD & 4000 & 0.30 & 2.25 & -1.72 \\
\hline & APO & 4010 & 0.40 & 2.25 & -1.78 \\
\hline & UVES & 3990 & 0.20 & 2.10 & -1.66 \\
\hline & AVG. & 4000 & 0.30 & 2.20 & -1.72 \\
\hline \multirow[t]{3}{*}{ III-12 } & MCD & 4150 & 0.70 & 1.95 & -1.69 \\
\hline & AAT & 4220 & 1.25 & 2.00 & -1.61 \\
\hline & AVG. & 4185 & 1.00 & 1.95 & -1.65 \\
\hline \multirow[t]{3}{*}{ III-14 } & LICK & 4010 & 0.40 & 2.15 & -1.84 \\
\hline & AAT & 4050 & 0.30 & 2.15 & -1.80 \\
\hline & AVG. & 4030 & 0.35 & 2.15 & -1.82 \\
\hline III-15 & LICK & 4070 & 0.40 & 1.85 & -1.82 \\
\hline III-25 & UVES & 4700 & 1.35 & 1.75 & -1.92 \\
\hline III-33 & UVES & 4430 & 1.05 & 1.70 & -1.78 \\
\hline III-35 & UVES & 4500 & 1.25 & 1.35 & -1.83 \\
\hline III-47 & APO & 4600 & 1.20 & 2.00 & -1.82 \\
\hline III-50 & UVES & 4700 & 1.70 & 1.45 & -1.76 \\
\hline \multirow[t]{3}{*}{ III-52 } & LICK & 4050 & 0.50 & 1.70 & -1.63 \\
\hline & UVES & 4100 & 0.65 & 1.80 & -1.62 \\
\hline & AVG. & 4075 & 0.60 & 1.75 & -1.63 \\
\hline III-96 & AAT & 4480 & 1.30 & 1.65 & -1.86 \\
\hline \multirow[t]{3}{*}{ IV-20 } & AAT & 4320 & 1.05 & 1.70 & -1.65 \\
\hline & UVES & 4260 & 0.90 & 1.60 & -1.63 \\
\hline & AVG. & 4290 & 1.00 & 1.65 & -1.64 \\
\hline IV-59 & UVES & 4400 & 1.00 & 1.70 & -1.77 \\
\hline IV-68 & UVES & 4750 & 1.65 & 1.20 & -1.75 \\
\hline IV-76 & AAT & 4730 & 1.50 & 2.30 & -1.63 \\
\hline IV-88 & AAT & 4400 & 1.20 & 1.70 & -1.62 \\
\hline IV-97 & UVES & 4000 & 0.05 & 2.00 & -1.94 \\
\hline \multirow[t]{4}{*}{ IV-102 } & LICK & 4020 & 0.25 & 2.15 & -1.96 \\
\hline & MCD & 4050 & 0.10 & 2.35 & -1.95 \\
\hline & AAT & 3990 & 0.20 & 2.15 & -2.01 \\
\hline & AVG. & 4020 & 0.20 & 2.20 & -1.97 \\
\hline $\mathrm{C}$ & $\mathrm{MCD}$ & 3960 & 0.30 & 2.25 & -1.69 \\
\hline C513 & APO & 4100 & 0.40 & 1.65 & -1.86 \\
\hline $\mathrm{V}-2$ & LICK & 4130 & 0.65 & 1.75 & -1.57 \\
\hline
\end{tabular}


A. F. Marino et al.: The two metallicity groups in M 22

Table 4. Sensitivity of abundance ratios on atmospheric parameters changes.

\begin{tabular}{|c|c|c|c|c|c|}
\hline$\overline{[\mathrm{X} / \mathrm{Fe}]^{a}}$ & 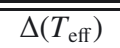 & $\overline{\overline{\Delta(\log g)}}$ & $\overline{\overline{\Delta([\mathrm{A} / \mathrm{H}])}}$ & 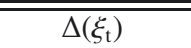 & $\overline{\text { Total }^{b}}$ \\
\hline \multicolumn{6}{|c|}{ AAT: IV-20 } \\
\hline & $\pm 60 \mathrm{~K}$ & \pm 0.15 & \pm 0.10 & $\pm 0.12 \mathrm{~km} \mathrm{~s}^{-1}$ & \\
\hline $\mathrm{O}$ & \pm 0.00 & \pm 0.05 & $\mp 0.06$ & \pm 0.00 & 0.08 \\
\hline $\mathrm{Na}$ & \pm 0.05 & $\mp 0.02$ & $\mp 0.10$ & $\mp 0.03$ & 0.12 \\
\hline $\mathrm{Mg}$ & $\mp 0.06$ & $\mp 0.01$ & \pm 0.00 & \pm 0.03 & 0.07 \\
\hline $\mathrm{Al}$ & $\mp 0.05$ & $\mp 0.01$ & \pm 0.00 & \pm 0.04 & 0.06 \\
\hline $\mathrm{Si}$ & $\mp 0.09$ & \pm 0.02 & \pm 0.02 & \pm 0.05 & 0.11 \\
\hline $\mathrm{Ca}$ & $\mp 0.03$ & $\mp 0.02$ & \pm 0.00 & \pm 0.00 & 0.04 \\
\hline $\mathrm{Ti}_{\mathrm{I}}$ & \pm 0.00 & $\mp 0.02$ & \pm 0.00 & \pm 0.03 & 0.04 \\
\hline $\mathrm{Fe}_{\mathrm{I}}$ & \pm 0.11 & \pm 0.00 & $\mp 0.01$ & $\mp 0.05$ & 0.12 \\
\hline $\mathrm{Fe}_{\mathrm{II}}$ & $\mp 0.05$ & \pm 0.07 & \pm 0.02 & $\mp 0.02$ & 0.09 \\
\hline $\mathrm{Cu}$ & \pm 0.10 & \pm 0.00 & $\mp 0.10$ & \pm 0.00 & 0.14 \\
\hline $\mathrm{Ba}$ & $\mp 0.07$ & \pm 0.04 & \pm 0.04 & $\mp 0.05$ & 0.10 \\
\hline $\mathrm{La}$ & \pm 0.02 & \pm 0.07 & $\mp 0.06$ & \pm 0.00 & 0.09 \\
\hline \multicolumn{6}{|c|}{ APO: I-57 } \\
\hline & $\pm 100 \mathrm{~K}$ & \pm 0.17 & \pm 0.10 & $\pm 0.13 \mathrm{~km} \mathrm{~s}^{-1}$ & \\
\hline $\mathrm{O}$ & \pm 0.03 & \pm 0.07 & $\mp 0.05$ & \pm 0.00 & 0.09 \\
\hline $\mathrm{Na}$ & \pm 0.12 & $\mp 0.00$ & $\mp 0.10$ & \pm 0.00 & 0.16 \\
\hline $\mathrm{Mg}$ & $\mp 0.05$ & $\mp 0.03$ & $\mp 0.03$ & \pm 0.02 & 0.07 \\
\hline $\mathrm{Al}$ & $\mp 0.05$ & $\mp 0.01$ & $\mp 0.01$ & \pm 0.04 & 0.07 \\
\hline $\mathrm{Si}$ & $\mp 0.13$ & \pm 0.00 & $\mp 0.01$ & \pm 0.03 & 0.13 \\
\hline $\mathrm{Ca}$ & \pm 0.00 & $\mp 0.04$ & $\mp 0.03$ & $\mp 0.02$ & 0.05 \\
\hline $\mathrm{Ti}_{\mathrm{I}}$ & \pm 0.09 & $\mp 0.03$ & $\mp 0.03$ & \pm 0.00 & 0.10 \\
\hline $\mathrm{Ti}_{\mathrm{II}}$ & $\mp 0.12$ & \pm 0.02 & \pm 0.00 & $\mp 0.04$ & 0.13 \\
\hline $\mathrm{Fe}_{\mathrm{I}}$ & \pm 0.13 & \pm 0.01 & \pm 0.01 & $\mp 0.04$ & 0.14 \\
\hline $\mathrm{Fe}_{\text {II }}$ & $\mp 0.08$ & \pm 0.07 & \pm 0.09 & $\mp 0.01$ & 0.14 \\
\hline $\mathrm{Cu}$ & \pm 0.06 & \pm 0.00 & $\mp 0.10$ & \pm 0.00 & 0.12 \\
\hline $\mathrm{Zn}$ & \pm 0.05 & \pm 0.02 & $\mp 0.10$ & $\mp 0.06$ & 0.13 \\
\hline $\mathrm{Y}$ & $\mp 0.11$ & \pm 0.03 & \pm 0.00 & \pm 0.01 & 0.11 \\
\hline $\mathrm{Zr}$ & \pm 0.00 & \pm 0.05 & $\mp 0.04$ & \pm 0.00 & 0.06 \\
\hline $\mathrm{Ba}$ & $\mp 0.10$ & \pm 0.04 & \pm 0.03 & $\mp 0.06$ & 0.13 \\
\hline $\mathrm{La}$ & \pm 0.05 & \pm 0.08 & $\mp 0.06$ & \pm 0.00 & 0.11 \\
\hline $\mathrm{Nd}$ & $\mp 0.10$ & \pm 0.04 & \pm 0.01 & \pm 0.02 & 0.11 \\
\hline $\mathrm{Eu}$ & \pm 0.00 & \pm 0.08 & $\mp 0.05$ & \pm 0.00 & 0.09 \\
\hline \multicolumn{6}{|c|}{ LICK: II-31 } \\
\hline & $\pm 70 \mathrm{~K}$ & \pm 0.18 & \pm 0.10 & $\pm 0.12 \mathrm{~km} \mathrm{~s}^{-1}$ & \\
\hline $\mathrm{O}$ & \pm 0.00 & \pm 0.08 & $\mp 0.05$ & \pm 0.00 & 0.09 \\
\hline $\mathrm{Na}$ & \pm 0.05 & $\mp 0.02$ & $\mp 0.10$ & \pm 0.02 & 0.12 \\
\hline $\mathrm{Mg}$ & $\mp 0.03$ & $\mp 0.01$ & \pm 0.02 & \pm 0.02 & 0.04 \\
\hline $\mathrm{Al}$ & $\mp 0.01$ & $\mp 0.01$ & \pm 0.01 & \pm 0.03 & 0.03 \\
\hline $\mathrm{Si}$ & $\mp 0.08$ & \pm 0.02 & \pm 0.01 & \pm 0.03 & 0.09 \\
\hline $\mathrm{Ca}$ & \pm 0.02 & $\mp 0.01$ & \pm 0.03 & $\mp 0.02$ & 0.04 \\
\hline $\mathrm{Ti}_{\mathrm{I}}$ & \pm 0.03 & $\mp 0.01$ & \pm 0.01 & $\mp 0.01$ & 0.03 \\
\hline $\mathrm{Ti}_{\mathrm{II}}$ & $\mp 0.10$ & \pm 0.06 & $\mp 0.01$ & $\mp 0.01$ & 0.12 \\
\hline $\mathrm{Fe}_{\mathrm{I}}$ & \pm 0.09 & \pm 0.00 & $\mp 0.01$ & $\mp 0.03$ & 0.10 \\
\hline $\mathrm{Fe}_{\mathrm{II}}$ & $\mp 0.06$ & \pm 0.08 & \pm 0.02 & $\mp 0.01$ & 0.10 \\
\hline $\mathrm{Cu}$ & \pm 0.04 & \pm 0.00 & $\mp 0.05$ & \pm 0.00 & 0.06 \\
\hline $\mathrm{Zn}$ & \pm 0.00 & \pm 0.02 & $\mp 0.05$ & $\mp 0.02$ & 0.06 \\
\hline $\mathrm{Y}$ & $\mp 0.09$ & \pm 0.06 & $\mp 0.03$ & $\mp 0.03$ & 0.12 \\
\hline $\mathrm{Zr}$ & \pm 0.00 & \pm 0.10 & $\mp 0.05$ & \pm 0.00 & 0.11 \\
\hline $\mathrm{Ba}$ & $\mp 0.07$ & \pm 0.05 & \pm 0.05 & $\mp 0.06$ & 0.12 \\
\hline $\mathrm{La}$ & \pm 0.00 & \pm 0.07 & $\mp 0.05$ & \pm 0.00 & 0.09 \\
\hline $\mathrm{Nd}$ & $\mp 0.08$ & \pm 0.06 & \pm 0.04 & \pm 0.02 & 0.11 \\
\hline $\mathrm{Eu}$ & \pm 0.00 & \pm 0.06 & $\mp 0.08$ & \pm 0.00 & 0.10 \\
\hline \multicolumn{6}{|c|}{ MCD: II-96 } \\
\hline & $\pm 50 \mathrm{~K}$ & \pm 0.10 & \pm 0.10 & $\pm 0.12 \mathrm{~km} \mathrm{~s}^{-1}$ & \\
\hline $\mathrm{O}$ & \pm 0.00 & \pm 0.05 & $\mp 0.04$ & \pm 0.00 & 0.06 \\
\hline $\mathrm{Na}$ & \pm 0.05 & $\mp 0.00$ & $\mp 0.10$ & \pm 0.00 & 0.11 \\
\hline $\mathrm{Mg}$ & \pm 0.02 & $\mp 0.02$ & \pm 0.00 & \pm 0.02 & 0.03 \\
\hline
\end{tabular}


A\&A 532, A8 (2011)

Table 4. continued.

\begin{tabular}{lccccc}
\hline \hline$[\mathrm{X} / \mathrm{Fe}]^{a}$ & $\Delta\left(T_{\text {eff }}\right)$ & $\Delta(\log g)$ & $\Delta([\mathrm{A} / \mathrm{H}])$ & $\Delta\left(\xi_{\mathrm{t}}\right)$ & Total $^{b}$ \\
& & & & & \\
\hline $\mathrm{Al}$ & $\mp 0.03$ & $\mp 0.01$ & \pm 0.00 & \pm 0.02 & 0.04 \\
$\mathrm{Si}$ & \pm 0.05 & \pm 0.00 & \pm 0.02 & \pm 0.02 & 0.06 \\
$\mathrm{Ca}$ & \pm 0.01 & $\mp 0.01$ & \pm 0.00 & \pm 0.02 & 0.02 \\
$\mathrm{Ti}_{\mathrm{I}}$ & $\mp 0.02$ & $\mp 0.02$ & \pm 0.01 & \pm 0.01 & 0.03 \\
$\mathrm{Ti}_{\mathrm{II}}$ & \pm 0.09 & \pm 0.04 & \pm 0.04 & \pm 0.00 & 0.11 \\
$\mathrm{Fe}_{\mathrm{I}}$ & \pm 0.07 & \pm 0.00 & $\mp 0.02$ & $\mp 0.03$ & 0.08 \\
$\mathrm{Fe}_{\mathrm{II}}$ & $\mp 0.04$ & \pm 0.08 & \pm 0.02 & $\mp 0.01$ & 0.09 \\
$\mathrm{Cu}$ & \pm 0.05 & \pm 0.00 & $\mp 0.05$ & $\mp 0.06$ & 0.09 \\
$\mathrm{Zn}$ & \pm 0.00 & \pm 0.00 & $\mp 0.07$ & $\mp 0.09$ & 0.11 \\
$\mathrm{Y}$ & \pm 0.07 & \pm 0.04 & \pm 0.04 & \pm 0.01 & 0.09 \\
$\mathrm{Zr}$ & \pm 0.00 & \pm 0.05 & $\mp 0.05$ & \pm 0.00 & 0.07 \\
$\mathrm{Ba}$ & \pm 0.04 & \pm 0.04 & \pm 0.04 & $\mp 0.06$ & 0.09 \\
$\mathrm{La}$ & \pm 0.00 & \pm 0.05 & $\mp 0.05$ & \pm 0.00 & 0.07 \\
$\mathrm{Nd}$ & $\mp 0.06$ & \pm 0.04 & \pm 0.04 & \pm 0.02 & 0.08 \\
$\mathrm{Eu}$ & \pm 0.00 & \pm 0.07 & $\mp 0.05$ & \pm 0.00 & 0.09 \\
\hline & & & $\mathrm{UVES}: \mathrm{I}-53$ & & \\
& $\pm 50 \mathrm{~K}$ & \pm 0.14 & \pm 0.10 & $\pm 0.13 \mathrm{~km} \mathrm{~s}$ & \\
$\mathrm{O}$ & \pm 0.00 & \pm 0.06 & $\mp 0.05$ & \pm 0.00 & 0.08 \\
$\mathrm{Na}$ & $\mp 0.02$ & $\mp 0.01$ & $\mp 0.01$ & \pm 0.01 & 0.03 \\
$\mathrm{Mg}$ & $\mp 0.03$ & \pm 0.00 & $\mp 0.01$ & \pm 0.01 & 0.03 \\
$\mathrm{Al}$ & $\mp 0.03$ & $\mp 0.01$ & $\mp 0.01$ & \pm 0.02 & 0.04 \\
$\mathrm{Si}$ & $\mp 0.05$ & \pm 0.01 & \pm 0.01 & \pm 0.01 & 0.05 \\
$\mathrm{Ca}$ & $\mp 0.01$ & $\mp 0.01$ & $\mp 0.01$ & $\mp 0.01$ & 0.02 \\
$\mathrm{Ti}$ I & \pm 0.04 & $\mp 0.01$ & $\mp 0.02$ & \pm 0.00 & 0.05 \\
$\mathrm{Ti} i_{I I}$ & \pm 0.02 & $\mp 0.01$ & $\mp 0.01$ & $\mp 0.02$ & 0.03 \\
$\mathrm{Fe} \mathrm{I}_{\mathrm{I}}$ & \pm 0.07 & \pm 0.00 & $\mp 0.01$ & $\mp 0.02$ & 0.07 \\
$\mathrm{Fe}$ & $\mp 0.02$ & \pm 0.05 & \pm 0.02 & $\mp 0.02$ & 0.06 \\
$\mathrm{Cu}$ & \pm 0.05 & \pm 0.00 & $\mp 0.04$ & $\mp 0.05$ & 0.08 \\
$\mathrm{Zn}$ & \pm 0.00 & \pm 0.00 & $\mp 0.08$ & $\mp 0.08$ & 0.11 \\
$\mathrm{Y}$ & \pm 0.03 & $\mp 0.01$ & \pm 0.00 & $\mp 0.02$ & 0.04 \\
$\mathrm{Zr}$ & \pm 0.03 & \pm 0.00 & \pm 0.00 & \pm 0.02 & 0.04 \\
$\mathrm{Ba}$ & \pm 0.05 & $\mp 0.01$ & \pm 0.00 & $\mp 0.09$ & 0.10 \\
$\mathrm{La}$ & \pm 0.05 & \pm 0.07 & $\mp 0.03$ & \pm 0.02 & 0.09 \\
$\mathrm{Nd}$ & $\mp 0.08$ & \pm 0.00 & \pm 0.00 & \pm 0.04 & 0.09 \\
$\mathrm{Eu}$ & \pm 0.02 & \pm 0.05 & $\mp 0.08$ & \pm 0.00 & 0.10 \\
\hline & & & & &
\end{tabular}

Notes. ${ }^{(a)}$ For $\mathrm{Fe}_{\mathrm{I}}$ and $\mathrm{Fe}_{\mathrm{II}}$ the $[\mathrm{X} / \mathrm{H}]$ sensitivities are given; for and all remaining elements we list the sensitivities for the abundance ratios [el/Fe]. (b) Quadratic sum of the contributions given by each atmospheric parameter. 
Table 5. Light element abundances.

\begin{tabular}{|c|c|c|c|c|c|c|c|c|c|c|c|c|c|c|}
\hline Star & Source & $\mathrm{Fe}^{a}$ & $\mathrm{C}^{b}$ & $\mathrm{~N}^{c}$ & $\mathrm{O}$ & $\mathrm{Na}_{\text {LTE }}$ & $\mathrm{Mg}$ & $\mathrm{Al}$ & $\mathrm{Si}$ & $\mathrm{Ca}$ & TiI & TiII & ${ }^{12} \mathrm{C} /{ }^{13} \mathrm{C}$ & $s$-rich? \\
\hline \multirow[t]{4}{*}{$\mathrm{I}-12$} & MCD & -1.90 & -0.81 & 0.98 & $\ldots$ & 0.38 & 0.27 & 0.76 & 0.46 & 0.29 & 0.20 & 0.33 & 4.00 & no \\
\hline & AAT & -1.86 & $\ldots$ & $\ldots$ & 0.24 & 0.39 & 0.35 & 0.66 & 0.33 & 0.21 & 0.20 & $\ldots$ & $\ldots$ & no \\
\hline & UVES & -1.85 & $\ldots$ & $\ldots$ & 0.18 & 0.31 & 0.34 & 0.60 & 0.44 & 0.20 & 0.22 & 0.35 & $\ldots$ & no \\
\hline & AVG. & -1.87 & -0.81 & 0.98 & 0.21 & 0.36 & 0.32 & 0.67 & 0.41 & 0.23 & 0.21 & 0.34 & $\ldots$ & no \\
\hline \multirow[t]{3}{*}{$\mathrm{I}-27$} & APO & -1.81 & $\ldots$ & $\ldots$ & 0.55 & 0.32 & 0.50 & 0.49 & 0.58 & 0.32 & 0.27 & 0.41 & $\ldots$ & yes \\
\hline & UVES & -1.63 & $\ldots$ & $\ldots$ & 0.52 & 0.24 & 0.34 & 0.33 & 0.44 & 0.35 & 0.22 & 0.41 & $\ldots$ & yes \\
\hline & AVG. & -1.72 & $\ldots$ & $\ldots$ & 0.54 & 0.28 & 0.42 & 0.41 & 0.51 & 0.34 & 0.25 & 0.41 & $\ldots$ & yes \\
\hline I-36 & LICK & -1.89 & $\ldots$ & $\ldots$ & 0.38 & -0.05 & 0.50 & 0.52 & 0.42 & 0.27 & 0.10 & 0.32 & $\ldots$ & no \\
\hline I-37 & LICK & -1.73 & $\ldots$ & $\ldots$ & 0.37 & 0.25 & 0.18 & 0.51 & 0.54 & 0.23 & 0.11 & 0.26 & $\ldots$ & no \\
\hline I-53 & UVES & -1.74 & $\ldots$ & $\ldots$ & 0.35 & 0.29 & 0.51 & 0.39 & 0.45 & 0.35 & 0.25 & 0.36 & $\ldots$ & yes \\
\hline \multirow[t]{3}{*}{ I-57 } & APO & -1.65 & $\ldots$ & $\ldots$ & 0.59 & 0.33 & 0.34 & 0.10 & 0.53 & 0.42 & 0.20 & 0.30 & $\ldots$ & yes \\
\hline & AAT & -1.62 & $\ldots$ & $\ldots$ & 0.57 & 0.29 & 0.46 & 0.27 & 0.51 & 0.38 & 0.23 & $\ldots$ & $\ldots$ & yes \\
\hline & AVG. & -1.64 & $\ldots$ & $\ldots$ & 0.58 & 0.31 & 0.40 & 0.19 & 0.52 & 0.40 & 0.22 & 0.30 & $\ldots$ & yes \\
\hline I-80 & UVES & -1.70 & -0.40 & 1.33 & -0.10 & 0.65 & 0.49 & 0.84 & 0.41 & 0.35 & 0.19 & 0.28 & $\ldots$ & yes \\
\hline I- 85 & UVES & -1.81 & -0.91 & 1.03 & -0.03 & 0.59 & 0.22 & 0.64 & 0.46 & 0.25 & 0.18 & 0.28 & $\ldots$ & no \\
\hline \multirow[t]{3}{*}{ I-86 } & AAT & -1.80 & $\ldots$ & $\ldots$ & 0.40 & 0.00 & 0.38 & $\ldots$ & 0.47 & 0.34 & $\ldots$ & $\ldots$ & $\ldots$ & no \\
\hline & UVES & -1.84 & $\ldots$ & $\ldots$ & 0.45 & -0.01 & 0.23 & 0.04 & 0.35 & 0.22 & 0.23 & 0.35 & $\ldots$ & no \\
\hline & AVG. & -1.82 & $\ldots$ & $\ldots$ & 0.43 & 0.00 & 0.31 & 0.04 & 0.41 & 0.28 & 0.23 & 0.35 & $\ldots$ & no \\
\hline I-92 & LICK & -1.75 & $\ldots$ & $\ldots$ & 0.52 & -0.05 & 0.32 & $\ldots$ & 0.47 & 0.23 & 0.16 & 0.35 & $\ldots$ & no \\
\hline II-1 & LICK & -1.66 & $\ldots$ & $\ldots$ & 0.48 & 0.11 & 0.47 & 0.44 & 0.54 & 0.37 & 0.21 & 0.43 & $\ldots$ & yes \\
\hline II-31 & LICK & -1.65 & $\ldots$ & $\ldots$ & 0.47 & -0.11 & 0.36 & -0.07 & 0.33 & 0.29 & 0.13 & 0.29 & $\ldots$ & no \\
\hline II-96 & MCD & -1.82 & -0.96 & 0.75 & 0.47 & 0.29 & 0.21 & 0.40 & 0.43 & 0.21 & 0.07 & 0.26 & 4.00 & no \\
\hline II-104 & UVES & -1.76 & -0.60 & 0.53 & 0.37 & -0.20 & 0.33 & -0.06 & 0.43 & 0.25 & 0.20 & 0.35 & $\ldots$ & no \\
\hline \multirow[t]{4}{*}{ III-3 } & MCD & -1.72 & -0.01 & 0.93 & 0.49 & 0.36 & 0.39 & 0.14 & 0.58 & 0.26 & 0.27 & 0.15 & 3.50 & yes \\
\hline & APO & -1.78 & $\ldots$ & $\ldots$ & 0.50 & 0.25 & 0.34 & 0.18 & 0.62 & 0.30 & 0.44 & 0.35 & $\ldots$ & yes \\
\hline & UVES & -1.66 & $\ldots$ & $\ldots$ & 0.25 & 0.31 & 0.34 & 0.20 & 0.42 & 0.34 & 0.33 & 0.38 & $\ldots$ & yes \\
\hline & AVG. & -1.72 & -0.01 & 0.93 & 0.41 & 0.31 & 0.36 & 0.17 & 0.54 & 0.30 & 0.35 & 0.29 & $\ldots$ & yes \\
\hline \multirow[t]{3}{*}{ III-12 } & MCD & -1.69 & -0.26 & 1.13 & 0.40 & 0.52 & 0.43 & 0.55 & 0.33 & 0.33 & 0.17 & 0.28 & 4.00 & yes \\
\hline & AAT & -1.61 & $\ldots$ & $\ldots$ & 0.50 & 0.46 & 0.30 & 0.45 & 0.43 & 0.21 & 0.25 & $\ldots$ & $\ldots$ & yes \\
\hline & AVG. & -1.65 & -0.26 & 1.13 & 0.45 & 0.49 & 0.37 & 0.50 & 0.38 & 0.27 & 0.21 & 0.28 & $\ldots$ & yes \\
\hline \multirow[t]{3}{*}{ III-14 } & LICK & -1.84 & $\ldots$ & $\ldots$ & 0.50 & 0.14 & 0.42 & -0.10 & 0.53 & 0.17 & 0.26 & 0.39 & $\ldots$ & no \\
\hline & AAT & -1.80 & $\ldots$ & $\ldots$ & 0.46 & 0.13 & 0.40 & 0.05 & 0.42 & 0.15 & 0.24 & $\ldots$ & $\ldots$ & no \\
\hline & AVG. & -1.82 & $\ldots$ & $\ldots$ & 0.48 & 0.14 & 0.41 & -0.03 & 0.48 & 0.16 & 0.25 & 0.39 & $\ldots$ & no \\
\hline III-15 & LICK & -1.82 & $\ldots$ & $\ldots$ & 0.11 & 0.54 & 0.48 & 0.63 & 0.62 & 0.33 & 0.24 & 0.40 & $\ldots$ & no \\
\hline III-25 & UVES & -1.92 & $\ldots$ & $\ldots$ & 0.56 & 0.02 & 0.37 & 0.27 & 0.44 & 0.32 & 0.17 & 0.31 & $\ldots$ & no \\
\hline III-33 & UVES & -1.78 & -0.55 & 0.63 & 0.34 & -0.17 & 0.54 & -0.07 & 0.40 & 0.28 & 0.19 & 0.28 & $\ldots$ & no \\
\hline III-35 & UVES & -1.83 & $\ldots$ & $\ldots$ & 0.19 & 0.43 & 0.54 & 0.47 & 0.37 & 0.30 & 0.20 & 0.36 & $\ldots$ & no \\
\hline III-47 & APO & -1.82 & $\ldots$ & $\ldots$ & 0.50 & 0.30 & 0.38 & 0.41 & 0.39 & 0.31 & 0.11 & 0.18 & $\ldots$ & no \\
\hline III-50 & UVES & -1.76 & -0.10 & 1.03 & 0.35 & 0.34 & 0.56 & 0.46 & 0.44 & 0.45 & 0.27 & 0.27 & $\ldots$ & yes \\
\hline \multirow[t]{3}{*}{ III-52 } & LICK & -1.63 & $\ldots$ & $\ldots$ & 0.40 & 0.30 & 0.42 & 0.11 & 0.37 & 0.35 & 0.26 & 0.23 & $\ldots$ & yes \\
\hline & UVES & -1.62 & $\ldots$ & $\ldots$ & 0.49 & 0.26 & 0.44 & 0.27 & 0.48 & 0.42 & 0.38 & 0.44 & $\ldots$ & yes \\
\hline & AVG. & -1.63 & $\ldots$ & $\ldots$ & 0.45 & 0.28 & 0.43 & 0.19 & 0.43 & 0.39 & 0.32 & 0.34 & $\ldots$ & yes \\
\hline III-96 & AAT & -1.86 & $\ldots$ & $\ldots$ & 0.48 & 0.01 & 0.51 & $\ldots$ & 0.30 & 0.28 & 0.25 & $\ldots$ & $\ldots$ & no \\
\hline \multirow[t]{3}{*}{ IV-20 } & AAT & -1.65 & $\ldots$ & $\ldots$ & $\ldots$ & 0.80 & 0.29 & 0.82 & 0.32 & 0.33 & 0.32 & $\ldots$ & $\ldots$ & yes \\
\hline & UVES & -1.63 & -0.61 & 1.33 & -0.05 & 0.73 & 0.44 & 0.74 & 0.47 & 0.40 & 0.28 & 0.36 & $\ldots$ & yes \\
\hline & AVG. & -1.64 & -0.61 & 1.33 & -0.05 & 0.77 & 0.37 & 0.78 & 0.40 & 0.37 & 0.30 & 0.36 & $\ldots$ & yes \\
\hline IV-59 & UVES & -1.77 & $\ldots$ & $\ldots$ & 0.14 & 0.59 & 0.26 & 0.75 & 0.42 & 0.29 & 0.25 & 0.34 & $\ldots$ & no \\
\hline IV-68 & UVES & -1.75 & -0.42 & 0.63 & 0.36 & -0.22 & 0.29 & -0.15 & 0.41 & 0.33 & 0.29 & 0.25 & $\ldots$ & no \\
\hline IV-76 & AAT & -1.63 & $\ldots$ & $\ldots$ & 0.56 & 0.19 & 0.31 & $\ldots$ & 0.44 & 0.22 & 0.14 & $\ldots$ & $\ldots$ & yes \\
\hline IV-88 & AAT & -1.62 & $\ldots$ & $\ldots$ & 0.02 & 0.61 & 0.17 & 0.77 & 0.48 & 0.35 & 0.26 & $\ldots$ & $\ldots$ & yes \\
\hline IV-97 & UVES & -1.94 & -0.50 & 0.30 & 0.40 & -0.02 & 0.46 & 0.07 & 0.44 & 0.24 & 0.26 & 0.42 & $\ldots$ & no \\
\hline \multirow[t]{4}{*}{ IV-102 } & LICK & -1.96 & $\ldots$ & $\ldots$ & 0.44 & 0.41 & 0.45 & 0.19 & $\ldots$ & 0.33 & 0.30 & 0.27 & $\ldots$ & no \\
\hline & $\mathrm{MCD}$ & -1.95 & -0.46 & 0.48 & 0.41 & 0.28 & 0.42 & 0.29 & 0.42 & 0.25 & 0.21 & 0.26 & 3.00 & no \\
\hline & AAT & -2.01 & $\ldots$ & $\ldots$ & 0.44 & 0.40 & 0.52 & $\ldots$ & 0.49 & 0.21 & 0.33 & $\ldots$ & $\ldots$ & no \\
\hline & AVG. & -1.97 & -0.46 & 0.48 & 0.43 & 0.36 & 0.46 & 0.24 & 0.46 & 0.26 & 0.28 & 0.27 & $\ldots$ & no \\
\hline $\mathrm{C}$ & $\mathrm{MCD}$ & -1.69 & -0.41 & 1.10 & 0.25 & 0.68 & 0.43 & 0.58 & 0.38 & 0.37 & 0.38 & 0.20 & 5.00 & yes \\
\hline C513 & APO & -1.86 & $\ldots$ & $\ldots$ & 0.40 & 0.03 & 0.57 & $\ldots$ & 0.40 & 0.22 & 0.14 & 0.34 & $\ldots$ & no \\
\hline $\mathrm{V}-2$ & LICK & -1.57 & $\ldots$ & $\ldots$ & 0.15 & 0.56 & 0.21 & 0.79 & 0.40 & 0.45 & 0.30 & 0.30 & $\ldots$ & yes \\
\hline
\end{tabular}

Notes. ${ }^{(a)}$ For this column only, metallicities $[\mathrm{Fe} / \mathrm{H}]$ are given. ${ }^{(b)}$ For this and all remaining abundances, [el/Fe] values are given.

${ }^{(c)}$ For the stars II-104, III-33, IV-68, IV-97, and IV-102, we were able to measure only upper limits for the nitrogen abundance. 
Table 6. Heavy element abundances.

\begin{tabular}{|c|c|c|c|c|c|c|c|c|c|c|c|}
\hline Star & Source & $\mathrm{Fe}^{a}$ & $\mathrm{Cu}^{b}$ & $\mathrm{Zn}$ & Y & $\mathrm{Zr}$ & $\mathrm{Ba}$ & $\mathrm{La}$ & $\mathrm{Nd}$ & $\mathrm{Eu}$ & $s$-rich? \\
\hline \multirow{4}{*}{ I-12 } & MCD & -1.90 & -0.93 & 0.13 & -0.20 & -0.05 & 0.03 & -0.12 & -0.03 & $\ldots$ & no \\
\hline & AAT & -1.86 & -0.93 & $\ldots$ & $\ldots$ & $\ldots$ & -0.20 & 0.03 & $\ldots$ & $\ldots$ & no \\
\hline & UVES & -1.85 & -1.00 & 0.15 & -0.07 & -0.03 & 0.01 & -0.07 & 0.03 & 0.43 & no \\
\hline & AVG. & -1.87 & -0.95 & 0.14 & -0.14 & -0.04 & -0.05 & -0.05 & 0.00 & 0.43 & no \\
\hline \multirow[t]{3}{*}{ I-27 } & APO & -1.81 & -0.90 & 0.08 & 0.34 & 0.58 & 0.20 & $\ldots$ & 0.48 & . & yes \\
\hline & UVES & -1.63 & -0.78 & 0.10 & 0.40 & 0.46 & 0.52 & 0.47 & 0.44 & 0.45 & yes \\
\hline & AVG. & -1.72 & -0.84 & 0.09 & 0.37 & 0.52 & 0.36 & 0.47 & 0.46 & 0.45 & yes \\
\hline I-36 & LICK & -1.89 & -1.38 & $\ldots$ & -0.15 & $\ldots$ & -0.03 & -0.05 & 0.04 & $\ldots$ & no \\
\hline I-37 & LICK & -1.73 & -0.90 & $\ldots$ & -0.13 & 0.43 & 0.02 & 0.03 & -0.06 & 0.45 & no \\
\hline I-53 & UVES & -1.74 & -0.75 & 0.15 & 0.26 & 0.35 & 0.54 & 0.29 & 0.31 & 0.41 & yes \\
\hline \multirow[t]{3}{*}{ I-57 } & APO & -1.65 & -0.75 & 0.25 & 0.45 & 0.60 & 0.22 & 0.41 & 0.42 & 0.47 & yes \\
\hline & AAT & -1.62 & -0.65 & $\ldots$ & $\ldots$ & $\ldots$ & 0.20 & 0.27 & $\ldots$ & $\ldots$ & yes \\
\hline & AVG. & -1.64 & -0.70 & 0.25 & 0.45 & 0.60 & 0.21 & 0.34 & 0.42 & 0.47 & yes \\
\hline I- 80 & UVES & -1.70 & -0.85 & 0.15 & 0.24 & 0.38 & 0.50 & 0.22 & 0.18 & 0.30 & yes \\
\hline I- 85 & UVES & -1.81 & -1.10 & 0.10 & -0.27 & -0.03 & 0.14 & 0.03 & -0.13 & 0.44 & no \\
\hline \multirow[t]{3}{*}{ I-86 } & AAT & -1.80 & -0.98 & 0.05 & $\ldots$ & $\ldots$ & 0.12 & -0.05 & $\ldots$ & & no \\
\hline & UVES & -1.84 & -1.00 & $\ldots$ & -0.18 & 0.00 & -0.10 & 0.05 & 0.08 & 0.52 & no \\
\hline & AVG. & -1.82 & -0.99 & 0.05 & -0.18 & 0.00 & 0.01 & 0.00 & 0.08 & 0.52 & no \\
\hline I-92 & LICK & -1.75 & -0.78 & $\ldots$ & 0.07 & $\ldots$ & -0.11 & -0.01 & -0.01 & 0.43 & no \\
\hline II-1 & LICK & -1.66 & -0.93 & $\ldots$ & 0.20 & 0.55 & 0.02 & 0.32 & 0.38 & 0.40 & yes \\
\hline II-31 & LICK & -1.65 & -0.88 & $\ldots$ & 0.05 & 0.10 & -0.23 & 0.05 & 0.14 & 0.52 & no \\
\hline II-96 & MCD & -1.82 & -0.90 & -0.02 & -0.22 & 0.10 & -0.18 & 0.09 & 0.05 & 0.56 & no \\
\hline II-104 & UVES & -1.76 & -1.00 & 0.05 & -0.09 & -0.05 & 0.14 & -0.01 & 0.05 & 0.50 & no \\
\hline \multirow[t]{4}{*}{ III-3 } & MCD & -1.72 & -0.78 & 0.13 & 0.56 & 0.40 & 0.24 & 0.23 & 0.40 & 0.45 & yes \\
\hline & APO & -1.78 & -0.67 & 0.33 & 0.42 & 0.44 & 0.31 & 0.33 & 0.49 & 0.35 & yes \\
\hline & UVES & -1.66 & -0.63 & 0.15 & 0.58 & 0.36 & 0.33 & 0.16 & 0.35 & 0.45 & yes \\
\hline & AVG. & -1.72 & -0.69 & 0.20 & 0.52 & 0.40 & 0.29 & 0.24 & 0.41 & 0.42 & yes \\
\hline \multirow[t]{3}{*}{ III-12 } & $\mathrm{MCD}$ & -1.69 & -0.72 & 0.23 & 0.37 & 0.54 & 0.29 & 0.39 & 0.50 & 0.55 & yes \\
\hline & AAT & -1.61 & -0.68 & $\ldots$ & $\ldots$ & $\ldots$ & 0.31 & 0.48 & $\ldots$ & $\ldots$ & yes \\
\hline & AVG. & -1.65 & -0.70 & 0.23 & 0.37 & 0.54 & 0.30 & 0.44 & 0.50 & 0.55 & yes \\
\hline \multirow[t]{3}{*}{ III-14 } & LICK & -1.84 & -0.70 & $\ldots$ & 0.10 & 0.10 & 0.04 & 0.12 & 0.21 & 0.58 & no \\
\hline & AAT & -1.80 & -0.95 & $\ldots$ & $\ldots$ & $\ldots$ & -0.28 & -0.03 & $\ldots$ & . & no \\
\hline & AVG. & -1.82 & -0.83 & $\ldots$ & 0.10 & 0.10 & -0.12 & 0.05 & 0.21 & 0.58 & no \\
\hline III-15 & LICK & -1.82 & -0.80 & $\ldots$ & 0.09 & -0.05 & -0.05 & 0.05 & 0.16 & 0.46 & no \\
\hline III-25 & UVES & -1.92 & -1.15 & 0.10 & -0.23 & 0.15 & -0.05 & 0.13 & 0.11 & 0.50 & no \\
\hline III-33 & UVES & -1.78 & -0.95 & 0.05 & -0.21 & 0.00 & -0.11 & -0.04 & 0.01 & 0.40 & no \\
\hline III-35 & UVES & -1.83 & -0.95 & 0.20 & -0.06 & -0.05 & 0.10 & 0.01 & 0.01 & 0.50 & no \\
\hline III-47 & APO & -1.82 & -0.78 & -0.02 & 0.16 & 0.50 & 0.01 & $\ldots$ & 0.03 & 0.50 & no \\
\hline III-50 & UVES & -1.76 & -0.90 & 0.15 & 0.20 & 0.30 & 0.27 & 0.20 & 0.16 & 0.35 & yes \\
\hline \multirow[t]{3}{*}{ III-52 } & LICK & -1.63 & -0.84 & $\ldots$ & 0.32 & 0.36 & 0.38 & 0.24 & 0.30 & 0.40 & yes \\
\hline & UVES & -1.62 & -0.73 & 0.18 & 0.36 & 0.40 & 0.46 & 0.29 & 0.49 & 0.43 & yes \\
\hline & AVG. & -1.63 & -0.78 & 0.18 & 0.34 & 0.38 & 0.42 & 0.27 & 0.40 & 0.42 & yes \\
\hline III-96 & AAT & -1.86 & -0.90 & $\ldots$ & $\ldots$ & $\ldots$ & -0.21 & -0.03 & $\ldots$ & $\ldots$ & no \\
\hline \multirow[t]{3}{*}{ IV-20 } & AAT & -1.65 & -0.78 & $\ldots$ & $\ldots$ & $\ldots$ & 0.30 & 0.49 & $\ldots$ & $\ldots$ & yes \\
\hline & UVES & -1.63 & -0.78 & 0.20 & 0.32 & 0.36 & 0.40 & 0.29 & 0.40 & 0.34 & yes \\
\hline & AVG. & -1.64 & -0.78 & 0.20 & 0.32 & 0.36 & 0.35 & 0.39 & 0.40 & 0.34 & yes \\
\hline IV-59 & UVES & -1.77 & -0.90 & 0.00 & -0.14 & -0.04 & -0.10 & -0.02 & -0.02 & 0.46 & no \\
\hline IV-68 & UVES & -1.75 & -1.05 & 0.00 & -0.29 & -0.04 & 0.16 & 0.10 & -0.01 & 0.53 & no \\
\hline IV-76 & AAT & -1.63 & -0.88 & $\ldots$ & $\ldots$ & $\ldots$ & 0.19 & 0.35 & $\ldots$ & $\ldots$ & yes \\
\hline IV-88 & AAT & -1.62 & -0.75 & $\ldots$ & $\ldots$ & $\ldots$ & 0.39 & 0.47 & $\ldots$ & $\ldots$ & yes \\
\hline IV-97 & UVES & -1.94 & -0.83 & 0.30 & -0.01 & 0.00 & 0.00 & -0.13 & 0.02 & 0.48 & no \\
\hline \multirow[t]{4}{*}{ IV-102 } & LICK & -1.96 & -1.18 & $\ldots$ & 0.01 & -0.10 & -0.06 & -0.13 & 0.07 & $\ldots$ & no \\
\hline & MCD & -1.95 & -0.95 & 0.23 & 0.06 & 0.00 & -0.26 & -0.07 & 0.05 & 0.52 & no \\
\hline & AAT & -2.01 & -0.73 & $\ldots$ & $\ldots$ & $\ldots$ & -0.14 & -0.07 & $\ldots$ & $\ldots$ & no \\
\hline & AVG. & -1.97 & -0.95 & 0.23 & 0.04 & -0.05 & -0.15 & -0.09 & 0.06 & 0.52 & no \\
\hline C & MCD & -1.69 & -0.82 & 0.03 & 0.43 & 0.30 & 0.26 & 0.31 & 0.34 & 0.52 & yes \\
\hline C513 & APO & -1.86 & -0.82 & 0.25 & -0.07 & 0.15 & -0.26 & 0.06 & 0.22 & 0.52 & no \\
\hline $\mathrm{V}-2$ & LICK & -1.57 & -0.63 & $\ldots$ & 0.26 & 0.30 & 0.22 & 0.29 & 0.51 & $\ldots$ & yes \\
\hline
\end{tabular}

Notes. ${ }^{(a)}$ For this column only, metallicities $[\mathrm{Fe} / \mathrm{H}]$ are given. ${ }^{(b)}$ For this and all remaining abundances, [el/Fe] values are given. 